\title{
Thermisch induzierte Gestaltinstabilität von Vielfachschichten
}

\author{
Dissertation \\ zur Erlangung des Doktorgrades \\ der Mathematisch-Naturwissenschaftlichen Fakultät \\ der Georg-August-Universität zu Göttingen
}

vorgelegt von

Peter Troche

aus Köln

Göttingen 2000 


\section{7}

Referent: Prof. Dr. H.C. Freyhardt

Koreferent: Prof. Dr. G. Schmahl

Tag der mündlichen Prüfung: 20. Juni 2000 


\section{Inhaltsverzeichnis}

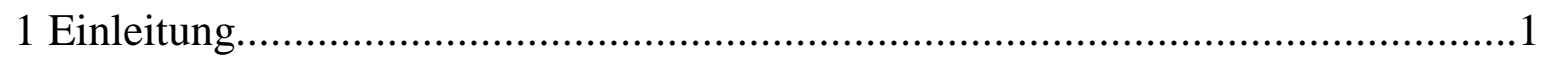

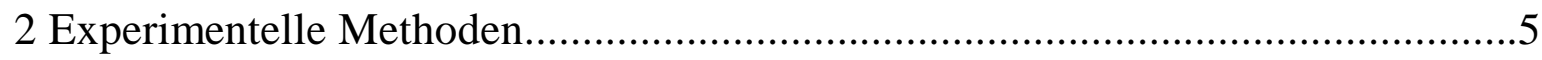

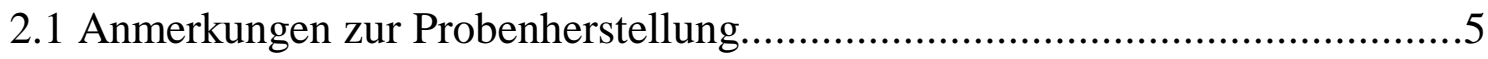

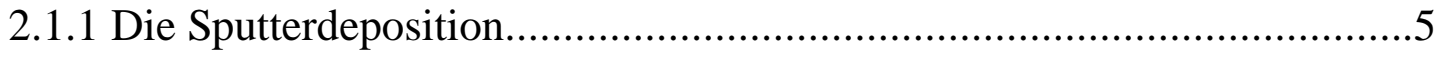

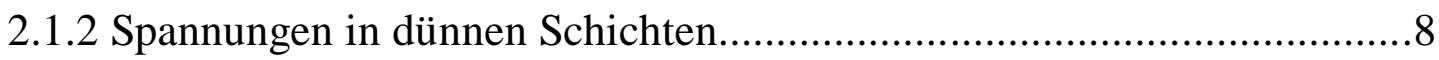

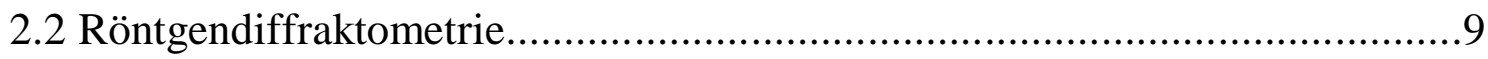

2.2.1 Messungen im 2-Kreis-Diffraktometer.................................................

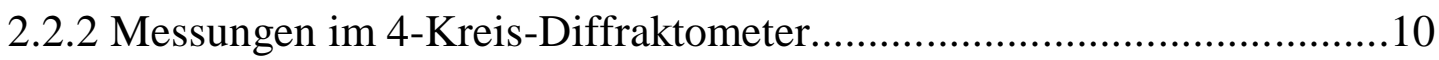

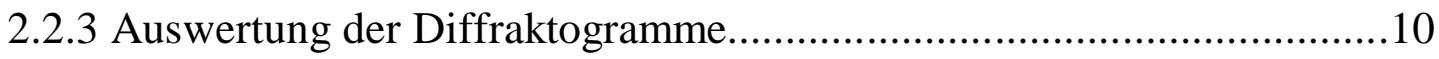

Gitterkonstanten und Korngrößen …....................................................10

Periodenlänge und Grenzflächenrauigkeit von Vielfachschichten..............11

Das Stufenmodell und seine Erweiterung auf Dickenfluktuationen............12

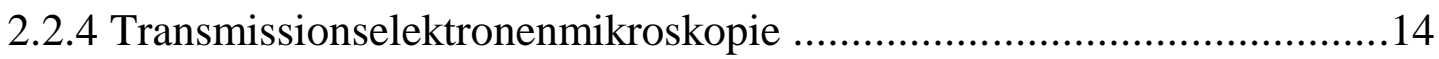

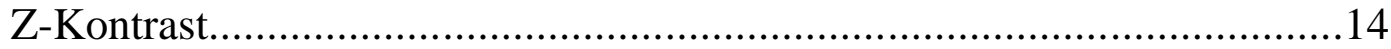

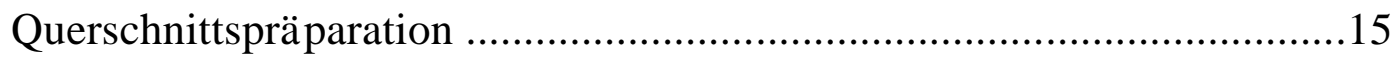

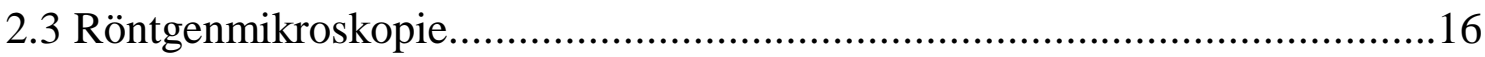

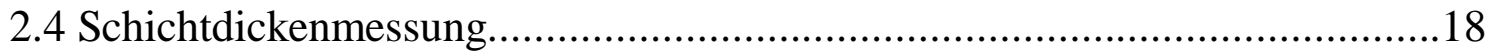

3 Mikrostrukturuntersuchungen an Vielfachschichten.......................................19

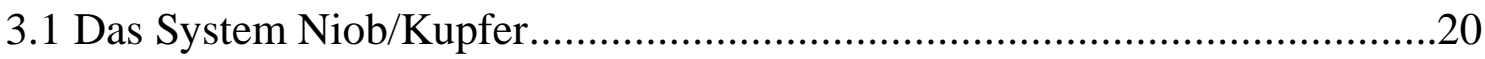

3.2 Die Systeme Eisen/Silber und Eisen/Gold................................................23

3.3 Vergleichssysteme Kupfer/Kobalt, Kohlenstoff/Kobalt...............................32

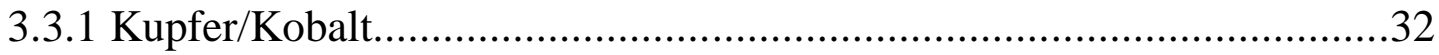

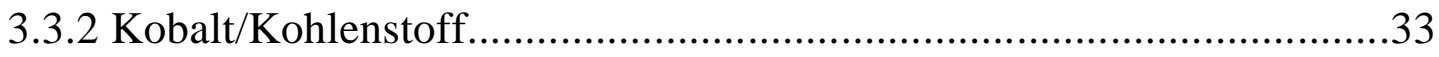

3.4 Übersicht über die wie-hergestellten Proben.................................................35

4 Die Mikrostruktur während und nach der Auslagerung .......................................36

4.1 Desintegration in den Systemen Niob/Kupfer, Eisen/Gold und Eisen/Silber.36

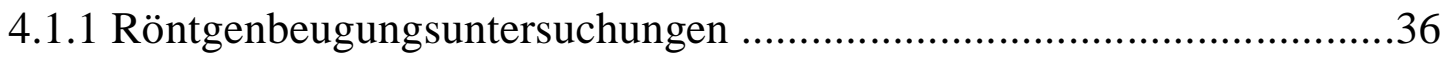

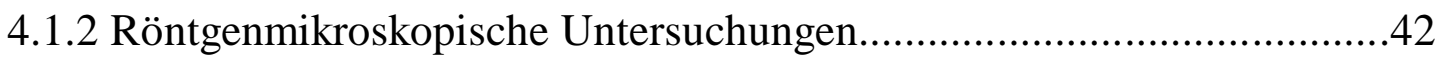

4.1.3 Transmissionselektronenmikroskopische Untersuchungen....................45

4.2 Desintegration in den Systemen Kobalt/Kupfer und Kobalt/Kohlenstoff......51

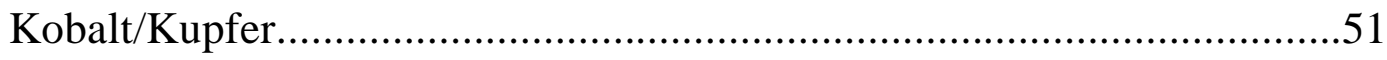

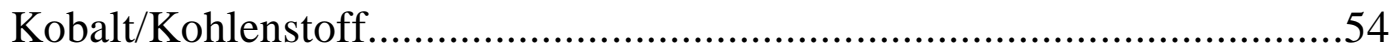

4.3 Vergleich der Mikrostruktur nach der Auslagerung...................................55

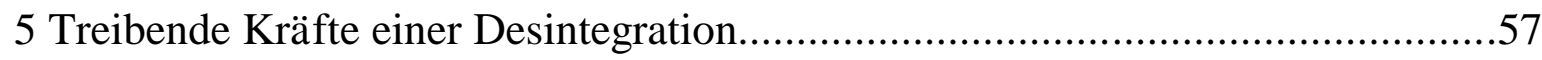

5.1 Cahn-Hilliard-Simulationen und die Desintegration....................................58

5.2 Kristallisation der Matrixschicht und die Desintegration............................59

5.3 Korngrenzkriechen und die Desintegration...........................................60 
5.4 Rauigkeiten der Grenzfläche und die Desintegration..................................60

5.5 Mechanische Grenzflächenspannungen und die Desintegration.....................65

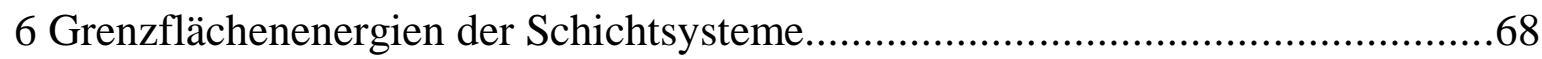

6.1 Der chemische Anteil der Grenzflächenenergie..........................................68

6.2 Der Strukturelle Anteil der Grenzflächenenergie......................................69

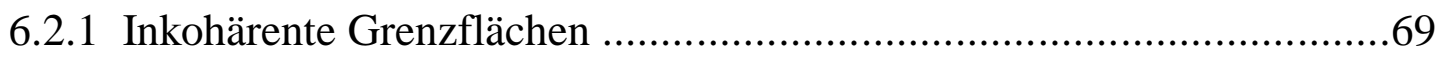

6.2.2 Kohärente und semikohärente Grenzflächen ....................................71

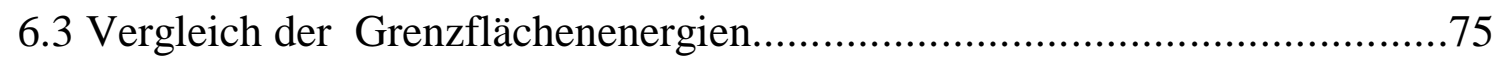

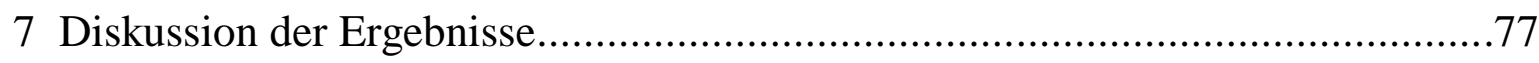

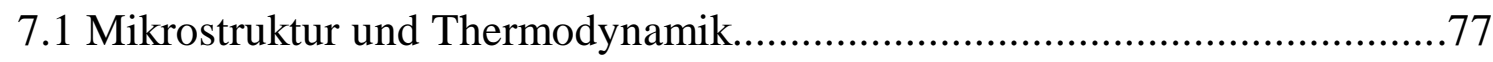

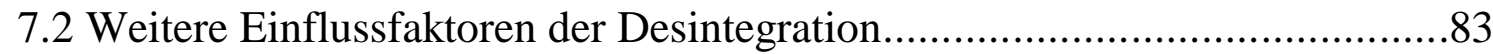

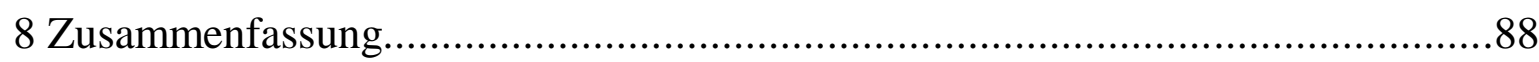

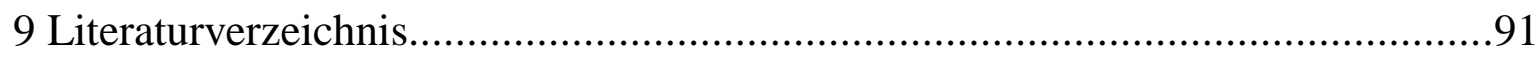

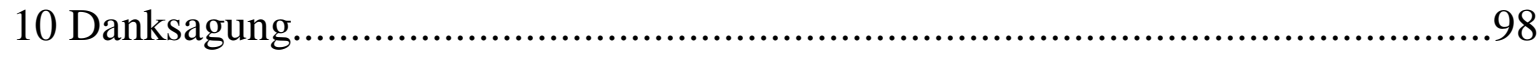




\section{Einleitung}

Vielfachschichten, in denen die Lagendicken der einzelnen Komponenten nur wenige Nanometer betragen, werden in modernen integrierten Geräten in vielfältiger Weise eingesetzt. Um ihren zuverlässigen Einsatz über eine lange Zeit sicherzustellen, ist die Untersuchung von Grenzflächenreaktionen in solchen Systemen von großem Interesse. So können in Vielfachschichtsystemen aus mischenden Komponenten die Bildung von löslichkeitserweiterten Randphasen oder amorphen Phasen durch eine Festkörperreaktion beobachtet werden [Clemens, 1984], [Samwer et al., 1988], [Hartung, 1996].

Von zunehmender Bedeutung sind aber auch Schichtpakete aus nicht mischenden Komponenten, bei denen die Qualität der Grenzflächen, d.h. die Rauigkeit und die thermische Stabilität der Lagenstruktur im Vordergrund steht. So basieren z.B. Röntgenspiegel auf der Beugung von Röntgenstrahlen an der periodischen Lagenstruktur von Schichtpaketen [Spiller, 1987], [Lovesey, 1996]. In GMR-Sensoren wird die besondere magnetische Ordnung in den Lagenstrukturen zur Detektion sehr kleiner magnetischer Felder eingesetzt [Clemens, 1997]. Gerade das letzte Beispiel macht deutlich, dass solche Schichtsysteme bei ihrem Einsatz thermischen Belastungen ausgesetzt sein können (z.B. bei Leseköpfen für Festplatten oder Drehzahlmessern an Motoren mit Abwärme).

Schichtpakete aus nicht mischenden Komponenten zeigen aber zum Teil bei niedrigen Temperaturen einen Zerfall der einzelnen Lagen in getrennte Partikel [Troche et al., 1999], [Herweg, 1999], d.h. einen Prozess, bei dem die periodische Struktur der Vielfachschicht verloren geht. Die resultierende Partikelverteilung ist dann nicht notwendigerweise statistisch. Es können neue, geordnete Strukturen entstehen. Ein Beispiel für eine solche Ordnung ist die bevorzugte Bildung von Eisenpartikeln an Korngrenzen, wie sie z.B. in Eisen/Gold-Schichtpaketen beobachtet wurde [Troche et al., 2000].

Die Arbeiten, die im Rahmen dieser Dissertation an Vielfachschichten nicht mischender Metalle durchgeführt wurden, sollen zum Verständnis der Stabilität von Schichtsystemen mit Periodenlängen von nur wenigen Nanometern während einer Wärmebehandlung beitragen. Zum anderen sollen aber auch Möglichkeiten zur Selbstorganisation von metallischen Nanopartikeln in einer metallischen Matrix un- 
tersucht werden. Auf diese Weise ist die Herstellung von geordneten magnetischen Strukturen zur Datenspeicherung oder die Erzeugung von Quantenstrukturen denkbar. Beiden Fragestellungen ist das Verständnis einer Gestaltinstabilität solcher Schichtsysteme gemeinsam. Besondere Aufmerksamkeit soll dabei vor allem der Mikrostrukturveränderung bei relativ niedrigen Temperaturen von unter $600^{\circ} \mathrm{C}$ geschenkt werden. Die Beantwortung der Frage nach den treibenden Kräften für eine solche Desintegration der Lagenstruktur hin zu einer Mikrostruktur, in der Nanopartikel in einer einheitlichen Matrix vorliegen, ermöglicht die Auswahl von Systemen, die bei Bedarf bis zu hohen Temperaturen stabil sind. Auf der anderen Seite erlaubt dieses Wissen auch, die Mikrostruktur der Systeme im Zustand nach der Deposition so anzupassen, dass eine gezielte Desintegration des Systems in eine gewünschte Struktur stattfindet.

Einen wesentlichen Aspekt in einem Schichtpaket stellt die Untersuchung der Grenzflächen dar. Ihren Einfluss auf die Eigenschaften des Schichtpakets wird dominant, wenn die Periodenlänge nur wenige Nanometer beträgt. Die Grenzflächenenergie beinhaltet einen chemischen und einen strukturellen Anteil. Der chemische Beitrag wird direkt von den beteiligten Metallen und deren Wechselwirkungsenergie bestimmt. Um so größer die repulsive Wechselwirkung, desto größer ist der chemische Anteil der Grenzflächenenergie. Der strukturelle Anteil wird vom Typus der Grenzfläche, der atomaren Anordnung und den dabei auftretenden Fehlpassungsspannungen bestimmt. Eine kohärente Grenzfläche zwischen Materialien ähnlicher Struktur und Gitterkonstante kann einen fast verschwindenden strukturellen Anteil haben. Werden die Spannungen teilweise durch Versetzungen abgebaut, spricht man von semikohärenten Grenzflächen. Ähnelt die Struktur der Grenzfläche einer Großwinkelkorngrenze, bezeichnet man sie als inkohärent [Haasen K3, 1994]. Je nach Typ der Grenzfläche kann der strukturelle Beitrag also verschwinden oder einige $10 \mathrm{~J} / \mathrm{m}^{2}$ betragen [Borchers et al., 2000], [Borchers, Troche et al., 2000].

Neben den energetischen Unterschieden der verschiedenen Arten von Grenzflächen, sind auch mikrostrukturelle Besonderheiten der Grenzflächen zu beachten, die durch Prozesse während des Wachstums des Films entstehen. Inselwachstum bzw. sehr kleine Korngrößen können zu lokalen Rauigkeiten führen. Präparationsbedingte Faktoren wie Abschattungseffekte, aber auch eine Veränderung der lokalen Grenzflächenenergie durch Korngrenzen können zusätzlich auch langwelligere Störungen 
der Grenzflächen induzieren.

Variationen der lokalen Krümmungen der Grenzfläche bzw. Gradienten in der Grenzflächenenergie sind aber treibende Kräfte für eine Diffusion parallel zu den Grenzflächen und sind damit ursächlich für den Zerfall der Schichtstruktur verantwortlich [Cline, 1971], [van Suchtelen, 1977], [Josell, 1999].

Um die Einflussfaktoren auf die thermische Stabilität von Schichtpaketen, d.h. deren Gestaltinstabilität, die im folgenden auch als Desintegration bezeichnet werden soll, separieren zu können, wurden gezielt verschiedene Schichtsysteme untersucht und die Ergebnisse miteinander verglichen. Es handelt sich dabei um die Systeme Eisen/Gold, Eisen/Silber, Niob/Kupfer, Kobalt/Kupfer und Kobalt/Kohlenstoff. Von diesen Systemen haben die Systeme Eisen/Silber und Eisen/Gold sehr ähnliche strukturelle Eigenschaften, sie unterscheiden sich aber stark in ihrer Randlöslichkeit. Andererseits haben die Systeme Eisen/Silber und Kobalt/Kupfer keine nennenswerte Randlöslichkeit, aber trotz kohärenter Grenzflächen sehr unterschiedliche Fehlpassungen. Demzufolge unterscheiden sich die Systeme stark in ihrem strukturellen Anteil der Grenzflächenenergie. Das System Kobalt/Kohlenstoff ist insofern ein Grenzfall, da aufgrund der amorphen Struktur des Kohlenstoff die Grenzflächenenergie allein durch den chemischen Beitrag bestimmt ist. Bei allen Untersuchungen wurden Schichtpakete mit unterschiedlichen Dickenverhältnissen der Komponenten abgeschieden.

Entsprechend wird in den Kapiteln 3 und 4 die Mikrostruktur der Schichtsysteme vor, während und nach der thermischen Behandlung dargestellt. Besonders soll dabei die Korrelation zwischen der Mikrostruktur der unbehandelten Schichten und den Orten bevorzugter Partikelbildung betrachtet werden. Desweiteren werden Experimente zur Bestimmung der Einsatztemperatur bzw. der Kinetik des Desintegrationsprozesses vorgestellt. Im Kapitel 5 werden die möglichen Mechanismen während einer Desintegration betrachtet. Dabei wird besonderen Wert auf solche Vorgänge gelegt, die auf dem Einfluss der Grenzflächen basieren, d.h. etwa einer Variationen der Grenzflächenkrümmung und der mechanischen Spannungen entlang der Grenzfläche. Kapitel 6 führt in die Abschätzung der Energien der verschiedenen Arten von Grenzflächen ein. Basierend auf die Unterscheidung zwischen chemischen und strukturellen Beiträgen, wird zwischen kohärenten, semikohärenten und 
inkohärenten Grenzflächen unterschieden. Schließlich werden in Kapitel 7 die experimentellen Ergebnisse der Kapitel 3 und 4 mit den theoretischen Modellen aus Kapitel 5 und den Energiebetrachtungen zu den Grenzflächen aus Kapitel 6 verglichen und die treibenden Kräfte für die beobachtete Desintegration diskutiert. 


\section{Experimentelle Methoden}

In diesem Kapitel wird zunächst die Sputterdeposition und die Besonderheit der so hergestellten Filme bezüglich innerer Spannungen dargestellt. Danach werden die im Rahmen dieser Arbeit verwendeten Charakterisierungsmethoden vorgestellt.

\subsection{Anmerkungen zur Probenherstellung}

Beim Studium der Literatur über die Eigenschaften dünner Schichten wird schnell klar, dass spezielle Eigenschaften dünner Schichten oft stark von der Herstellungsmethode abhängen. Das hängt im wesentlichen damit zusammen, dass die Energie der auf das Substrat auftreffenden Atome je nach Depositionsverfahren sehr unterschiedlich ist. Die geringste kinetische Energie besitzen die Atome, die mittels Verdampfungsverfahren hergestellt werden. Typische kinetische Energien liegen bei einigen meV [Frey, 1987]. Etwas höher ist die Energie beim Triodensputtern, diejenige Methode, mit der die Schichten, die in dieser Arbeit diskutiert werden, hergestellt wurden. Hier liegt die kinetische Energie etwa bei 1 eV [Frey, 1987]. Beim Ionenstrahlsputtern wird mit einem Ionenstrahl direkt auf ein Target geschossen und das Substrat in die Wolke abgestäubter Atome gehalten. Hier liegt die kinetische Energie höher als beim Triodensputtern, etwa bei 1-10 eV [Frey, 1987]. Sehr hohe kinetische Energien von etwa 10-100 eV [Störmer, 1998] haben die Atome und Ionen, die mittels Laserablation hergestellt werden.

Es ist unmittelbar einsichtig, dass sich die auf diese Weise hergestellten Filme stark unterscheiden. Höhere Energien bedeuten dichtere Filme und größere Durchmischung, wogegen aufgedampfte Filme oft ein sehr großes freies Volumen und eine geringe Durchmischung aufweisen.

\subsubsection{Die Sputterdeposition}

Beim Triodensputtern liegt die Energie der deponierten Atome nur wenig über der kinetischen Energie thermalisierter Atome. Das hängt im wesentlichen mit zwei Ursachen zusammen. Zum einen ist der Druck während der Deposition mit etwa $10^{-2}$ mbar relativ hoch. Zum anderen ist der Abstand zwischen Substrat und Quelle im Vergleich zur mittleren freien Weglänge bei diesem Druck recht groß. Die mitt- 


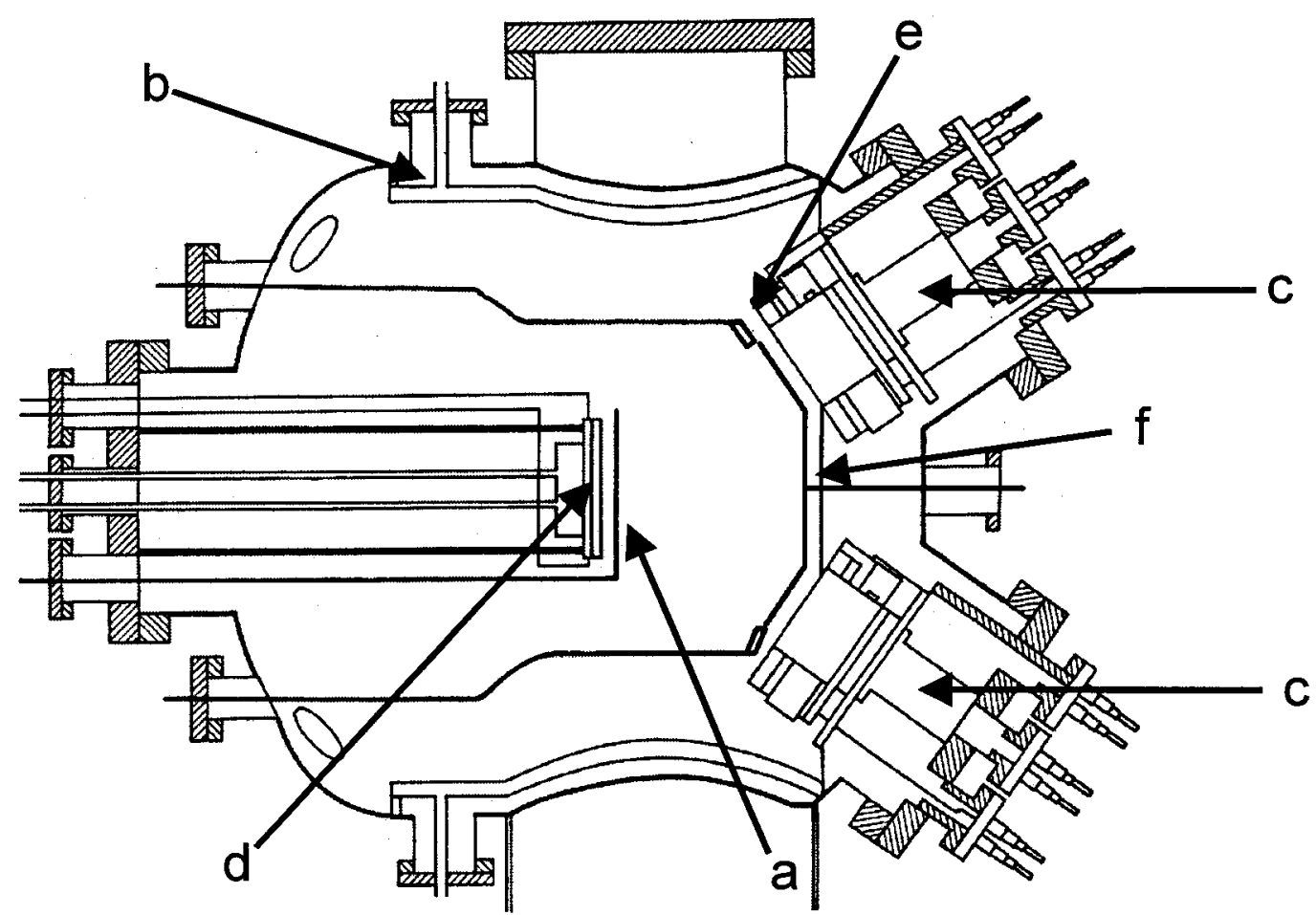

Abbildung 2.1: Schnitt durch die Sputteranlage. (a) Substratblende, (b) Kühlschild zur Verbesserung des Vakuums während der Deposition, (c) Trioden-MagnetronSputterquellen, (d) heizbarer Substrathalter mit rückseitig montiertem Kühlblock, (e) Ratenmonitore, (f) Drehblende zum Verschluss inaktiver Quellen.

lere freie Weglänge beträgt bei einem Druck von $10^{-2}$ mbar nur $3 \mathrm{~cm}$. Das bedeutet, dass die auf dem $23 \mathrm{~cm}$ von der Quelle entfernten Substrat auftreffenden Atome den größten Teil ihrer kinetischen Energie bis dahin verloren haben. Trotzdem besitzen sie eine klare Vorzugsrichtung und gleichen nicht einer vollständig thermalisierten Gaswolke. Dieses Phänomen wird von Drüsedau et al. [Drüsedau et al., 1999] durch eine lokale Verringerung des Drucks im und um das Plasma erklärt.

Die verwendete Sputteranlage ist eine Ultrahochvakuum-Anlage, die bei einem Restgasdruck von besser als $10^{-8}$ mbar betrieben wird (Abbildung 2.1). Um dieses Vakuum zu erreichen, ist sowohl eine Turbomolekularpumpe, als auch eine Kryopumpe installiert. Zusätzlich wird während der Deposition ein in die Vakuumkammer eingebautes Kühlschild mit flüssigem Stickstoff befüllt, an dem hauptsächlich Wasserdampf kondensiert. Ein weiteres Verfahren zur Reduktion des Restgasdrucks besteht darin, einige Stunden vor der eigentlichen Deposition vorzusputtern. Dabei wird der Wasserdampf in Wasserstoff und Sauerstoff gespalten, mit dem dann das gesputterte Metall oxidiert. Ein zweiter Effekt ist die Belegung der Kammerwände 
mit einer frischen Metallschicht, die die Adsorption der ursprünglichen Wand überdeckt und ihrerseits reaktiv ist, um Sauerstoff zu binden. Nach all diesen Maßnahmen erreicht der Sauerstoffpartialdruck ein Niveau von weniger als $10^{-9} \mathrm{mbar}$. Das ist ausreichend, um die Oxidation der Metall-Metall-Grenzflächen der Vielfachschichten während der Deposition zu verhindern.

Die verwendeten Triodenmagnetron-Sputterquellen werden detailiert bei [Hoffmann, 1991] und [Herweg, 1999] dargestellt. In die Sputteranlage können bis zu drei Sputterquellen gleichzeitig eingebaut werden, die unabhängig voneinander betrieben werden können. Sie sind auf einen gemeinsamen Fokus gerichtet, an dem sich der Substrathalter befindet, und um je $90^{\circ}$ gegeneinander versetzt. Ihre Verkippung aus der Längsachse an der Anlage heraus beträgt $35^{\circ}$. Zur Deposition von Vielfachschichten ist vor den Quellen eine Drehblende montiert, die nur die jeweils aktive Quelle öffnet. Sowohl die Quellenblende, als auch die Quellen selbst werden dabei von einem Computerprogramm gesteuert, das für gleich bleibende Schichtdicken und, durch die Schließung der inaktiven Quellen, für Grenzflächen mit extrem geringen Durchmischungen sorgt. Der Substrathalter ist durch einen integrierten Heizer auf Temperaturen bis $650^{\circ} \mathrm{C}$ heizbar und durch Einsatz eines rückseitig anpressbaren Kühlblocks auf bis $\mathrm{zu}-100^{\circ} \mathrm{C}$ mit flüssigem Stickstoff kühlbar. Auf dem Substrathalter können bis zu 30 Substrate in zwei Reihen montiert werden. Je nach Anordnung der Substrate entsteht so ein Gradient in der Aufwachsrate entlang der Reihen. Liegt die Quelle dabei in einer Ebene mit der Mittelachse und der Substratreihe, so ist die maximale Aufwachsrate am quellenseitigen Ende der Reihe etwa $50 \%$ größer als auf der quellenabgewandten Seite. Ist dagegen eine um $90^{\circ}$ dazu verdrehte Quelle in Betrieb, so ist innerhalb der Reihen nahezu kein Gradient vorhanden, sondern nur von einer Reihe zur anderen. Auf diese Weise ist es möglich, reihenweise gleiche Proben herzustellen, und dabei nur die Dicke eines Metalls zu variieren, während die Lagendicke des anderen Metalls konstant bleibt, oder aber die Lagendicken beider Metalle gegenläufig zu verändern. Die Aufwachsrate liegt abhängig von dem gesputterten Material und von der Sputterleistung bei Werten zwischen $0,3 \AA /$ s Kohlenstoff bei $300 \mathrm{~W}$ Sputterleistung und und $7 \AA$ /s Gold bei $300 \mathrm{~W}$. Die maximale Sputterleistung beträgt $550 \mathrm{~W}$. Die Aufwachsrate hängt dabei fast linear von der Sputterleistung ab. Genauere Daten zu den Aufwachsraten und dieser Sputteranlage im Allgemeinen finden sich bei [Hoffmann, 1991]. 
Die im folgenden untersuchten Vielfachschichten sind, wenn es nicht ausdrücklich anders genannt ist, bei Raumtemperatur deponiert worden. Das heißt, dass der Substrathalter während der Deposition nur mit Wasser gekühlt wurde. Durch die Nähe zum Plasma werden die Substrate natürlich von diesem erwärmt. Messungen ergaben, dass die Oberflächen der Substrate bzw. aufwachsenden Filme eine Temperatur von etwa $100^{\circ} \mathrm{C}$ haben, die schon innerhalb der ersten Minuten der Deposition erreicht wird.

\subsubsection{Spannungen in dünnen Schichten}

Dünne Schichten weisen häufig herstellungsbedingte Spannungen auf. Die Ursachen dafür können Unterschiede im thermischen Ausdehnungsvermögen von Substrat und Film, aber auch Defekte im deponierten Material bzw. deren Relaxation sein. So werden z.B. Korngrenzen, Fremdatom-Einbau oder Oberflächen- bzw. Korngrenzenoxidation als mögliche Spannungsquellen diskutiert [Windischmann, 1987], [Windischmann, 1992]. Bei Sputterprozessen müssen besonders hochenergetische Partikel und die von ihnen ausgelösten Sekundärprozesse wie Stoßkaskaden berücksichtigt werden. Diese Effekte sind in der Regeln nur in dickeren Schichten relevant. In Vielfachschichten mit kleiner Periodenlänge dominieren Grenzflächenspannungen und die durch das epitaktische Wachstum auftretenden Fehlpassungsspannungen.

Die auftretenden Spannungen können eine Größenordnung von GPa erreichen und bei schlechter Haftung zur Ablösung der Filme vom Substrat führen. Im Röntgendiffraktogramm führen sie zu einer Verschiebung der Reflexlagen, die bei elastisch anisotropen Materialien von der Orientierung abhängt.

In der benutzten Triodenmagnetron-Sputteranlage und auch bei Ionenstrahl-Sputteranlagen wachsen bei Raumtemperatur deponierte metallische Filme vorwiegend dichtest gepackt auf. Das bedeutet, dass kubisch-flächenzentrierte Metalle einen sehr großen Anteil (111)-Wachstum, kubisch-raumzentrierte Metalle einen hohen Anteil (110)-Wachstum aufweisen. Die Nishiyama-Wassermann-Beziehung gibt eine Möglichkeit an, wie diese beiden Orientierungen bei entsprechenden Gitterparametern ohne den Einbau von Versetzungen aufeinander aufwachsen können. Abbildung 2.2 zeigt einen entsprechenden Ausschnitt aus zwei Gittern, die entspre- 
chend der Nishiyama-Wassermann-Beziehung aufeinander aufgewachsen sind. Dabei ist die $<100>$-Richtung des raumzentrierten Gitters parallel zur $<110\rangle$ Richtung des flächenzentrierten Gitters und die <011>-Richtung des raumzentrierten Gitters parallel zur $<11 \overline{2}>$-Richtung des flächenzentrierten Gitters. $\mathrm{Zu}$ berücksichtigen ist dabei, dass es aufgrund der kubischen Gitterzellen nicht möglich ist, das kubisch-

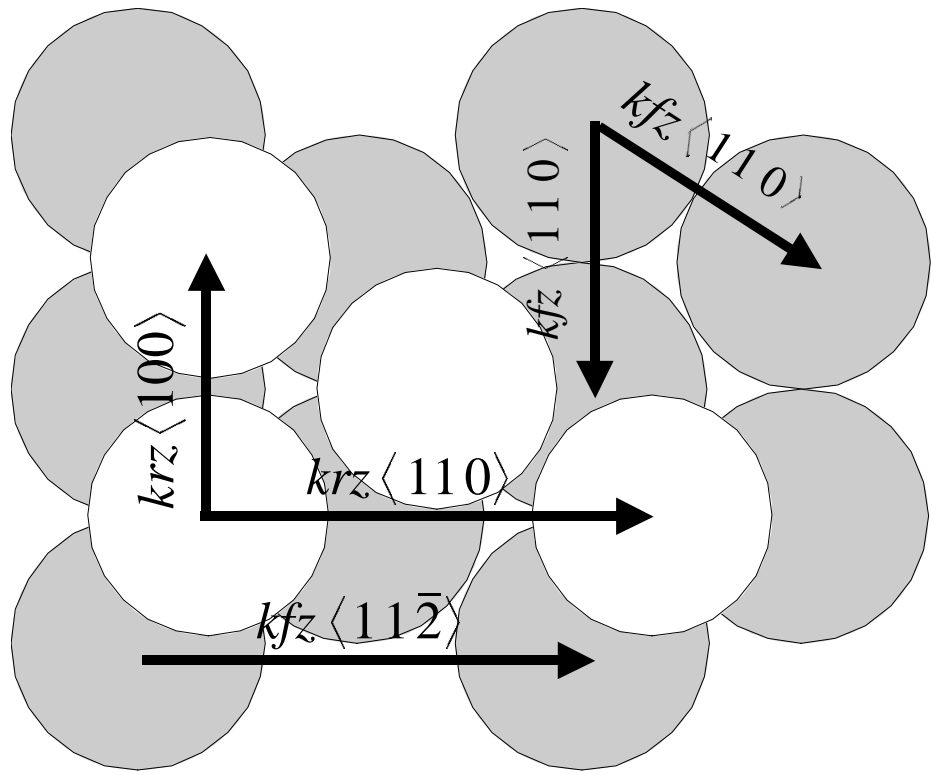
Abbildung 2.2: Modell von Nishiyama-Wassermann: krz(110)-Wachstum auf $k f z(111)$.

raumzentrierte Gitter in $\langle 100\rangle$ - und $\langle 110\rangle$-Richtung zugleich spannungsfrei aufwachsen zu lassen [Haasen K13, 1994].

\subsection{Röntgendiffraktometrie}

Die Röntgendiffraktometrie ist die wahrscheinlich wichtigste und am weitesten verbreitete Methode zur Charakterisierung dünner Schichten. Sie ermöglicht die Identifizierung von Phasen und Texturen und erlaubt auch eine Abschätzung der mittleren Korngröße der Körner, die zu einem Reflex im Röntgendiffraktogramm führen.

\subsubsection{Messungen im 2-Kreis-Diffraktometer}

Die in dieser Arbeit dargestellten Diffraktogramme wurden in Bragg-BrentanoGeometrie in einem Röntgendiffraktometer der Fa. Siemens gemessen, welches ausführlich in [Krebs, 1984] beschrieben wird. Die monochromatische Röntgenstrahlung wird von einer Kobalt- $\mathrm{K}_{\alpha}$-Röhre erzeugt und hat eine Wellenlänge von $1,7903 \AA$. Die Form des Röntgenstrahls ist bei einem Winkel von etwa $\theta=50^{\circ}$ ein Strich mit einer Breite von $1 \mathrm{~mm}$ und einer Länge von $7 \mathrm{~mm}$. Die maximale Auflösung des Geräts bei der Bestimmung der Gitterkonstanten beträgt $\pm 0,01 \AA$. Zusätz- 
lich ist es möglich, den Probenraum mit Hilfe einer Turbomolekularpumpe auf Drücke bis $10^{-6}$ mbar zu evakuieren. Der Probentisch lässt sich auf Temperaturen bis zu $700^{\circ} \mathrm{C}$ heizen. Mit dieser Kombination ist es möglich, kontinuierlich Messungen während der Auslagerungen durchzuführen.

Zur Bestimmung der Desintegrationstemperatur wurden Heizprogramme mit konstanter Erwärmung durchgeführt. Um auf diese Weise sinnvolle Messungen zu erhalten, darf die Temperatur während einer Messung nicht zu stark ansteigen. Zur groben Messung der Desintegrationstemperatur wurde die Temperatur um $0,2^{\circ} \mathrm{C} / \mathrm{min}$ gesteigert. Zur genaueren Festlegung wurden im entsprechenden Temperaturbereich Messungen mit $0,05^{\circ} \mathrm{C} / \mathrm{min}$ durchgeführt. Die Dauer einer Messung lag in allen Fällen bei 10 Minuten.

\subsubsection{Messungen im 4-Kreis-Diffraktometer}

Die Texturuntersuchungen an den Vielfachschichten wurden an einem 4-KreisGoniometer der Fa. Phillips durchgeführt. Auch dieses Gerät arbeitet mit einer Kobalt- $\mathrm{K}_{\alpha}$-Röhre. Im Unterschied zum zuvor vorgestellten 2-Kreis-Diffraktometer ist die Probe hier nicht nur in eine Richtung kippbar, sondern auf einer sogenannten Euler-Wiege in alle Raumrichtungen kippbar.

Zur Messung einer Textur, also einer Verteilung von Kristalliten mit einer bestimmten Orientierung, wird die Bragg-Bedingung für den entsprechenden Kristallreflex fest eingestellt. Dann wird die Probe um einen Winkel $x$ zwischen 0 und $90^{\circ}$ gegen die Einfallsrichtung des Röntgenstrahls gekippt und bei jedem Winkel um $360^{\circ}$ um ihre Normale gedreht ( $\phi$-Scan). Auf diese Weise erhält man eine Verteilung entsprechend orientierter Kristallite für eine Hemisphäre.

\subsubsection{Auswertung der Diffraktogramme}

\section{Gitterkonstanten und Korngrößen}

Die Röntgendiffraktometrie basiert auf der Bragg-Bedingung einer Formel, die beschreibt, unter welchem Winkel ein Röntgenstrahl quasi optisch an einem Kristallgitter reflektiert wird: 


$$
n \cdot \lambda=2 \cdot d_{h k l} \cdot \sin \theta
$$

Dabei gibt $n$ die Ordnung des Maximums, $\lambda$ die Wellenlänge der verwendeten Röntgenstrahlung, $d_{h k l}$ den Netzebenenabstand und $\theta$ den Winkel der Reflexion an. Ist diese Bedingung erfüllt, interferieren die Strahlen, die an den einzelnen Netzebenen reflektiert werden, konstruktiv.

Im Idealfall eines unendlich ausgedehnten Einkristalls und absolut monochromatischer Röntgenstrahlung sollten im Diffraktogramm an den Winkeln, die die BraggBedingung erfüllen, scharfe Reflexe zu sehen sein. Aufgrund der nicht ideal monochromatischen Strahlung und der apparativen Begrenzungen des Diffraktometers ist dies jedoch nicht der Fall. Zudem beeinflusst auch die Mikrostruktur der Probe die Breite der Reflexe. Ist die untersuchte Probe kein Einkristall oder sehr grob kristallin, so kann die Bragg-Bedingung immer weniger genau erfüllt werden, da die destruktive Interferenz für nur leicht aus dem Bragg-Winkel gekippter Strahlen sehr viele Netzebenen benötigt. So führt die endliche Korngröße in polykristallinen Proben zu einer Reflexverbreiterung [Cullity, 1967]. Bei der hier verwendeten Apparatur wird dies bei Kornabmessungen kleiner als $50 \mathrm{~nm}$ [Oelgeschläger, 1984] relevant. Die Halbwertsbreite der Reflexe kann daher dazu zu benutzt werden, die mittlere Größe der Kristallite, die den Reflex erzeugen abzuschätzen. Die ScherrerFormel beschreibt diese Abhängigkeit für runde Körner mittlerer Größe:

$$
D=\frac{0,9 \cdot \lambda}{B \cdot \cos \theta}
$$

Dabei ist die $\mathrm{D}$ die Korngröße, $\lambda$ die Wellenlänge der verwendeten Röntgenstrahlung, B die Halbwertsbreite des Reflexes und $\theta$ der Winkel, an dem der Reflex gemessen wird.

\section{Periodenlänge und Grenzflächenrauigkeit von Vielfachschichten}

Bisher war im Rahmen der Diskussion von Diffraktogrammen nur von solchen Reflexen die Rede, die durch die Reflexion der Röntgenstrahlung am Kristallgitter verursacht werden. Aufgrund eines typischen Netzebenenabstandes im Kristallgitter von etwa 1-3 A liegen die Reflexe im Winkelbereich zwischen $30^{\circ}$ und $120^{\circ}$. Im Falle von Vielfachschichten erzeugt man jedoch ein weiteres Gitter mit einem 
Netzebenenabstand, der der Periodenlänge des Schichtpaketes entspricht. Aufgrund der deutlich größeren Gitterkonstante im Bereich einiger Nanometer sind die BraggReflexe dieses Lagengitters im Bereich einiger Grad zu finden und werden als Kleinwinkelreflexe bezeichnet.

Das Auftreten solcher Kleinwinkelreflexe ist ein Maß für die Güte der Grenzflächen der obersten Doppellagen, da der Röntgenstrahl bei einem so kleinen Einfallswinkel und einer Eindringtiefe von $5 \mu \mathrm{m}$ nur knapp $100 \mathrm{~nm}$ tief in die Probe eindringt. Desweiteren muss die Welligkeit sehr klein sein, da die Periodizität in Richtung des Röntgenstrahls vorhanden sein muss. Bei Vielfachschichten mit einer Periodenlänge von $10 \mathrm{~nm}$ durchdringt der Strahl nur alle $500 \mathrm{~nm}$ eine Doppellage. Ist das Schichtpaket auf dieser Skala nicht glatt, sind keine Überstrukturen im Kleinwinkelbereich zu sehen. Der kleine Einfallswinkel ist auch dafür verantwortlich, dass bei Periodenlängen von $10 \mathrm{~nm}$ und mehr manchmal der Reflex erster Ordnung schwächer als der zweiter Ordnung ist, da durch die Vergrößerung des Winkels für den Reflex zweiter Ordnung mehr Doppellagen an der konstruktiven Interferenz beteiligt sind.

\section{Das Stufenmodell und seine Erweiterung auf Dickenfluktuationen}

In Diffraktogrammen von Vielfachschichten finden sich neben den Kleinwinkelreflexen noch weitere Besonderheiten im Bereich der Kristallreflexe. Hier wird beobachtet, dass die Kristallreflexe moduliert sind und in ihrer Nähe weitere Reflexe auftreten, die als Satellitenreflexe bezeichnet werden. Diese Überstruktur entsteht mathematisch durch eine Faltung der Kristallgitterreflexe und der Vielfachschichtreflexe hoher Ordnung. Daher kann aus dem Abstand der Überstrukturreflexe direkt auf die Periodenlänge geschlossen werden, wenn man die Bragg-Bedingung für Reflexe entsprechend hoher Ordnung für das Vielfachschichtgitter zugrunde legt. Aus den Intensitäten und den Lagen der Überstrukturreflexe kann man jedoch noch sehr viel weitergehende Aussagen über die Rauigkeiten, die Gitterparameter und die Kohärenz des Schichtpaketes ableiten [Hamp, 1997].

Dazu kann das sogenannte Stufenmodell angewendet werden, bei dem zunächst einzelne Gitterebenen der entsprechenden Metalle entsprechend der Einzellagendicke übereinander gestapelt werden. Im Modell hat man dann N Doppellagen, die 
jeweils $\mathrm{N}_{\mathrm{a}}$ Netzebenen der Atomsorte A mit Netzebenenabstand $\mathrm{d}_{\mathrm{a}}$ und $\mathrm{N}_{\mathrm{b}}$ Netzebenen der Atomsorte B mit Netzebenenabstand $d_{b}$ enthalten. Der Netzebenenabstand zwischen den Metallen wird mit $\left(\mathrm{d}_{\mathrm{a}}+\mathrm{d}_{\mathrm{b}}\right) / 2$ abgeschätzt. Durch phasengerechte Überlagerung der an diesem künstlichen Gitter gestreuten Wellen lässt sich der eindimensionale Strukturfaktor F in Abhängigkeit des Streuvektors $s=(4 \pi \sin \theta) / \lambda$ berechnen, der die Winkel- und Wellenlängeninformation enthält. Die Intensität ergibt sich dann als Betrag der komplexen Größe F: $I=F^{*}(s) F(s)$.

Dieses Modell gibt sehr gut die Lage der Reflexe und die Gitterparameter der beteiligten Metalle wieder. Um Informationen über die Rauigkeit der Vielfachschicht zu erhalten, muss das Modell jedoch noch er-

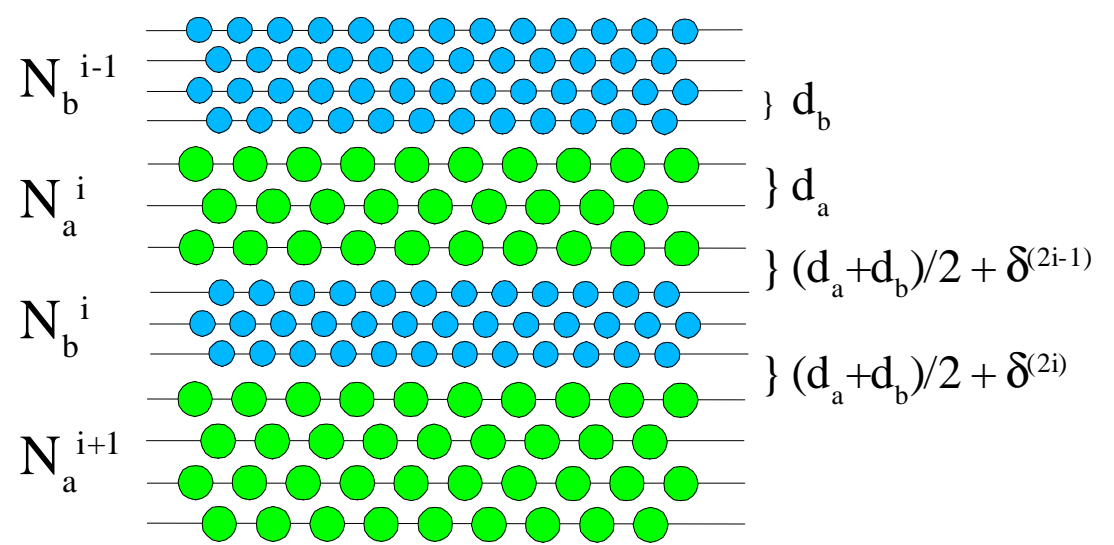

Abbildung 2.3: Stufenmodell für Überstrukturen mit der Erweiterung für diskrete und kontinuierliche Fluktuationen. weitert werden

(Abbildung 2.3), indem zugelassen wird, dass die Zahl der Netzebenen pro Lage $\mathrm{N}_{\mathrm{a}}$ und $\mathrm{N}_{\mathrm{b}}$ von Lage zu Lage variieren und dass der Netzebenenabstand an den Korngrenzen von Lage zu Lage um einen Wert $\delta^{i}$ unterschiedlich sein darf. Die fluktuierenden Werte sind dabei normalverteilt um die Werte $\overline{N_{a}}$ und $\overline{N_{b}}$ bzw. $\delta$. Setzt man diese Halbwertsbreiten der Verteilungen gleich Null, geht das Modell wieder in das einfache Stufenmodell über. Dieses Modell wurde zuerst von [Loquet, 1989] vorgeschlagen. Mit Hilfe dieses Modells lassen sich die meisten Überstrukturen gut anpassen. In Abbildung 2.4 ist zur Demonstration die Anpassung an eine Kupfer/Kobalt Vielfachschicht gezeigt. Die Anpassung ergab in diesem Fall eine Periodenlänge von $5 \mathrm{~nm}$, wobei die Lagendicke von Kupfer bei $4 \mathrm{~nm}$ und die von Kobalt bei $1 \mathrm{~nm}$ lag. Der Gitterparameter von Kupfer liegt nahezu auf dem Wert des Massivmaterials. Der Gitterparameter des Kobalt ist leicht vergrößert, was durch ein verspanntes Aufwachsen auf den Kupferlagen zu erklären ist. Die Rauigkeit dieses kohärenten Schichtpaketes liegt in diesem Fall bei etwa $1 \mathrm{~nm}$. 


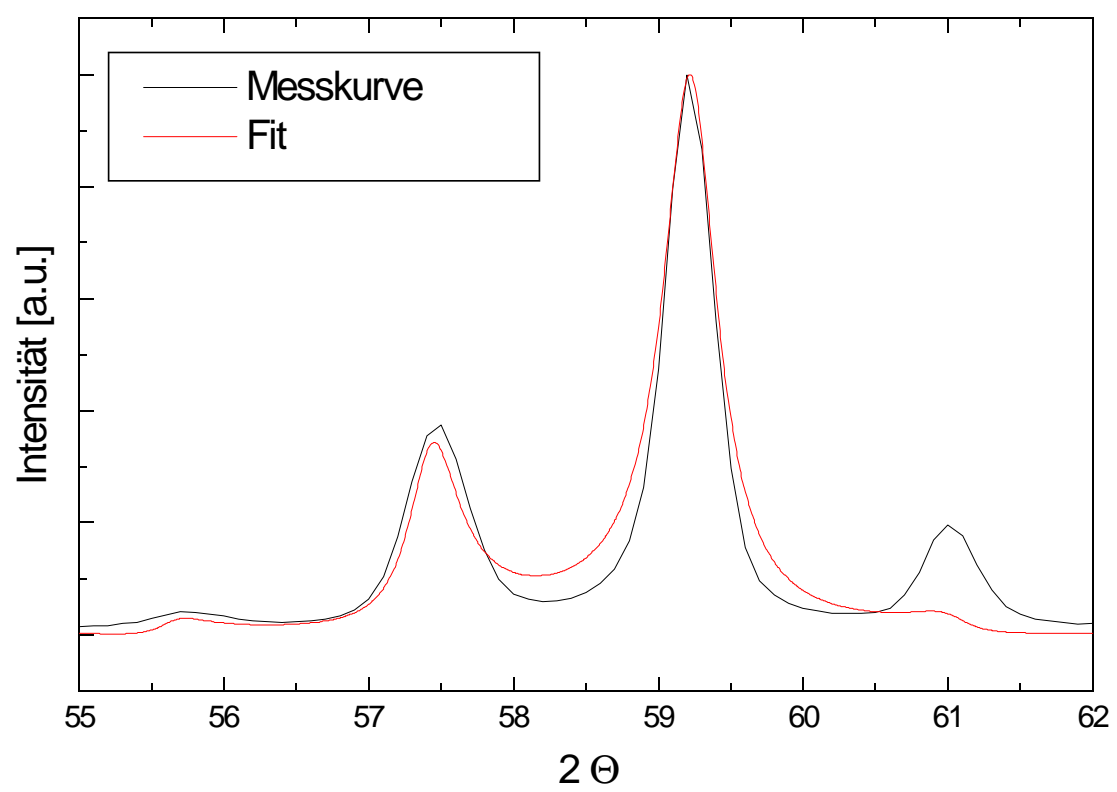

Abbildung 2.4: Anpassung an eine Überstruktur von Cu/Co-Vielfachschichten. Hier die Überstruktur der Cu und Co (220)-Reflexe.

\subsubsection{Transmissionselektronenmikroskopie}

Zur hochauflösenden Charakterisierung von dünnen Schichten ist die Transmissionselektronenmikroskopie ein sehr wichtiges Instrument. Die in dieser Arbeit gezeigten Bilder wurden an einem von der Fa. Phillips hergestellten Transmissionselektronenmikroskop (TEM) des Typs Philips EM 420 ST gemacht.

\section{Z-Kontrast}

Da die Methode der Transmissionselektronenmikroskopie allgemein bekannt ist, soll hier vor allem auf eine Erweiterung an diesem Gerät eingegangen werden, die Hollow-Cone-Dunkelfeldbeleuchtung (Z-Kontrast). Mit Hilfe dieser Methode ist es möglich, ein massensensitives Bild der Probe mit einer Auflösung von etwa einem Nanometer zu erzeugen. Um diese ordnungszahlabhängige (Ordnungszahl Z) Abbildung zu erhalten, benutzt man zur Abbildung nur die unter großem Winkel gestreute Elektronen. Die unter Streuwinkeln von $\Theta \sim 150 \mathrm{mrad}$ gestreuten Elektronen wurden überwiegend inkohärent an den Atomkernen gestreut, wodurch 
Beugungseffekte weitgehend vermieden werden. Die inkohärente Streuung an den Atomkernen hängt von der Ordnungszahl und von der Anzahl der Streuzentren, also der Dicke der Probe, ab. Aufgrund der geringen Intensität der unter den großen Winkeln gestreuten Elektronen ist eine lange Belichtungszeit der so aufgenommen Bilder erforderlich. Durch Benutzung der Objektivblende tragen nur die um den Winkel $\Theta$ gestreuten Elektronen zur Abbildung bei. Abbildung 2.5 zeigt eine solche Aufnahme von einer 30 Minuten bei $320^{\circ} \mathrm{C}$ ausgelagerten

Eisen/Gold-(2 nm/10 nm)-Vielfachschicht.

Die dabei gebildeten Eisenpartikel und auch das Loch in der Probe (rechts oben) sind aufgrund ihrer schwachen Streuung hell abgebildet. Dagegen ist die Goldmatrix als dunkle

Abbildung 2.5: Z-Kontrast-Bild einer ausgelagerten Eisen/Gold-Vielfachschicht. Hell ist das schwach streuende Eisen, dunkel das Gold zu sehen. Umgebung zu erkennen. Die Diskussion und genaue Darstellung dieser Ergebnisse wird in Kapitel 4 vorgenommen.

In [Ewert, 1998] und [Schleiwies, 1998] finden sich weitere Details zu diesem Verfahren.

\section{Querschnittspräparation}

Mikrostrukturuntersuchungen an Vielfachschichten sind nur dann besonders aussagekräftig, wenn die Lagenstruktur selber abgebildet wird. Dazu ist es nötig, die TEM-Proben einer Querschnittspräparation zu unterziehen. Dabei werden von der Probe zwei etwa 1,9 mm breite Streifen abgeschnitten und mit der beschichteten Seite aufeinander geklebt. Dann wird diese Probe um zwei Kupferhalbzylinder ergänzt und in ein Edelstahlröhrchen eingefasst (Abbildung 2.6). Zum Einkleben wird dabei ein spezieller Kleber der Fa. Gatan benutzt.

Von dem Röhrchen, das die Probe enthält, werden mit der Innenlochsäge $500 \mu \mathrm{m}$ 


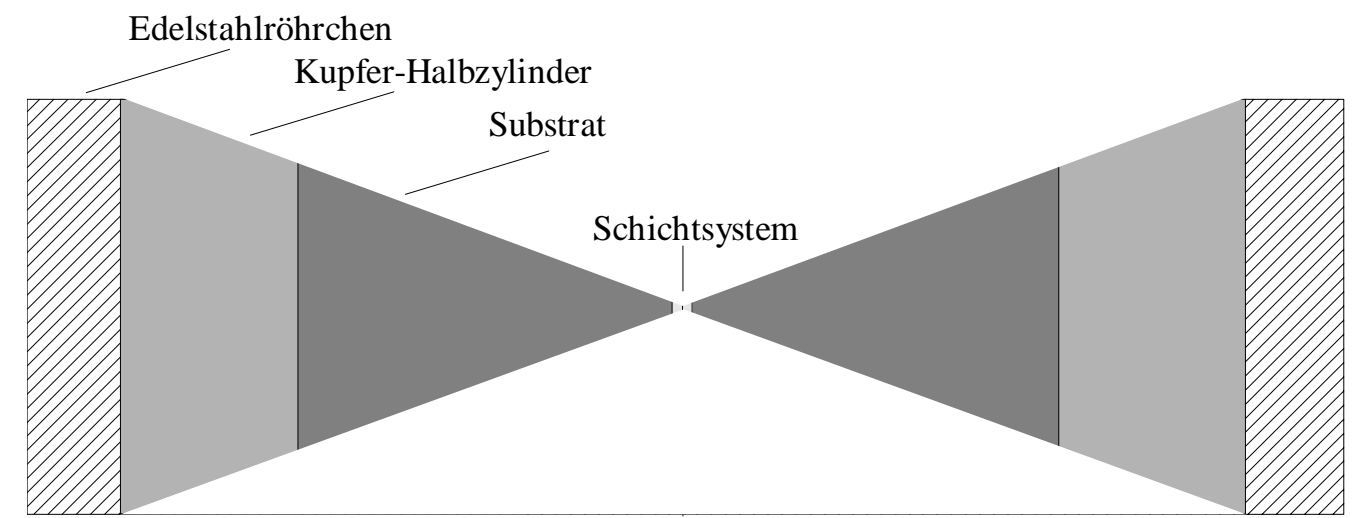

Abbildung 2.6: Skizze des Querschnitts einer für das TEM präparierten Probe mit Umgebung.

dicke Streifen abgeschnitten und dann mit Schleifpapier auf $100 \mu \mathrm{m}$ weiter gedünnt. Ein konisch geschliffenes Stahlrad dünnt die Probe weiter zur Mitte keilförmig, so dass sie in der Mitte schließlich eine Dicke von $20 \mu \mathrm{m}$ hat. An die mechanische Dünnung schließt sich die Dünnung mit einem Ar-Ionenstrahl an, mit dem ein Loch in die Probe geätzt wird. Der Einschusswinkel der Ar-Ionen liegt dabei zwischen $13^{\circ}$ und $30^{\circ}$ von der Präparatebene her gemessen. Die Beschleunigungsspannung beträgt $3 \mathrm{kV}$ bis $6 \mathrm{kV}$. Spezielle Bewegungen der Probe verhindern dabei, dass der Keil abstumpft [Hartung, 1996], [Ewert et al., 1997].

\subsection{Röntgenmikroskopie}

Die Röntgenmikroskopie ist eine relativ neue Methode zur mikrostrukturellen Untersuchung von Festkörpern. Sie fällt unter die Kategorie der Durchstrahlungsmethoden. Das Gerät wird mit Synchrotonstrahlung des Bessy in Berlin betrieben ${ }^{1}$ [Schmahl et al., 1996].

Da Röntgenstrahlung in Metallen deutlich weniger absorbiert wird als Elektronen, darf die durchstrahlte Schicht je nach Metall etwa $100 \mathrm{~nm}$ dick sein und bedarf daher keiner aufwendigen Präparation. Für die vorgestellten Messungen wurden spezielle Vielfachschichten mit nur wenigen Doppellagen (zwischen 3 und 10) und einer Passivierungsschicht auf jeder Seite des Schichtpaketes gesputtert. Nach Entfernen des Substrats konnten diese Proben direkt durchstrahlt werden. Ein Ersatz für die Transmissionselektronenmikroskopie ist die Methode jedoch nicht, da zum

1 Die in dieser Arbeit gezeigten röntgenmikroskopischen Bilder wurden freundlicher Weise von Herrn Dr. Rudolph vom Institut für Röntgenphysik der Universität Göttingen gemacht. 

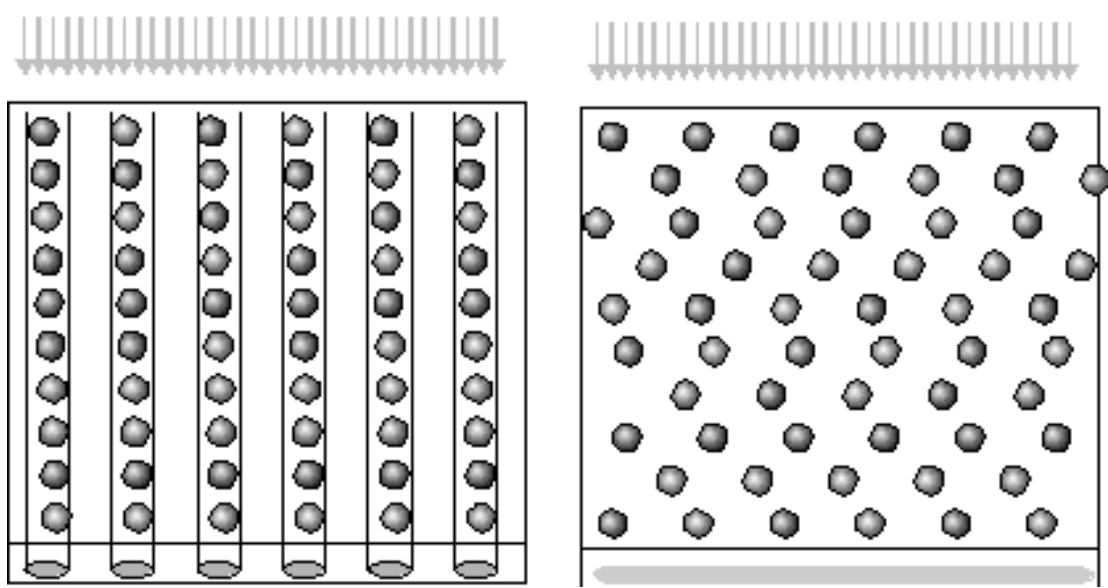

Abbildung 2.7: Kontrast bei Durchstrahlung von statistisch angeordneten Partikeln und in Säulen angeordneten Partikeln.

einen ein Pixel der CCD-Aufnahmekamera bei maximaler Vergrößerung nur knapp $10 \mathrm{~nm}$ entspricht und zum anderen nur parallel zur Schichtnormalen gemessen werden kann. Die Auflösung hängt dabei sehr empfindlich von der Qualität der eingesetzten Zonenplatten ab. Trotz dieser begrenzten Auflösung ermöglicht sie, eine Statistik über eine große Fläche der Probe zu erstellen und den Grad der Ordnung der gebildeten Partikel senkrecht zur Schicht zu messen.

Eine solche direkte Durchstrahlungsmethode bildet bei vollständig intakten Vielfachschichten eine gleichmäßige Färbung ab. Das heißt, dass die Probe die Röntgenstrahlung an allen Stellen gleichermaßen stark absorbiert. Findet nun eine Desintegration der Vielfachschicht statt, ist nicht selbstverständlich ein Kontrast zu

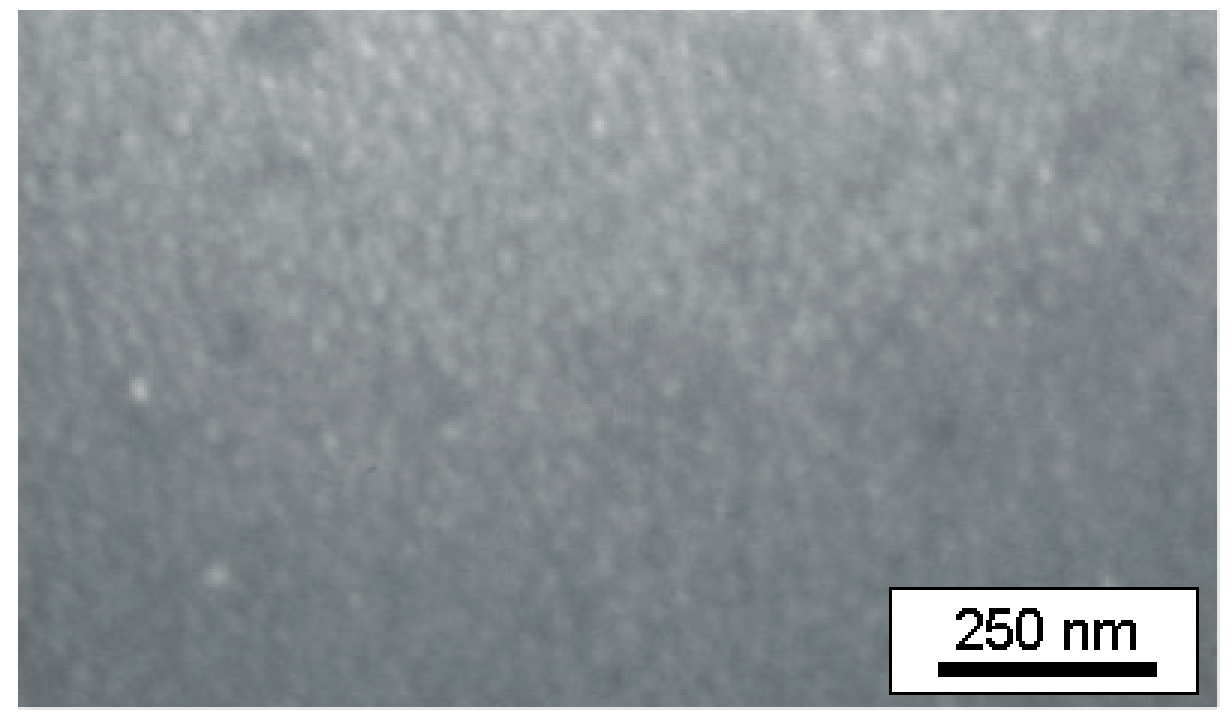

Abbildung 2.8: Röntgenmikroskopische Abbildung von Ketten aus Eisenpartikeln (senkrecht zur Schicht) in einer Goldmatrix. 
erkennen. Dazu stelle man sich eine Vielfachschicht bestehend aus 10 Doppellagen vor, in der sich die Partikel in jeder Lage statistisch gebildet haben (Abbildung 2.7 rechtes Bild). In diesem Fall ist die mittlere Absorption der Schicht ähnlich wie im ursprünglichen Zustand fast konstant, da im Mittel an jeder Stelle der Probe wieder gleich viel Metall von beiden Sorten durchstrahlt wird. Das ergibt sich ganz zwangsläufig aus der Konstanz der Volumina beider Metalle. Das bedeutet aber auf der anderen Seite, dass im Falle der Abbildung eines Kontrastes wie ihn Abbildung 2.8 zeigt, keine statistische Anordnung der Partikel in der Probe vorliegen kann. Solche ovale, helle Kontraste können nur dadurch entstehen, dass sich die Partikel tendenziell übereinander anordnen und so mehrere Partikel einen gemeinsamen Kontrast erzeugen. Die ovale Form der Kontraste deutet darauf hin, dass die Partikel nicht exakt übereinander, sondern leicht gegeneinander versetzt in der Probe liegen. Aufgrund der Intensität der einzelnen Spots kann auf die Zahl der Nanopartikel geschlossen werden. Ist ein Spot besonders hell, liegen viele Partikel übereinander.

\subsection{Schichtdickenmessung}

Die Dicke der hergestellten Vielfachschichten wurden mechanisch mit einem Mikroabtastsystem der Fa. Feinprüf-Perthen gemessen. Dabei wird eine Nadel mit einer sehr geringen Auflagekraft von $0,8 \mathrm{mN}$ über die Probe geführt und die vertikale Auslenkung der Nadel gemessen, indem ein Induktionssignal aufgenommen wird. Auf diese Weise lassen sich real Höhenunterschiede von bis zu $30 \mathrm{~nm}$ messen. Die laterale Auflösung wird durch den Radius der Nadelspitze von $5 \mu \mathrm{m}$ begrenzt.

Um diese Messungen durchführen zu können, wird während der Deposition ein Teil des Substrats abgedeckt. Zur Schichtdickenmessung fährt man die Nadel über die Abschattungskante und erhält aus dem Signal direkt die Dicke der Vielfachschicht. 


\section{Mikrostrukturuntersuchungen an Vielfachschichten}

Zur Untersuchung von Mechanismen der Desintegration von Vielfachschichten ist eine gute Kenntnis der Mikrostruktur im wie-hergestellten Zustand unerlässlich. Diese wird in diesem Kapitel für die Systeme Niob/Kupfer, Eisen/Gold, Eisen/Silber, Kobalt/Kupfer und Kobalt/Kohlenstoff dargestellt. Unter Desintegration soll zunächst jede Zerstörung der periodischen Struktur verstanden werden. Im Rahmen einer Diplomarbeit [Troche, 1996] zur Gestaltinstabilität von Niob/Kupfer-Vielfachschichten zeigte sich bereits, dass die Grenzflächen einen starken Einfluss auf die Veränderung der Mikrostruktur während der Auslagerung haben. Durch die Grenzflächen wird je nach System mehr oder weniger viel Energie in das Schichtpaket eingebracht. Ist diese Energie konstant entlang einer perfekt glatten Grenzfläche, kann ein solcher Zustand zunächst durchaus stabil sein. Durch lokale Variationen der Grenzflächenenergie kann jedoch ein Materietransport parallel zur Grenzfläche angeregt werden. Solche Variationen der Grenzflächenenergie können verschiedene Ursachen haben. Neben lokalen Variationen der Grenzflächenkrümmung können z.B. auch Variationen in der mechanischen Grenzflächenspannung zu einer lokalen Veränderung der Grenzflächenenergie führen.

Um die verschiedenen möglichen Einflüsse auf die Desintegration separieren zu können, wurden zur Untersuchung Systeme nicht mischender Metalle ausgesucht, die die verschiedenen Aspekte möglichst gut abdecken. Besonderes Augenmerk wurde dabei auf den Betrag der Grenzflächenenergie gerichtet. Im System Niob/Kupfer wurden inkohärente Grenzflächen gefunden, die energetisch im Bereich einiger $\mathrm{J} / \mathrm{m}^{2}$ liegen. Deutlich größere Grenzflächenenergien sind in kohärenten, aber stark verspannten Grenzflächen zu erwarten, wie sie aus der Literatur für die Systeme Eisen/Gold und Eisen/Silber bekannt sind (einige $10 \mathrm{~J} / \mathrm{m}^{2}$ ). Grund ist die Fehlpassung beim Wachstum von raumzentrierten auf flächenzentrierten Metallen (Tabelle 3.1). Aufgrund der ähnlichen Gitterkonstanten von Gold und Silber (Tabelle 3.1, [Kittel, 1993]) und damit ähnlicher

\begin{tabular}{|c|c|c|}
\hline Metall & NN-Abstand $[\AA]$ & Struktur \\
\hline \hline $\mathrm{Nb}$ & 2,86 & $\mathrm{krz}$ \\
\hline $\mathrm{Cu}$ & 2,56 & $\mathrm{kfz}$ \\
\hline $\mathrm{Fe}$ & 2,48 & $\mathrm{krz}$ \\
\hline $\mathrm{Ag}$ & 2,89 & $\mathrm{kfz}$ \\
\hline $\mathrm{Au}$ & 2,88 & $\mathrm{kfz}$ \\
\hline $\mathrm{Co}$ & 2,51 & $\mathrm{hdp}$ \\
\hline
\end{tabular}

Tabelle 3.1: Nächste Nachbarabstände und Gitterstrukturen der verwendeten Metalle [Kittel, 1993]. 
struktureller Grenzflächenenergie ermöglichen vergleiche der Systeme Eisen/Gold und Eisen/Silber zudem Einflüsse anderer Faktoren, wie z.B. der Krümmung der Grenzfläche, zu erkennen, welche in der Lage sind, die Desintegration zu beeinflussen. Ein System, das für kohärente Grenzflächen mit kleinen Energien bekannt ist, ist das System Kobalt/Kupfer (einige $100 \mathrm{~mJ} / \mathrm{m}^{2}$ ). Es eignet sich gut als Vergleichsystem, da es zum einen den Bereich kleiner Grenzflächenenergien abdeckt und zum anderen eines der am besten untersuchten Systeme in der Literatur ist. Um den Einfluss der kristallinen Struktur der Schichten und ihrer Korngrenzen auf die Stabilität der Schichten abschätzen zu können, wurde zudem das System Kobalt/Kohlenstoff untersucht, in dem der Kohlenstoff nach der Deposition in amorpher Form vorliegt. Grenzflächenenergien solcher Systeme mit amorphen Lagen sind ebenfalls eher gering, da es an ihren Grenzflächen keine Fehlpassungsspannungen gibt.

\subsection{Das System Niob/Kupfer}

In früheren Untersuchungen im Rahmen einer Diplomarbeit [Troche, 1996] wurde die Mikrostrukturveränderung von Niob-Kupfer-Vielfachschichten während einer thermischen Behandlung ausführlich charakterisiert. Um die Daten zur allgemeinen Diskussion der Gestaltinstabilität von Vielfachschichten präsent zu haben, werden die wichtigsten Ergebnisse der Diplomarbeit hier und im Kapitel über die Gestaltinstabilität dargestellt. Abbildungen, die nicht als Zitat gekennzeichnet sind, sind nach Fertigstellung der Diplomarbeit entstanden.

Für die ersten Untersuchungen zur Desintegration von Multilagen erwies sich dieses System als überaus geeignet, da das System eine sehr breite Mischungslücke zeigt, die bis zu sehr hohen Temperaturen stabil ist (Abbildung 3.1). So löst sich bei $1080^{\circ} \mathrm{C}$ nur 0,1 Prozent Niob im Kupfer und etwa 1,2 Prozent Kupfer in Niob. Für den in experimentell zugänglichen Temperaturbereich von bis $\mathrm{zu} 650^{\circ} \mathrm{C}$ ist eine thermodynamische Mischung der Metalle demnach auszuschließen. Feldionenmikroskopische Messungen [Schleiwies, 1998] bestätigen dies sowohl für Multilagen als auch für ausgelagerte Proben. 


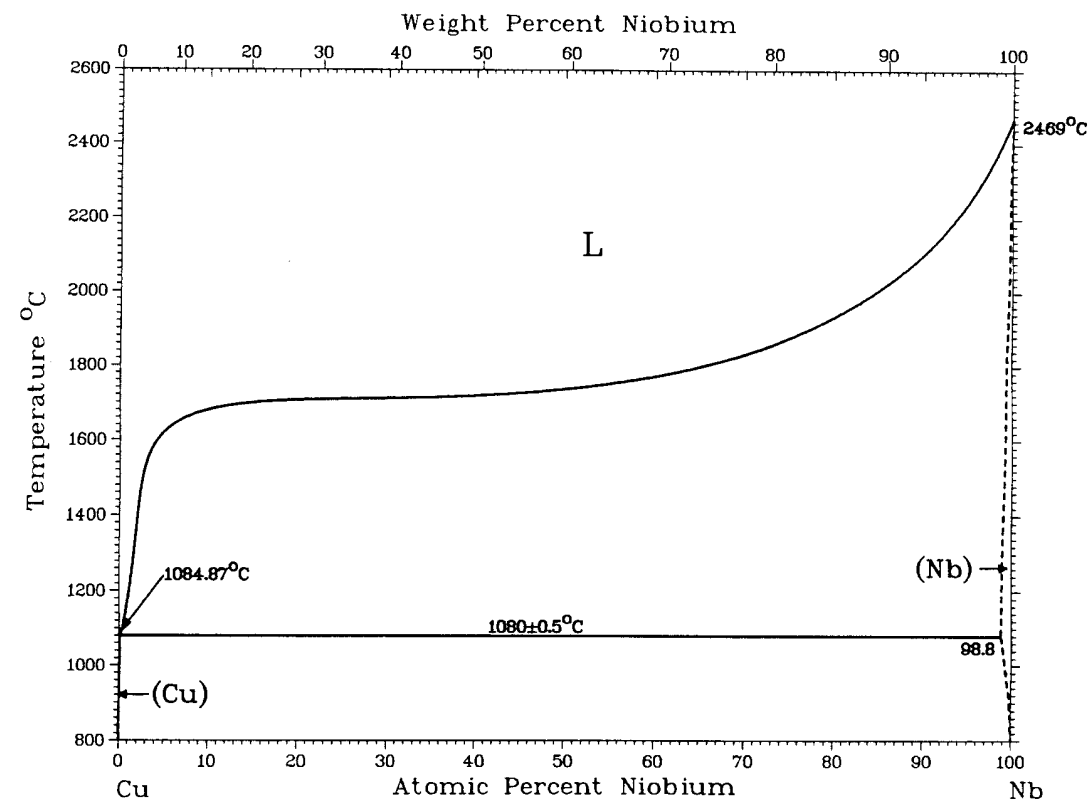

Abbildung 3.1: Phasendiagramm Niob/Kupfer.

Die unbehandelten Niob/Kupfer-Vielfachschichten weisen eine bevorzugte (110)Textur für Niob und eine (111)-Textur für Kupfer, sowie Überstrukturreflexe auf. Nach der Nishiyama-Wassermann-Beziehung müssten die Niobschichten in $\langle 110\rangle$ Richtung eine Fehlpassung von $-20 \%$ und in $\langle 100\rangle$-Richtung von $-10 \%$ aufweisen. Die Analyse der Überstruktur zeigt aber, dass das Niob-Gitter um $7 \%$ gedehnt ist und dass das Schichtpaket nicht kohärent aufwächst. Die Grenzfläche hat hier also eher den Charakter einer Großwinkelkorngrenze. Eine weitergehende Abschätzung der Grenzflächenenergie wird später in Kapitel 6.2.1 durchgeführt.

Die Korngröße innerhalb der Kupferlagen entspricht in etwa der Lagendicke, sie

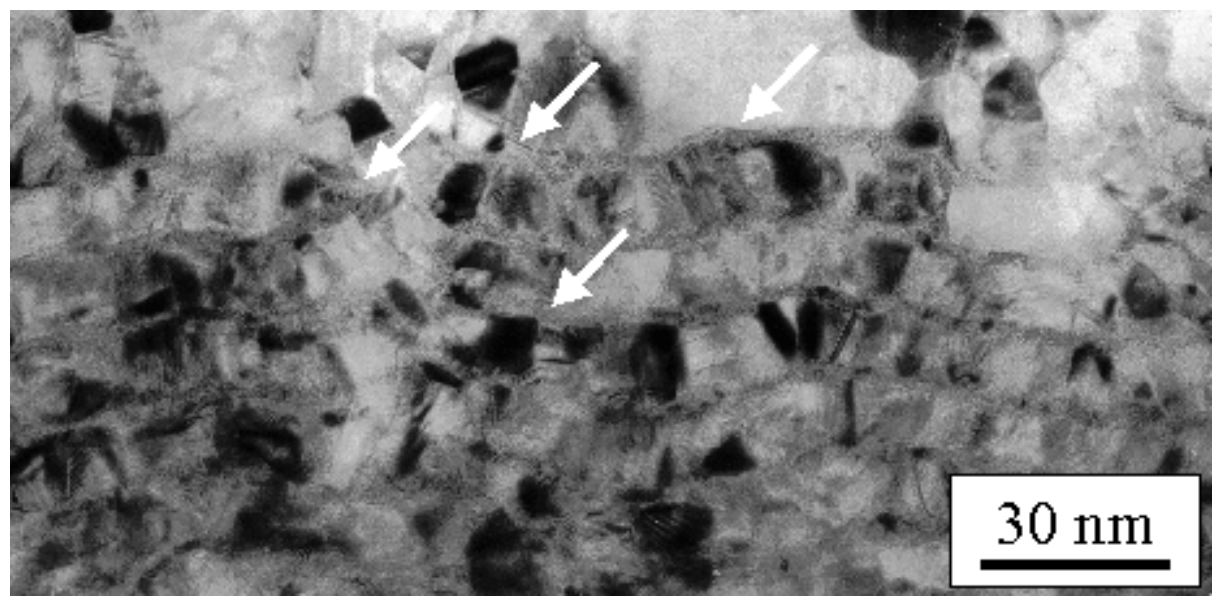

Abbildung 3.2: $\mathrm{Nb} / \mathrm{Cu}$-Vielfachschicht $(2 \mathrm{~nm} / 10 \mathrm{~nm})$. Es ist eine langwellige und eine kurzwellige Rauigkeit zu erkennen. Die kurzwellige Rauigkeit (helle Pfeile) setzt sich nicht über die Lagen hinweg fort. 
liegt im Bereich zwischen 5 und $20 \mathrm{~nm}$. Die Rauigkeit der Grenzflächen baut sich langsam auf und vergrößert sich mit zunehmender Schichtdicke. Neben einer langwelligen Rauigkeit, die sich zum Teil über die Lagen fortsetzt, gibt es eine kurzwellig Rauigkeit, die durch die polykristalline Kornstruktur verursacht wird (Abbildung 3.2, helle Pfeile). Diese kurzwellige Rauigkeit setzt sich nicht fort und baut sich innerhalb einer Periode wieder ab.

Bei Schichten, die in der verwendeten Sputteranlage hergestellt wurden, wird eine Kippung der Textur aus der senkrechten Lage beobachtet. Die Ursache dafür ist der Winkel, unter dem die gesputterten Atome auf die Substrate treffen (siehe Montage der Quellen Abbildung 2.1). Die Stärke der Kippung ist davon abhängig, wie der Winkel zwischen Substrat und Sputterquelle während der Deposition war und ob mehrere Quellen während der Deposition in Betrieb waren. Werden zum Beispiel zwei Metalle mit gleicher Einzellagendicke und gleichen Einfallswinkeln hergestellt, so wachsen die dichtest gepackten Ebenen fast parallel zum Substrat auf. Texturuntersuchungen zeigen für Niob/Kupfer-Schichtpakete neben einer Drahttextur der Filme auch diese Kippung, die in der Regel kleiner als $12^{\circ}$ ist. Außerdem ist in den Polfiguren zu erkennen, dass der Winkel der Niob-(110)-Ebenen deutlich durch den Winkel der dickeren Kupfer-(111)-Lagen dominiert wurde. Es bildeten sich jedoch beim Wachstum des Niob Nebenmaxima, die eine Tendenz des Niob andeuten, in Richtung der Niobquelle $\mathrm{zu}$ wachsen. In Abbildung 3.3 ist eine Niob-(110)Textur zu sehen, deren Hauptreflex um $x=8^{\circ}$ aus der senkrechten gekippt ist.

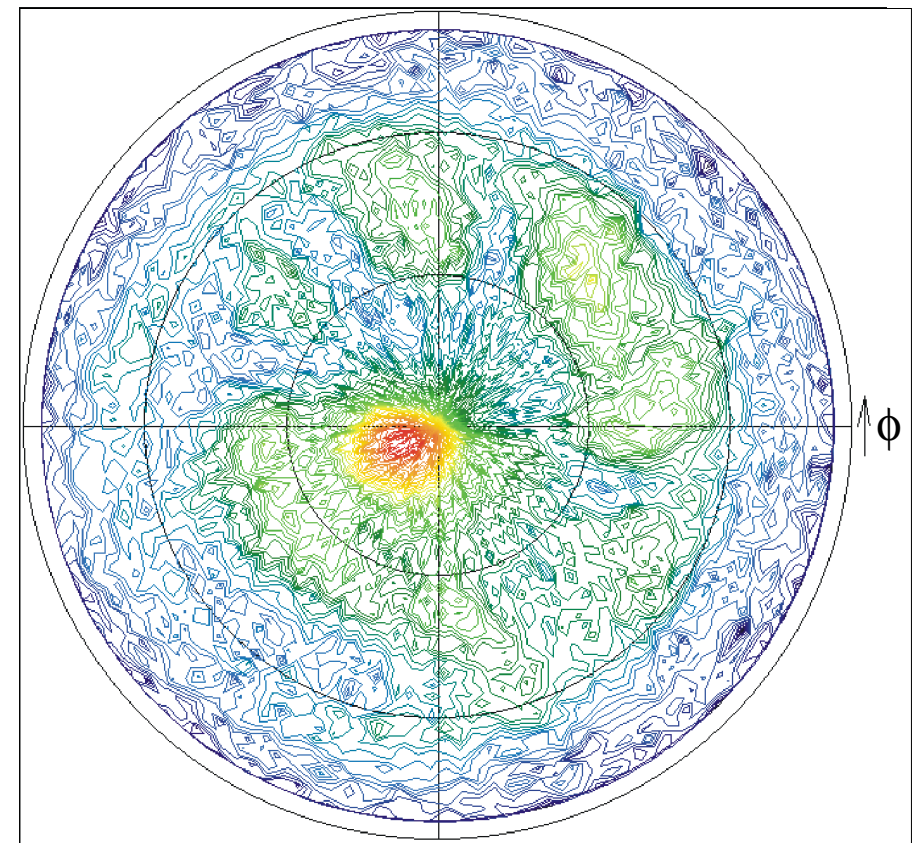

Abbildung 3.3: Polfigur des (110)-Niob-Reflexes in $\mathrm{Nb} / \mathrm{Cu}$-Multilagen $(2 \mathrm{~nm} / 10 \mathrm{~nm})$. 


\subsection{Die Systeme Eisen/Silber und Eisen/Gold}

Um die Ursachen der Desintegration genauer eingrenzen zu können, wurde versucht, zwei nicht mischende Systeme zu finden, die zum einen ähnliche kristalline Strukturen haben, sich aber von ihren chemischen Eigenschaften her deutlich voneinander unterscheiden und zum anderen eine kleinere Gitterfehlpassung zeigen als Niob und Kupfer. Auf diese Weise können gegebenenfalls die Folgen von starken mechanischen Grenzflächenspannungen aufgrund einer kohärenten Grenzfläche beobachten werden.

Die

Eisen/Silber

Systeme

Eisen/Gold erfüllen

diese Eigenschaften. Die Gitterparameter der kubisch-flächenzentrierten Edelmetalle

Gold $(4,08 \AA)$ und Silber $(4,09 \AA)$ weichen nur um etwa 0,2 Prozent voneinander ab. Ein Blick auf die Phasendiagramme (Abbildung 3.4) offenbart jedoch grundlegende Unterschiede in der Chemie der Metalle. Während im System Eisen/Silber vollständige Entmischung bis $\mathrm{zu}$ einer Temperatur von $911{ }^{\circ} \mathrm{C}$ beobachtet wird, liegt im System Eisen/Gold schon bei $300^{\circ} \mathrm{C}$ eine deutliche Randlöslich-
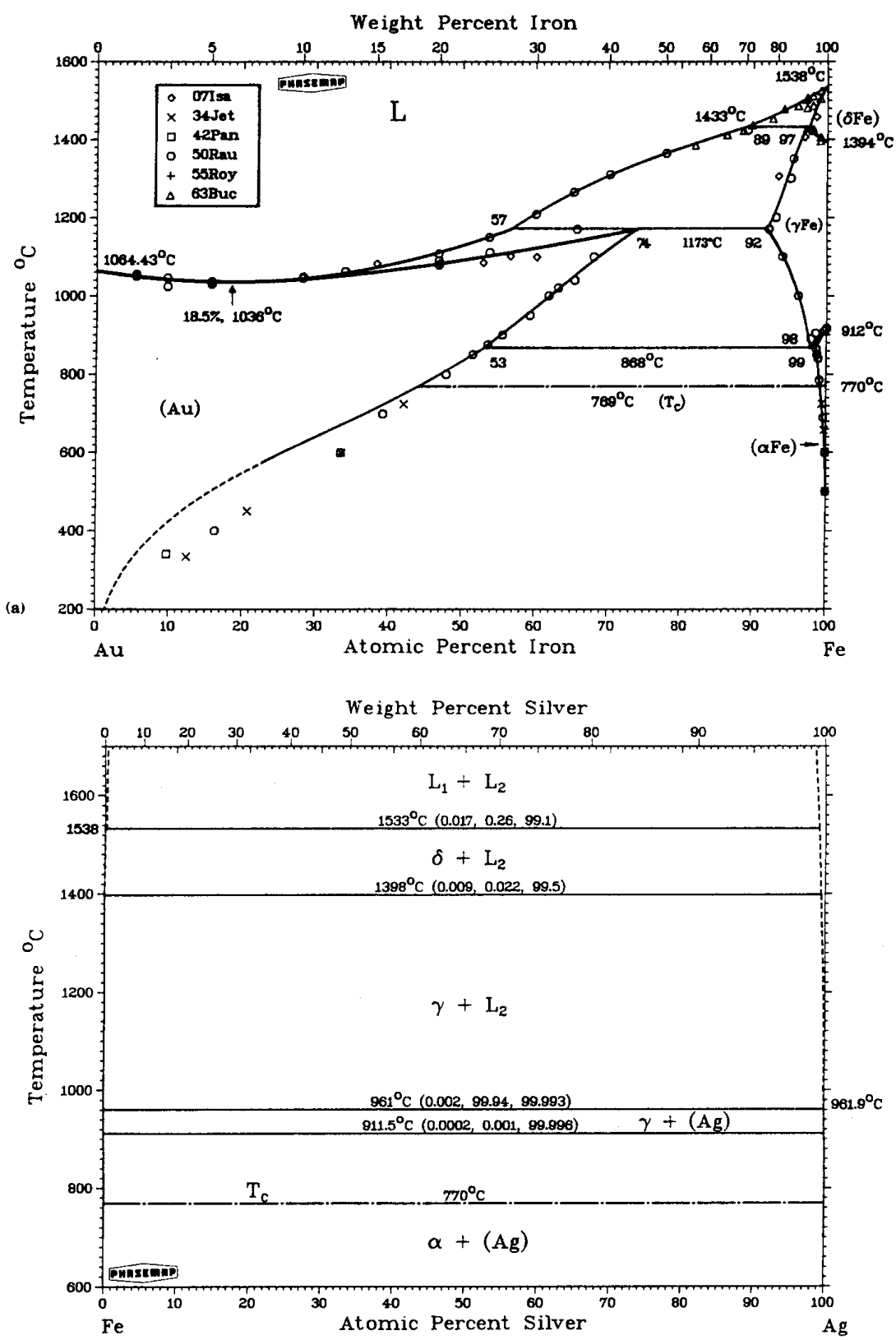

Abbildung 3.4: Phasendiagramme der Systeme Eisen/Gold und Eisen/Silber [Moffatt, 1984]. 
keit vor. Die Mischungslücke verkleinert sich asymmetrisch von der Goldseite her. Bei 400 ${ }^{\circ} \mathrm{C}$ lösen sich etwa 10 At.\% Eisen im Gold. Die Löslichkeit von Gold im Eisen liegt bei dieser Temperatur noch deutlich unter einem Prozent. Die Gitterfehlpassung zwischen Eisen und Gold bzw. Silber ist, für die experimentell beobachtete Nishiyama-Wassermann-Beziehung [Bruce et al., 1978] zwar relativ groß, jedoch deutlich ge-

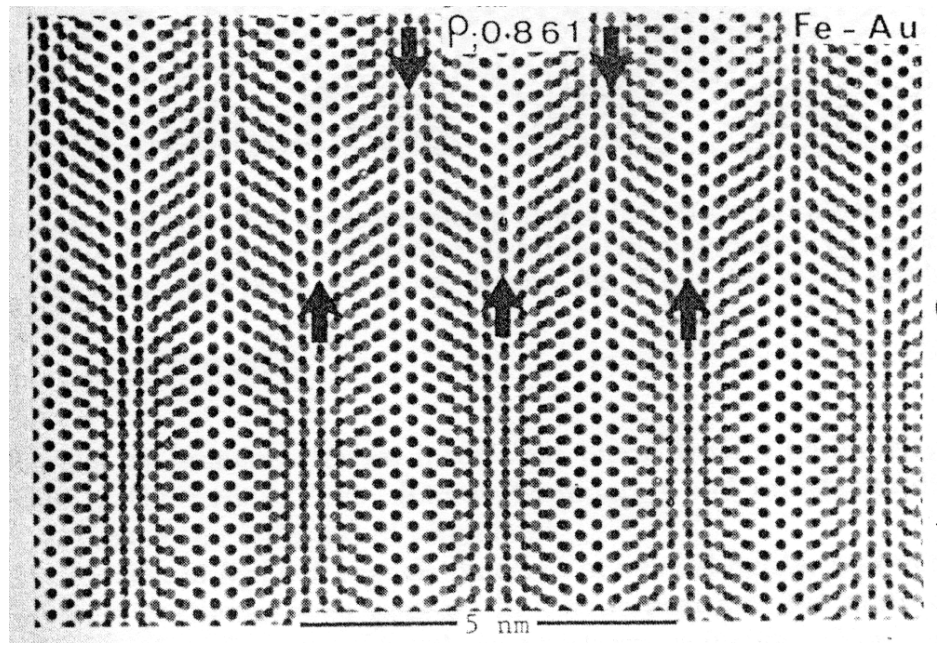

Abbildung 3.5: Überlagerung der (111)-Gold-Atompositionen mit den (110)-Eisen-Atompositionen. Es ist zu erkennen, dass die Gitter in senkrechter Richtung sehr gut passen und auch in waagerechter Richtung eine Schwebung mit einer Wellenlänge von 2,5nm vorliegt [Bruce et al., 1978]. ringer und umgekehrten Vorzeichens als im System Niob/Kupfer. Danach zeigt Eisen nur in $\langle 110\rangle$-Richtung eine größere Fehlpassung von etwa 13 Prozent (Abbildung 3.5). Die Aufklärung der tatsächlich vorhandenen Struktur der Edelmetall- und Eisenschichten ist in diesem Fall um einiges schwieriger als z.B. im System Niob/Kupfer. Aufgrund der größeren Streufaktoren und der größeren Lagendicke der Edelmetalle liefert die Röntgenbeugung nur Informationen über die Edelmetalle. Insbesondere fallen die (200)-Reflexe der Edelmetalle mit den (220)-Reflex des Eisens zusammen, so dass diese nicht unterschieden werden können (Tabelle 3.2).

Da die langwellige Rauigkeit der Schichtpakete im Eisen/Silber sehr groß sind, lassen sich dort die Überstrukturen nicht deutlich erkennen [Herweg, 1999]. Für Eisen/Gold erhält man aus der Analyse der Überstrukturen einen Gitterparameter für Gold, der fast dem Wert im Massivmaterial entspricht und erhält so einen Hinweis auf ein kohärentes Aufwachsen der Lagen. Dies kann als erster Hinweis auf eine (110)-orientiertes kubisch-raumzentriertes Eisen in den Schichtpaketen betrachtet werden.

Im hochauflösenden TEM sind die (111)-Gitterebenen sowohl von Silber, als auch von Gold deutlich zu erkennen (Abbildung 3.6). Desweiteren ist zu erkennen, dass die Orientierung der Edelmetallkörner innerhalb eines Makrokorns unverändert 


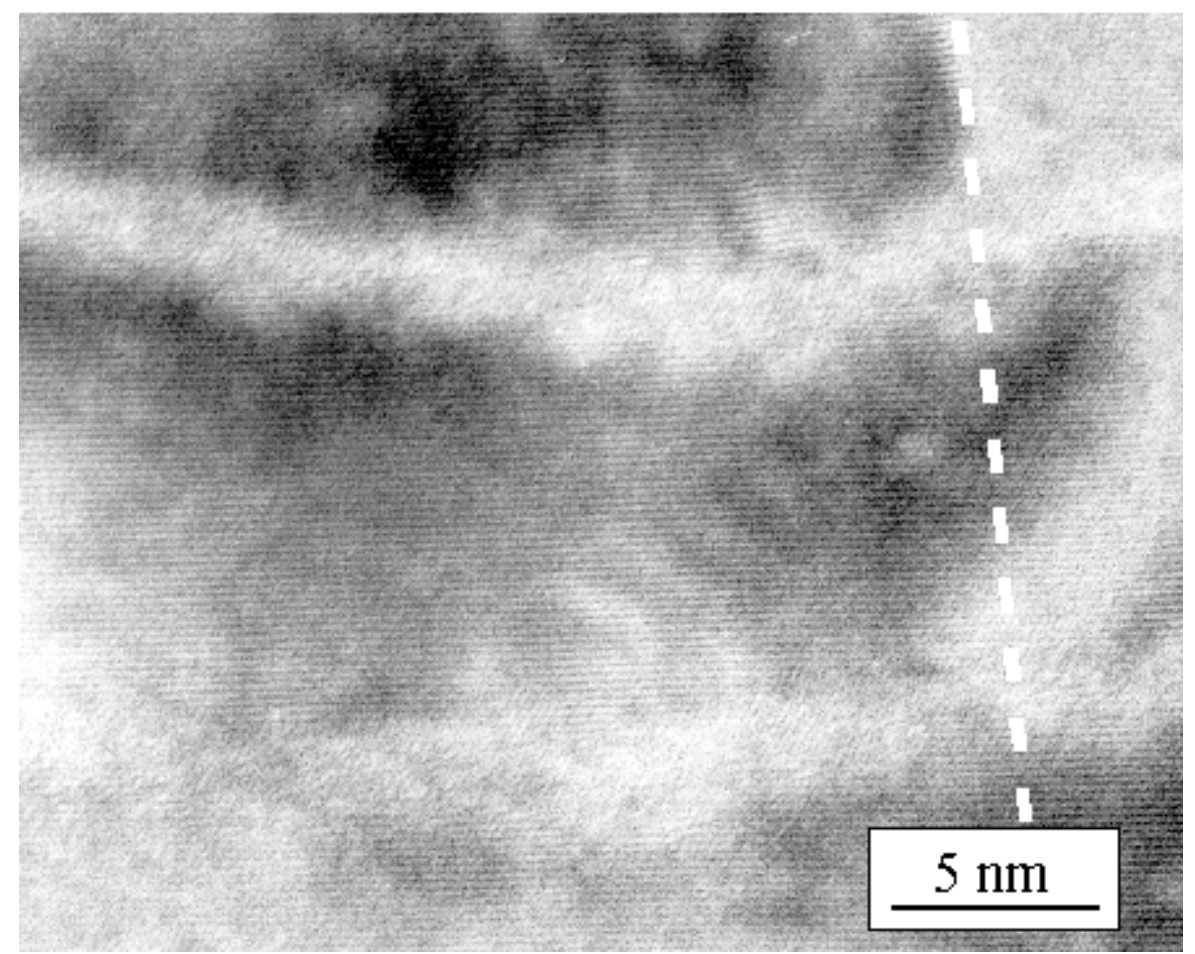

Abbildung 3.6: Fe/Ag-Vielfachschicht. Die Orientierung der (111)Ag-Netzebenen wird über die Fe-Lagen vermittelt. Der helle waagerechte Streifen ist die Fe-Lage. Senkrecht ist eine Makrokorngrenze (gestrichelte Linie) zu erkennen.

bleibt und offensichtlich durch die Eisenlage (helle waagerechte Streifen) von einer Edelmetalllage zur nächste übertragen wird. Aufgrund des Magnetismus sind keine Netzebenen des Eisens erkennbar. Auch hier gibt die präzise Übermittlung der Kristallstruktur und der Korngrenzen ein Indiz dafür, dass Eisen entsprechend der Nishiyama-Wassermann-Beziehung in (110)-Orientierung aufwachsen muss. Aus der Literatur [Bobeth et al., 1997] ist bekannt, dass dünne Eisenschichten amorph aufwachsen können, aber nur bis zu einer maximalen Schichtdicke von etwa $5 \mathrm{~nm}$. Vielfachschichten mit Eisenlagendicken von mehr als $10 \mathrm{~nm}$ sollten demnach kristallines Eisen zeigen. Dazu wurden zwei Experimente durchgeführt. Zum einen wurden Multilagen mit $10 \mathrm{~nm}$ Eisen hergestellt und im Röntgendiffraktometer untersucht. Hier zeigte sich das gleiche Bild wie schon bei den dünnen Eisenlagen, es sind keine (110)-Eisenreflexe eindeutig zu identifizieren. Zum zweiten wurden auf Kohlenstoff beschichtete TEM-Netze ein Dreilagenpaket Gold/Eisen/Gold (6 nm/0$12 \mathrm{~nm} / 6 \mathrm{~nm}$ ) abgeschieden. Diese Proben lassen sich im TEM direkt durchstrahlen. Man sieht bei sämtlichen Proben deutlich die Gitterebenen der Goldkörner. Es konnte jedoch in keiner Probe ein Moiré-Muster, das von der Überlagerung der 
Gitterebenen der interkalierten Eisenschicht und der Goldschichten her rühren könnte, gemessen werden (Abbildung 4.5, Seite 41). Lediglich die Größe der Goldkörner veränderte sich in Abhängigkeit von der Eisenlagendicke, was später noch diskutiert werden soll. In Tabelle 3.2 sind die Reflexlagen von Gold und den beiden Eisenphasen aufgetragen. Grau gekennzeichnet sind alle Goldreflexe, die mit einem kubisch-raumzentrierten Reflex des Eisens übereinstimmen. Da es keinen Reflex des kubisch-raumzentrierten Eisens gibt, der nicht von einem Goldreflex überlagert wird, lassen sich diese Eisenphase und Gold auch nicht in Beugungsbildern unterscheiden. Es wurden aber auch bei keiner der Proben Reflexe der kubisch-flächenzentrierten Eisenphase, die schon in Vielfachschichten beobachtet wurde [Störmer, 1998], gemessen . Da außer den Goldreflexen keine weiteren Reflexe zu erkennen sind und bei Eisenlagendicken von $10 \mathrm{~nm}$ eine amorphe Phase ausgeschlossen werden kann, lässt sich aus den Ergebnissen schließen, dass in den Schichtsystemen die kubisch-raumzentrierten Eisenphase gebildet wird. Um letzte Zweifel auszuschließen, wurden an den Proben zusätzlich Magnetisierungsmessun-

\begin{tabular}{|c|c||c|c||c|c||}
\hline $\boldsymbol{k f z} \boldsymbol{A u}$ & $\boldsymbol{d}_{\boldsymbol{h k l}}$ & $\boldsymbol{k r z} \boldsymbol{F e}$ & $\boldsymbol{d}_{\boldsymbol{h k l}}$ & $\boldsymbol{k f z} \boldsymbol{F e}$ & $\boldsymbol{d}_{\boldsymbol{h k l}}$ \\
\hline 111 & 1 & & & 111 & 1,12 \\
\hline 200 & 1,15 & 110 & 1,16 & 200 & 1,29 \\
\hline 220 & 1,63 & 200 & 1,64 & 220 & 1,82 \\
\hline 311 & 1,92 & & & 311 & 2,14 \\
\hline 222 & 2 & 211 & 2,01 & 222 & 2,23 \\
\hline 400 & 2,31 & 220 & 2,32 & 400 & 2,58 \\
\hline 331 & 2,52 & & & 331 & 2,81 \\
\hline 420 & 2,58 & 310 & 2,6 & 420 & 2,88 \\
\hline 422 & 2,83 & 222 & 2,84 & 422 & 3,15 \\
\hline 333 & 3 & 321 & 3,06 & 333 & 3,35 \\
\hline 440 & 3,27 & & & & \\
\hline 531 & 3,42 & & & & \\
\hline 442 & 3,46 & 330 & 3,47 & & \\
\hline
\end{tabular}

Tabelle 3.2: Radius der TEM-Beugungsringe der verschiedenen Reflexe bezogen auf den Gold-(111)Reflex. 
gen mit dem Vibrationsmagnetometer durchgeführt. Da die Sättigungsmagnetisierung des amorphen Eisens deutlich geringer als die des kubisch-raumzentrierten Eisens ist, kann auf diese Weise eine Unterscheidung vorgenommen werden. Es zeigte sich, dass die Magnetisierung sowohl der Eisen/Silber, als auch der Eisen/GoldVielfachschichten eine Magnetisierung nahe der einer Eisen Massivprobe mit einer Dicke entsprechen der Summe der Einzellagendicken zeigten. Aus der Kombination dieser Ergebnisse kann im weiteren ein Wachstum von (110)-Eisen auf (111)Gold/Silber vorausgesetzt werden.

Trotz der relativ großen Fehlpassung zwischen (110)-Eisen und (111)-Gold/Silber basierend auf der Nishiyama-Wassermann-Beziehung ist im hochauflösenden TEM ein Übertrag der Orientierung zwischen den Edelmetallschichten über das Eisen hinweg zu beobachten. Die Breite der Edelmetallkörner liegt im System Fe/Au bei $30 \mathrm{~nm}$, im System Fe/Ag bei 70-90 nm. Die unterschiedlichen Breiten sind wahrscheinlich auf die für Gold im Vergleich zu Silber fast doppelt so hohe Aufwachsrate zurückzuführen. In Wachstumsrichtung konnten keine Korngrenzen entdeckt werden, so dass eine Höhe der Körner von $10 \mathrm{~nm}$ vorausgesetzt werden kann. Diese

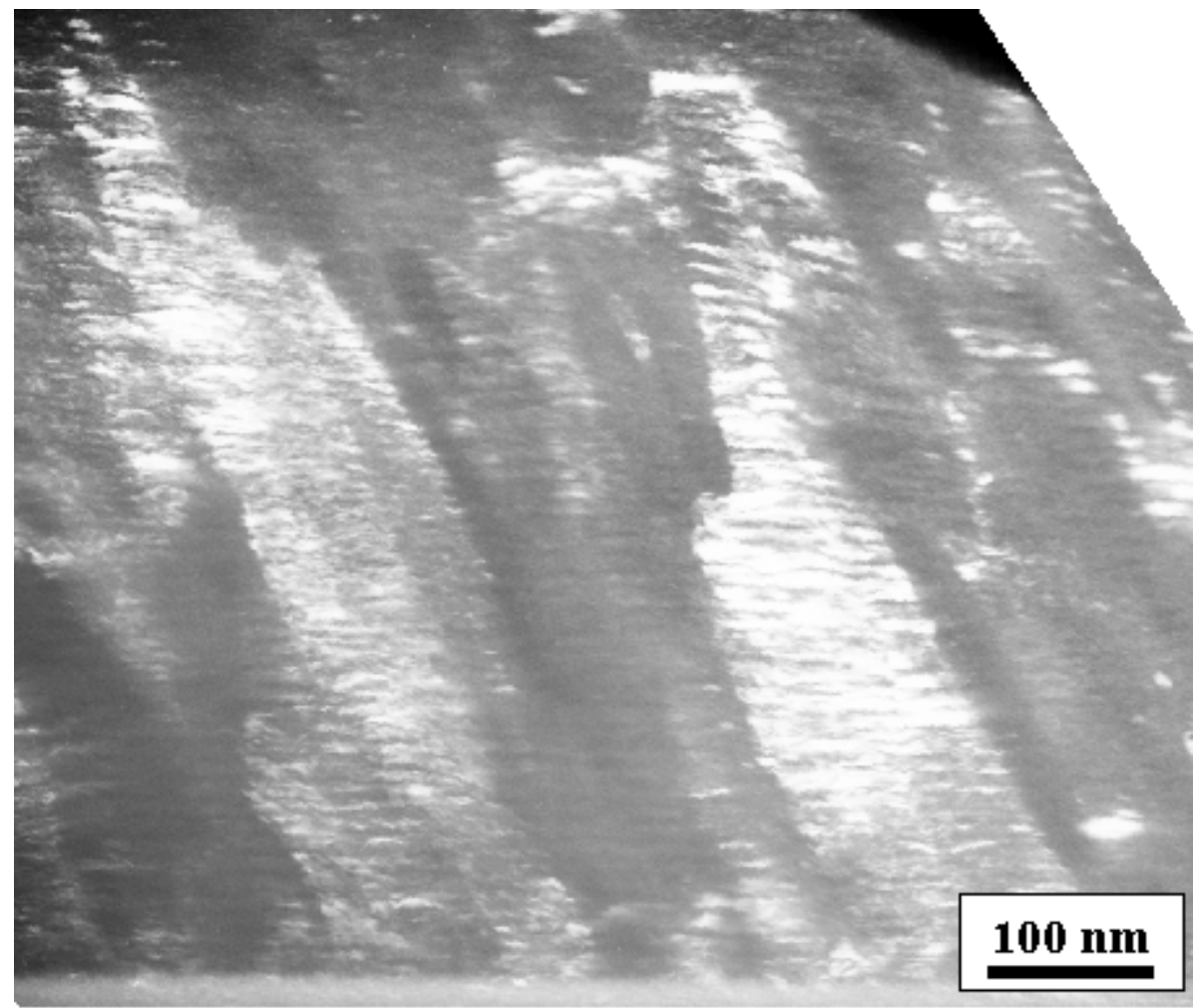

Abbildung 3.7: Dunkelfeld-Bild eines Fe/Au-ML (2 nm/10 nm). Deutlich zu erkennen die Makrokörner, die teilweise fast durch das ganze Schichtpaket wachsen. 


\subsection{Die Systeme Eisen/Silber und Eisen/Gold}

relativ großen Körner in Verbindung mit dem epitaktischen Übertrag der Orientierung erzeugt in beiden Systemen ein Säulenwachstum, bei dem innerhalb jeder Säule die Orientierung der Edelmetallkörner erhalten bleibt. Abbildung 3.7 zeigt ein Dunkelfeldbild einer solchen Eisen/GoldVielfachschicht. Hell erleuchtet sieht man Körner, die die gleiche in-plane Orientierung haben. Es ist

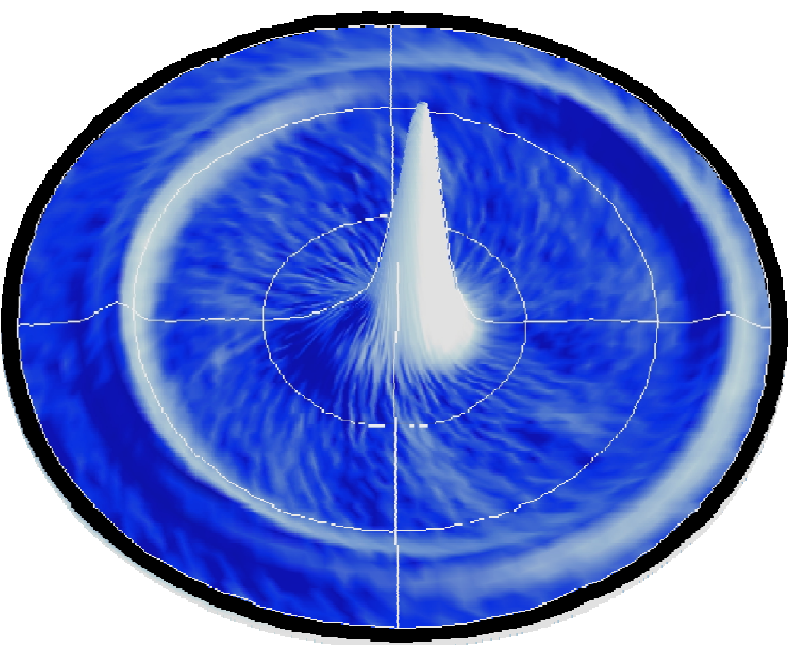

Abbildung 3.8: (111)-Drahttextur eines Fe/Au-Multilayers (2nm/10nm). Dabei ist der zentrale Peak um etwa $9^{\circ}$ in Richtung der Au-Sputterquelle geneigt. auch zu erkennen, dass diese Säulen zum Teil bis zu $1 \mu \mathrm{m}$, also fast durch das gesamte Schichtpaket wachsen. Es kommt aber auch vor, dass die Säulen vollständig oder teilweise überwachsen werden, oder dass neue Säulen gebildet werden, die schnell eine fast konstante Breite erreichen und mit dieser auch weiterwachsen. Diese Säulen werden im Folgenden als Makrokörner bezeichnet. Ähnlich wie beim System Niob/Kupfer sind die Makrokörner bzw. die Kristallstruktur aus der senkrechten gekippt, wie auch in den Texturuntersuchungen klar zu erkennen ist (Abbildung 3.8, vergleich Niob/Kupfer: Abbildung 3.3).

Vergleicht man die TEM-Bilder in Abbildung 3.9 und Abbildung 3.10 miteinander, die Eisen/Gold und Eisen/Silber Vielfachschichten mit jeweils $2 \mathrm{~nm}$ Eisen und $10 \mathrm{~nm}$ Gold bzw. Silber zeigen, fallen deutliche Unterschiede zwischen den Schichtpaketen direkt ins Auge. Vom Substrat weg wachsen beide Schichtsysteme zunächst sehr glatt auf. Nach etwa fünf Doppellagen beginnt sich in beiden Systemen eine Welligkeit der Grenzflächen aufzubauen. Die Eisen/Silber Schichten zeigen eine unregelmäßige, langwellige Rauigkeit mit einer Wellenlänge von etwa 80$100 \mathrm{~nm}$, die nur partiell durch die Korngröße der Makrokörner definiert wird. Die Krümmungsradien der Grenzflächen sind an den Minimal- und Maximalpositionen vergleichbar. Im System Eisen/Gold dagegen ist die Wellenlänge der Rauigkeit direkt mit den Korngrenzen der Makrokörner korreliert. An diesen Korngrenzen erreichen die Eisenlagen ein Minimum bezüglich ihres Abstands vom Substrat. Im Gegensatz zum System Eisen/Silber sind die Krümmungsverhältnisse hier an den 


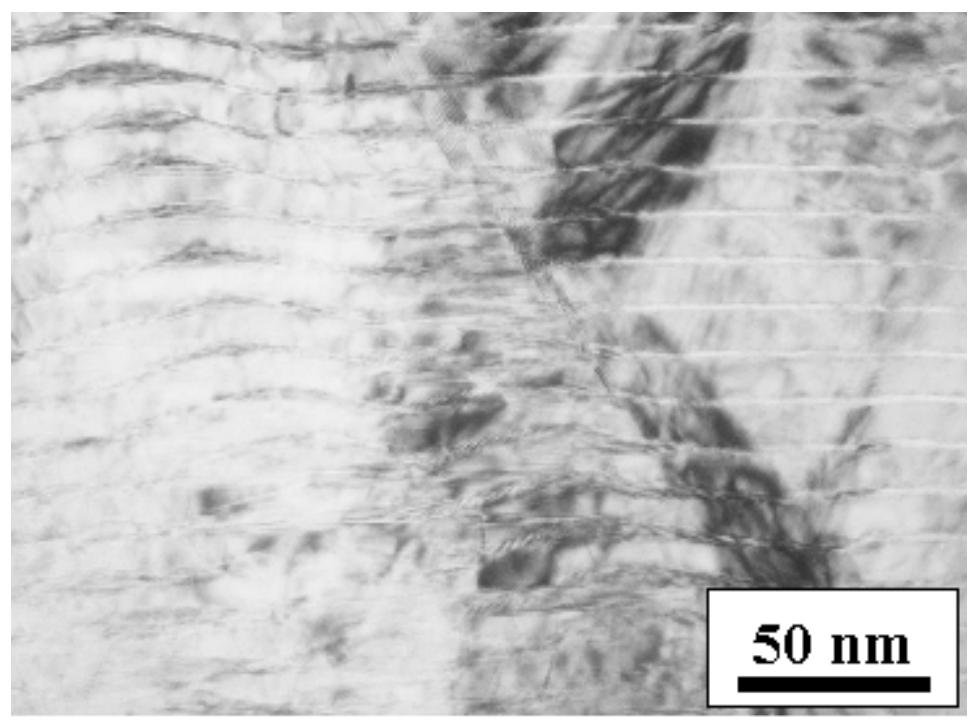

Abbildung 3.9: Fe/Ag-(2 $\mathrm{nm} / 10 \mathrm{~nm})$-Vielfachschichten, im ursprünglichen Zustand..

Minimal- und Maximalpositionen deutlich unterschiedlich. Während die Maxima breite gleichmäßige Bögen bilden, sind die Minima fast spitz zulaufend, ähnlich wie bei einer Aneinanderreihung von Halbkreisen. Die untere Seite der Eisenlage läuft dabei spitzer zu, als es die obere Seite der Lage tut.

Da die mechanischen Grenzflächenspannungen aufgrund der Gitterfehlpassung in beiden Systemen ähnlich sind, liegt die Annahme nahe, dass die unterschiedlichen Randlöslichkeiten in den Systemen zu diesem Unterschied in der Mikrostruktur führen. An den Tripelpunkten zwischen den Eisen/Edelmetall-Grenzflächen und einer Edelmetallkorngrenze bildet sich entsprechend den Grenzflächenspannungen ein

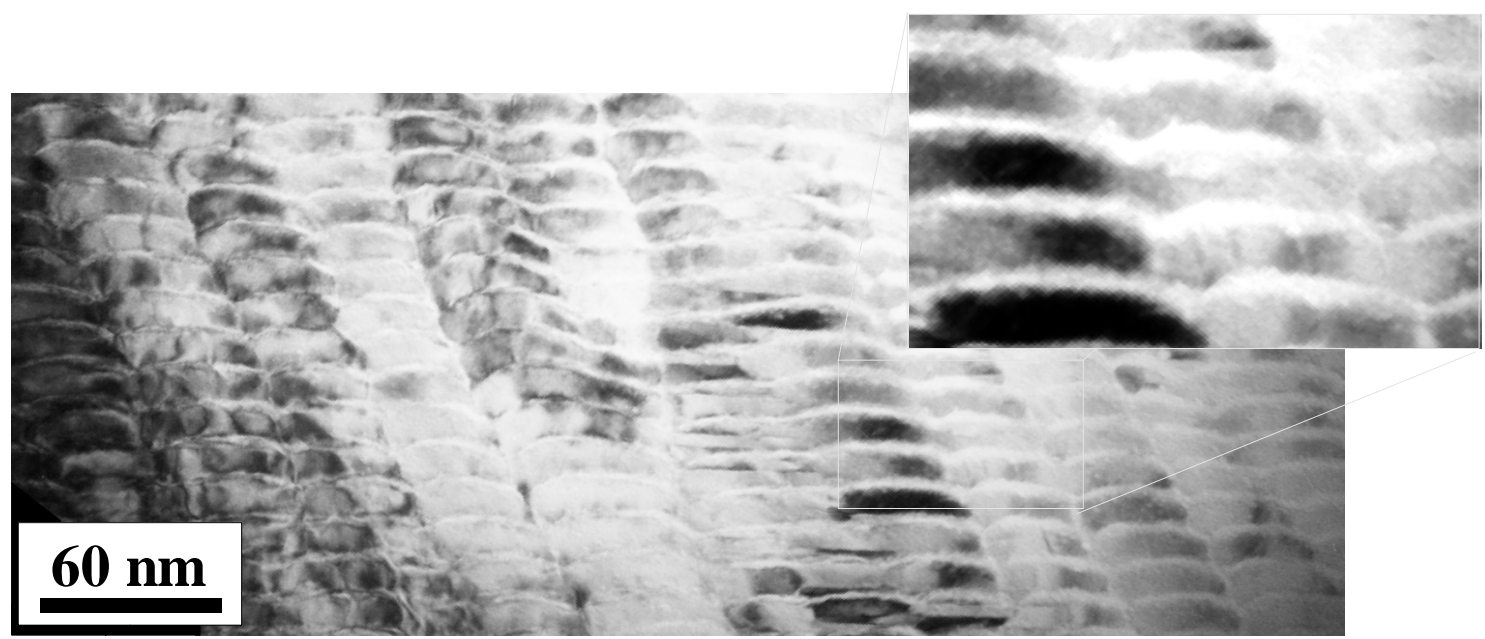

Abbildung 3.10: Fe/Au-(2 nm/10 nm)-Vielfachschichten, im unbehandelten Zustand nach der Deposition. 
Kräftegleichgewicht aus (Abbildung 3.11) [Mullins, 1957]. Danach verhalten sich die Winkel an einem solchen Tripelpunkt entsprechend folgender Formel:

$$
E_{K G}=2 E_{G F} \cos \frac{\Theta}{2}
$$

Der chemische Beitrag der Grenzflächenspannung und die Korngrenzenergie zwischen Eisen und Silber ist mit etwa $2000 \mathrm{~mJ} / \mathrm{m}^{2}$ (siehe Kapitel 6.1) ${ }^{2}$ sehr viel größer als die einer Silberkorngrenze mit $380 \mathrm{~mJ} / \mathrm{m}^{2}$ [Utigard, 1993]. Das bedeutet nach Gl. 3.1, dass die Grenzfläche nur geringe Einkerbungen mit einem Winkel $\Theta=170^{\circ}$ an den Korngrenzen des Silbers zeigt. Im System Eisen/Gold sieht das an-

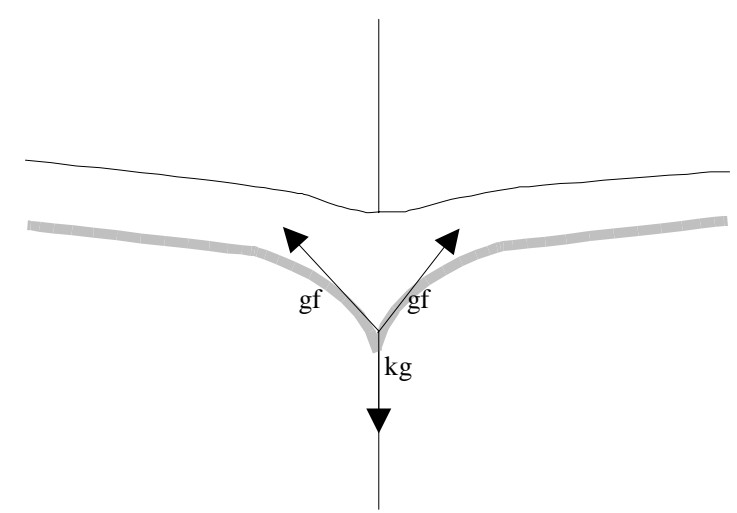
Abbildung 3.11: Grenzflächenspannung an den Tripelpunkten. Vgl. auch Ausschnitt in Abbildung 3.10. ders aus. Auch hier wird in der Nähe der Korngrenzen eine Relaxation der mechanischen Grenzflächenspannung angenommen. Beim Aufwachsen von Eisen auf Gold mischt sich etwas Eisen im Gold und bildet eine Mischphase [Labat et al., 1999], die dazu führt, dass die Grenzflächenspannung von etwa $1000 \mathrm{~mJ} / \mathrm{m}^{2}$ leicht reduziert wird. Außerdem ist an Eisen/GoldGrenzflächen auch der chemische Beitrag zur Grenzflächenenergie mit $80 \mathrm{~mJ} / \mathrm{m}^{2}$ deutlich kleiner als im System Eisen/Silber. Die Grenzflächenspannung wird daher mit etwa $1000 \mathrm{~mJ} / \mathrm{m}^{2}$ abgeschätzt (siehe Kapitel 6.1). Die Korngrenzenergie im Gold hat nach [Utigard, 1993] einen Wert von $380 \mathrm{~mJ} / \mathrm{m}^{2}$. Hier ergibt sich daher ein Winkel der Einkerbung von $\Theta=160^{\circ}$. Beim Wachstum von Gold auf Eisen bildet sich laut Phasendiagramm keine Mischphase, so dass keine Relaxation der Eisen/Gold-Grenzfläche stattfinden kann. Der Winkel an der Oberseite der Eisenschicht ist daher größer als an der Unterseite. Das Ergebnis dieses Prozesses ist auf den TEM-Bildern deutlich zu erkennen (vergrößerter Ausschnitt in Abbildung 3.10). Auf den TEM-Bildern ist auch die leichte Verdickung der Eisenlage in den Makrokorngrenzen zu erkennen. Der beschriebene Prozess ist eine Ursache für die deutlichere Struktur der Makrokörner in den Eisen/Gold-Vielfachschicht mit den Makro-

2 Dabei wurde davon ausgegangen, dass die Spannungsenergie in der Nähe der Korngrenzen relaxieren kann. 


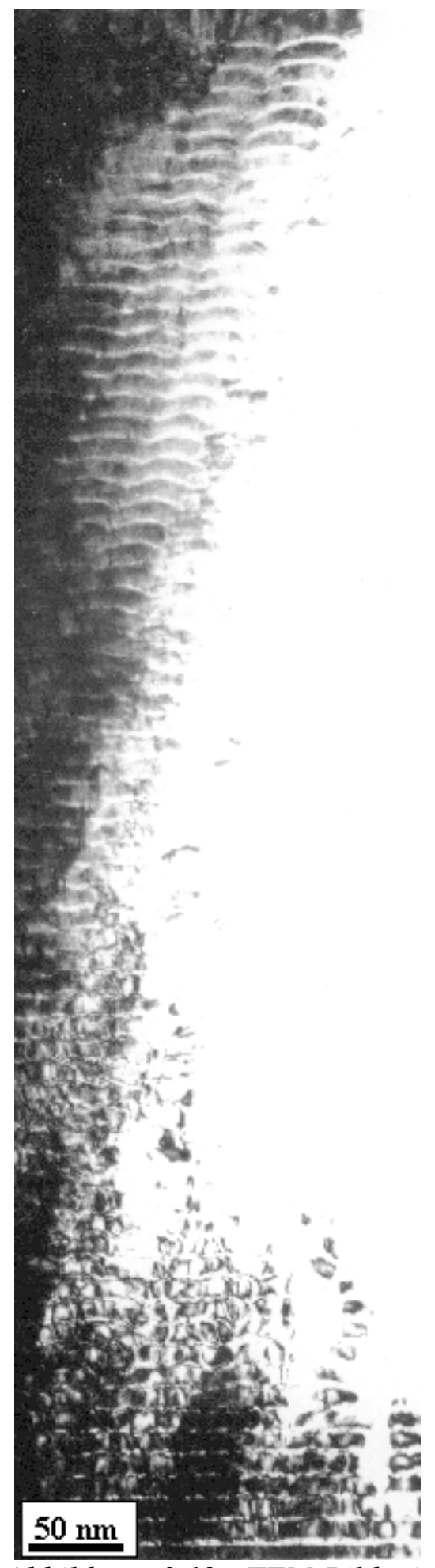

körnern und ihren bogenförmigen Grenzflächen.

Eine zweite Ursache für die Ausbildung der bogenförmigen Makrokörner ist der Abbau von Spannungen. Ein Hinweis darauf findet sich auf den TEM-Bildern. Die hell-dunkel-Kontraste nahe dem Substrat zeigen starke Spannungen im Material an (Abbildung 3.12). Je weiter die Schicht jedoch wächst, um so weniger dieser Spannungskontraste sind auf der Abbildung zu sehen. Bei genauer Betrachtung geht diese Veränderung der Spannung parallel mit der Ausbildung der gebogenen Grenzflächen an den Makrokorngrenzen vonstatten. Die Grenzflächenenergie an den durch die NishiyamaWassermann-Beziehung definierten Grenzflächen ist durch die starken mechanischen Spannungen sehr groß. Eine Abschätzung der Grenzflächenenergie wird später in Kapitel 6.2.2 vorgenommen. Durch die Ausbildung einer gebogenen Grenzfläche wird die definierte Grenzfläche gestört und die Orientierungsbeziehung zwischen dem flächenzentrierten und dem raumzentrierten Gitter geht verloren. Die Grenzfläche hat dadurch semikohärente und inkohärente Anteile, die eine sehr viel kleinere Energie haben als die glatte, kohärente Grenzfläche [Borchers, Troche et al., 2000].

Abbildung 3.12: TEM-Bild einer Fe/Au-(2 $\mathrm{nm} / 10 \mathrm{~nm})$-Vielfachschicht. Im unteren Bereich nahe dem Substrat sind Spannungskontraste zu erkennen, die mit zunehmender Rauigkeit verschwinden. 


\subsection{Vergleichssysteme Kupfer/Kobalt, Kohlenstoff/Kobalt}

\subsubsection{Kupfer/Kobalt}

Um den Einfluss der Fehlpassung auf die Desintegration untersuchen zu können, wurde anhand des Systems Kobalt/Kupfer ein Schichtsystem untersucht, das eine breite Mischungslücke hat, aber für seine kleine Grenzflächenenergie bekannt ist. Die Gitterparameter von Kobalt und Kupfer sind nahezu identisch, daher werden in diesem System kohärente Grenzflächen gefunden [Rätzke et al., 1999].

Die Mischungslücke in diesem System ist bis $420{ }^{\circ} \mathrm{C}$ ideal, bei $600^{\circ} \mathrm{C}$ löst sich etwa ein Prozent Kupfer im Kobalt (Abbildung 3.13). Aus der Symmetrie der Mischungslücke lässt sich auch die Ähnlichkeit in der Chemie der beiden Metalle erkennen.

Die Gleichgewichtsphase des Kobalt bei Raumtemperatur ist eine hexagonaldichteste Packung, die bei hohen Temperaturen in eine kubisch-flächenzentrierte Struktur übergeht. In Vielfachschichten wird jedoch häufig beobachtet [Hamp, 1997], dass auch die kubische Struktur stabilisiert wird, solange seine Lagendicke nicht zu groß wird. In den Diffraktogrammen der Kobalt/Kupfer-Vielfachschichten mit Einzellagendicken von Kobalt/Kupfer von $1 \mathrm{~nm} / 3 \mathrm{~nm}$ und $2 \mathrm{~nm} / 10 \mathrm{~nm}$ sind weder Reflexe der hexagonalen noch der kubischen Phase zu identifizieren, was wahrscheinlich auf den geringen Volumenanteil zurückzuführen ist. Die leichte Verschiebung des Kupfer-(111)-Reflexes zu größeren Winkeln deutet darauf hin, dass dieser ein gemeinsamer (111)-Reflex beider Metalle ist.

Überstrukturen waren nur in wenigen Probe messbar, was darauf hindeutet, dass die Schichten nicht sehr glatt aufwachsen. Anpassungen der Überstruktur oder des gemeinsamen Re- fatt, 1984].

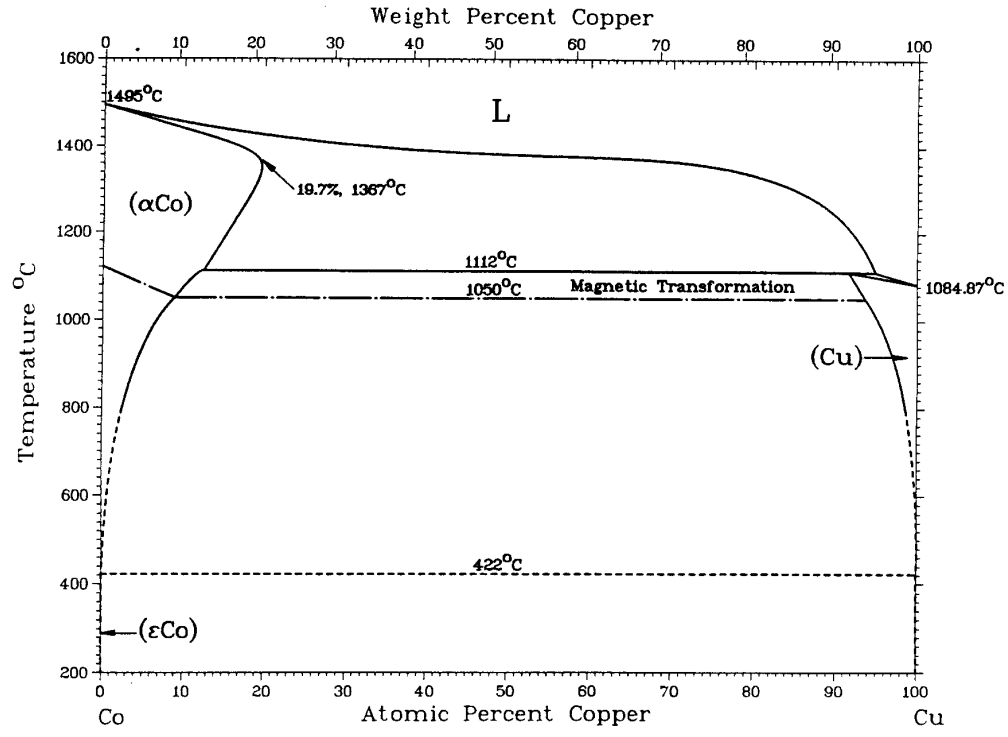

Abbildung 3.13: Phasendiagramm für Kupfer/Kobalt [Mof- 
flexes ergeben bei Vielfachschichten Kobalt/Kupfer- $(2 \mathrm{~nm} / 10 \mathrm{~nm})$ Rauigkeiten von etwa $10 \mathrm{~nm}$.

\subsubsection{Kobalt/Kohlenstoff}

In sämtlichen zuvor betrachteten Systemen liegen beide Komponenten in kristalliner Form vor. Um den Einfluss des kristallinen Gitters und dessen Korngrenzen auf die Desintegration auszumachen, wurden des Weiteren Kobalt/Kohlenstoff Vielfachschichten hergestellt. Auch in diesem System liegt eine ausgedehnte Mischungslücke bis hinauf $\mathrm{zu} 1309^{\circ} \mathrm{C}$ vor $(\mathrm{Ab}-$ bildung 3.14). Während des Sputterprozesses wächst Kohlenstoff in der Regel amorph auf [Bobeth et al., 1997]. Neben den eigenen Untersuchungen am System Kobalt/Kohlenstoff liegen zusätzlich Arbeiten anderer Gruppen an den Systemen Wolfram/Kohlenstoff und Nickel/Kohlenstoff vor, die ebenfalls auf ihre thermische Stabilität hin untersucht wurden und im Folgenden als Vergleichsysteme dienen .

Es wurden Schichten mit $2 \mathrm{~nm}$ Kobalt und 4 bzw. $10 \mathrm{~nm}$ Kohlenstoff hergestellt. Im Klein-

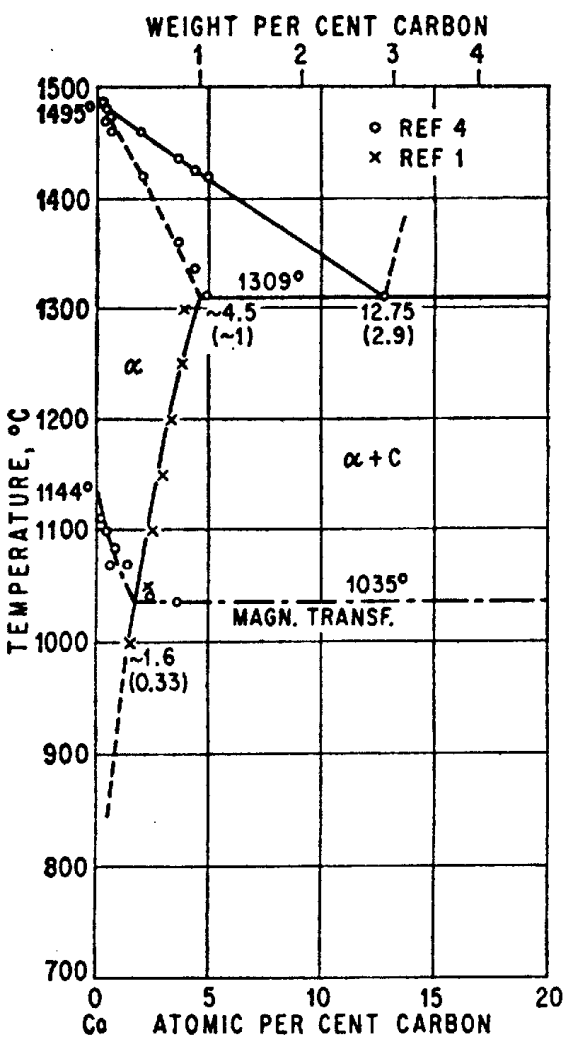

Abbildung 3.14: Phasendiagramm Kohlenstoff/Kobalt. Es gibt nur eine messbare Löslichkeit von Kohlenstoff in Kobalt [Moffatt, 1984]. winkelbereich des Diffraktogramms lassen sich bis zu drei Maxima erkennen (Abbildung 3.15). Aufgrund dieser Maxima lässt sich nicht nur feststellen, dass die Schichten sehr glatt aufgewachsen sind, sondern es lässt sich auch die Periodenlänge des Schichtsystems sehr genau messen. Das in Abbildung 3.16 gezeigte Schichtsystem hat nach der Messung aus Abbildung 3.15 danach eine Periodenlänge von 14,6 nm. Die Lagen sind demnach etwas dicker geworden, als mit $2 \mathrm{~nm}$ Kobalt und $10 \mathrm{~nm}$ Kohlenstoff geplant war. Die in Abbildung 3.16 auffällige Veränderung der Dickenverhältnisse der Lagen hängt mit der Abbildungsmethode zusammen. Um den Kontrast wurde die Probe defokussiert, 


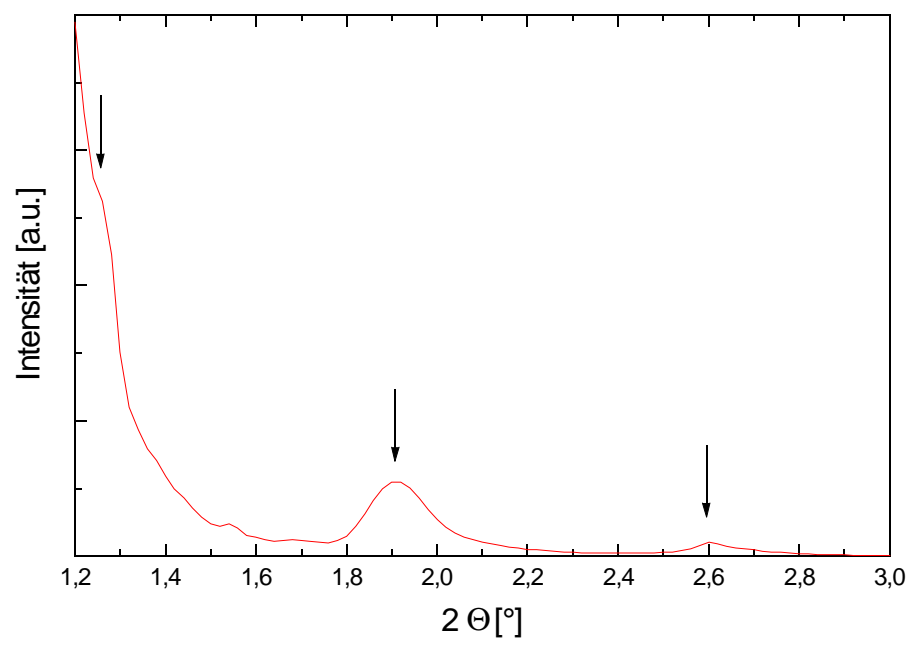

Abbildung 3.15: Kleinwinkel Überstruktur in Kobalt/Kohlenstoff Vielfachschichten. Das Auftreten mehrerer Reflexe zeugt von sehr glatten Grenzflächen.

was die Abmessungen verzerrt. Gut zu erkennen ist aber, dass die Schichten sehr glatt aufgewachsen sind. Bereiche, die in Substratnähe eine Rauigkeit zeigen, die im weiteren Wachstum wieder verschwindet, sind vermutlich bei der Dünnung der Probe leicht beschädigt worden.

Im Gegensatz zum System Kobalt/Kupfer wächst das Kobalt hier in seiner hexagonalen Phase auf. Im Diffraktogramm ist in manchen Proben der hexagonale Co-(100)-Reflex schwach zu erkennen.

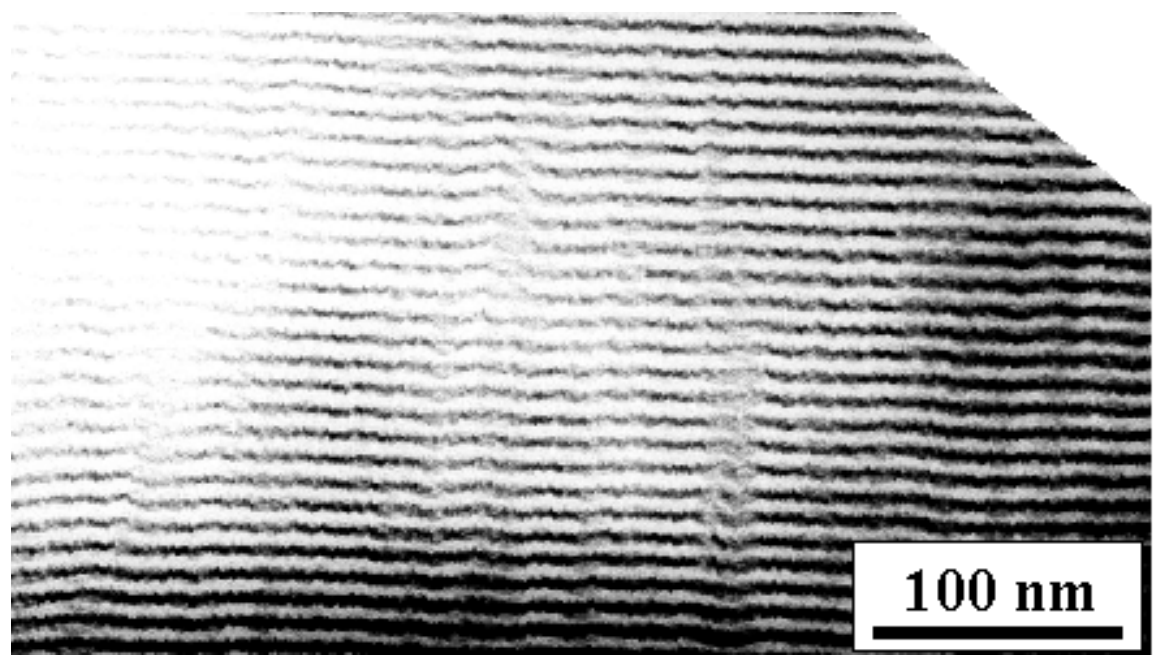

Abbildung 3.16: Kobalt/Kohlenstoff-(2 $\mathrm{nm} / 10 \mathrm{~nm})$-Vielfachschicht. Die veränderlichen Dickenverhältnisse kommen durch die Defokussierung im TEM zustande. 


\section{4 Übersicht über die wie-hergestellten Proben}

Zusammenfassend ergibt sich folgendes Bild für die wie-hergestellten Proben. Es zeigt sich, dass die Grenzflächenenergie für die Mikrostruktur der Systeme von großer Bedeutung ist. Im System Niob/Kupfer ist dieser Einfluss am wenigsten ausgeprägt. Hier findet ein Wachstum der Lagen mit inkohärenten Grenzflächen statt. Die kohärenten Grenzflächen führen beim System Kobalt/Kupfer zur Stabilisierung der metastabilen kubisch-flächenzentrierten Phase des Kobalt. In den kohärent aufwachsenden Systemen Eisen/Gold und Eisen/Silber führt die Reduktion der Grenzflächenenergie zur Bildung von Makrokörnern mit gebogenen Grenzflächen. Die Breite der Makrokörner liegt im System Eisen/Gold bei 30 nm, im System Eisen/Silber bei 70-90 nm. Aufgrund der Krümmung kann die Grenzflächenenergie durch Bildung lokal semi- bzw. inkohärenter Grenzflächen in der Nähe der Makrokorngrenzen erheblich reduziert werden. Diese Reduktion der Grenzflächenenergie überwiegt die Energie zur Vergrößerung der Grenzfläche bei weitem.

Im System Kobalt/Kohlenstoff gibt es weder eine Orientierungsbeziehung zwischen den Gittern, noch Mischkristallbildung oder Korngrenzen. Für eine minimale, d.h. glatte Grenzfläche ist auch die Grenzflächenenergie minimal. Die Schichten wachsen glatt auf. 


\section{Die Mikrostruktur während und nach der Auslagerung}

Um neben dem Endzustand der Vielfachschicht nach der Auslagerung auch den Übergang zu dieser Mikrostruktur nachvollziehen zu können, wurden Proben im Röntgendiffraktometer ausgelagert. So ist es möglich, in kurzen Zeitintervallen von 10 Minuten, Messungen der Proben während der Wärmebehandlung durchzuführen. Zur Abbildung der Mikrostruktur nach der Auslagerung wurden zusätzlich Proben im UHV ausgelagert und anschließend im Transmissionselektronenmikroskop (TEM) und im Röntgenmikroskop (XRM) untersucht.

Um die Unterschiede aber auch die Ähnlichkeiten der verschiedenen Systeme darzustellen, werden im ersten Teil dieses Kapitels die charakteristischen Veränderungen der Systeme Niob/Kupfer, Eisen/Gold und Eisen/Silber untersucht, die alle eine Desintegration bei Temperaturen deutlich unter $600^{\circ} \mathrm{C}$ zeigen. $\mathrm{Im}$ zweiten $\mathrm{Ab}$ schnitt wird auf die Systeme Kobalt/Kupfer und Kobalt/Kohlenstoff eingegangen, die bis zu hohen Temperaturen eine stabile Lagenstruktur aufweisen.

\subsection{Desintegration in den Systemen Niob/Kupfer, Eisen/Gold und Eisen/Silber}

\subsubsection{Röntgenbeugungsuntersuchungen}

Mit Hilfe der Röntgenbeugung war es für die Systeme Niob/Kupfer und Eisen/Gold möglich, die Desintegrationstemperaturen und die Partikelbildung an Vielfachschichten mit verschiedenen Schichtzusammensetzungen sehr genau zu analysieren. Dabei stellt die Veränderung der Überstruktur eine sehr empfindliche Sonde für den Zustand der Vielfachschichten dar. Aufgrund des Fehlens von Überstrukturen im System Eisen/Silber war die Analyse der Zerfallstemperatur in Abhängigkeit von der Dicke der interkalierten Lagen nicht möglich.

In Abbildung 4.1 ist die Veränderung eines Diffraktogramms eines Eisen/Gold$\left(2 \mathrm{~nm} / 10 \mathrm{~nm}\right.$ )-Schichtpaketes während einer Auslagerung bei $330^{\circ} \mathrm{C} \mathrm{zu}$ sehen. Während die Gitterparameter im wie-hergestellten Zustand durch eine Anpassung des Stufenmodells an die Messung erhalten werden, können sie nach der Desintegration direkt aus der Lage der entsprechenden Reflexe gewonnen werden. Es zeigte sich, dass die Desintegration in den Systemen Niob/Kupfer und Eisen/Gold 


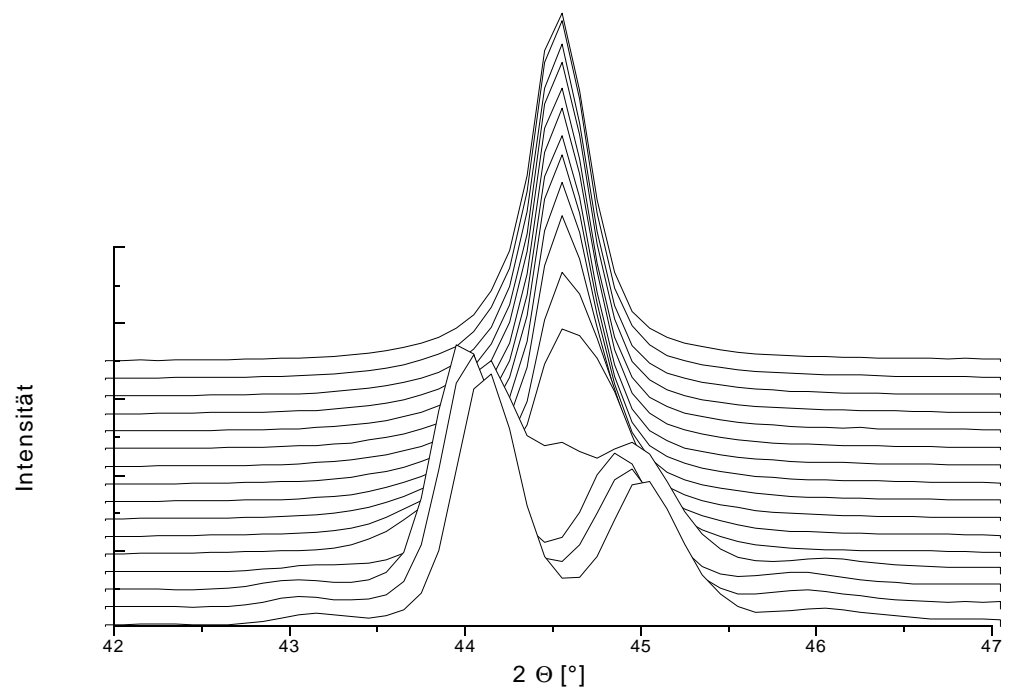

Abbildung 4.1: Veränderung der Überstruktur einer Eisen/Gold.(2 nm/10 nm)-Vielfachschicht bei $330^{\circ} \mathrm{C}$, gemessen alle 140 Sekunden.

gleichzeitig mit der Relaxation der inneren Spannungen der Komponenten einhergeht. Dazu ist in Abbildung 4.2 eine Messung an einer Niob/Kupfer-(4 nm/4 nm)Vielfachschicht gezeigt. Beide Metalle relaxieren bei etwa $400^{\circ} \mathrm{C}$, während die Überstruktur zeitgleich verschwindet. Da im System Eisen/Gold keine Eisenreflexe erkennbar waren, ist dort nur die Relaxation des Goldes während des Verschwindens der Überstruktur messbar.

Im System Eisen/Silber relaxieren die Spannungen nicht bei gleicher Temperatur. Bei Schichtsystemen mit $2 \mathrm{~nm}$ Eisen und $10 \mathrm{~nm}$ Silber zeigte sich zuerst eine Relaxation der Silberlagen bei etwa $250^{\circ} \mathrm{C}$, während die Eisenlagen erst oberhalb von $400^{\circ} \mathrm{C}$ relaxierten [Herweg, 1999]. Bei der Relaxation des Silbers handelt es sich vermutlich um frühe Erholungsprozesse in Verbindung mit der Einführung weiterer Zwillingsgrenzen. Dabei kann nicht ausgeschlossen werden, dass eine erste Teilrelaxation des Eisens während der Relaxation des Silbers stattfindet. Wie später noch ausgeführt wird, bleiben die Vielfachschichten während der Relaxation des Silbers stabil und zerfallen erst während der endgültigen Relaxation des Eisens. 


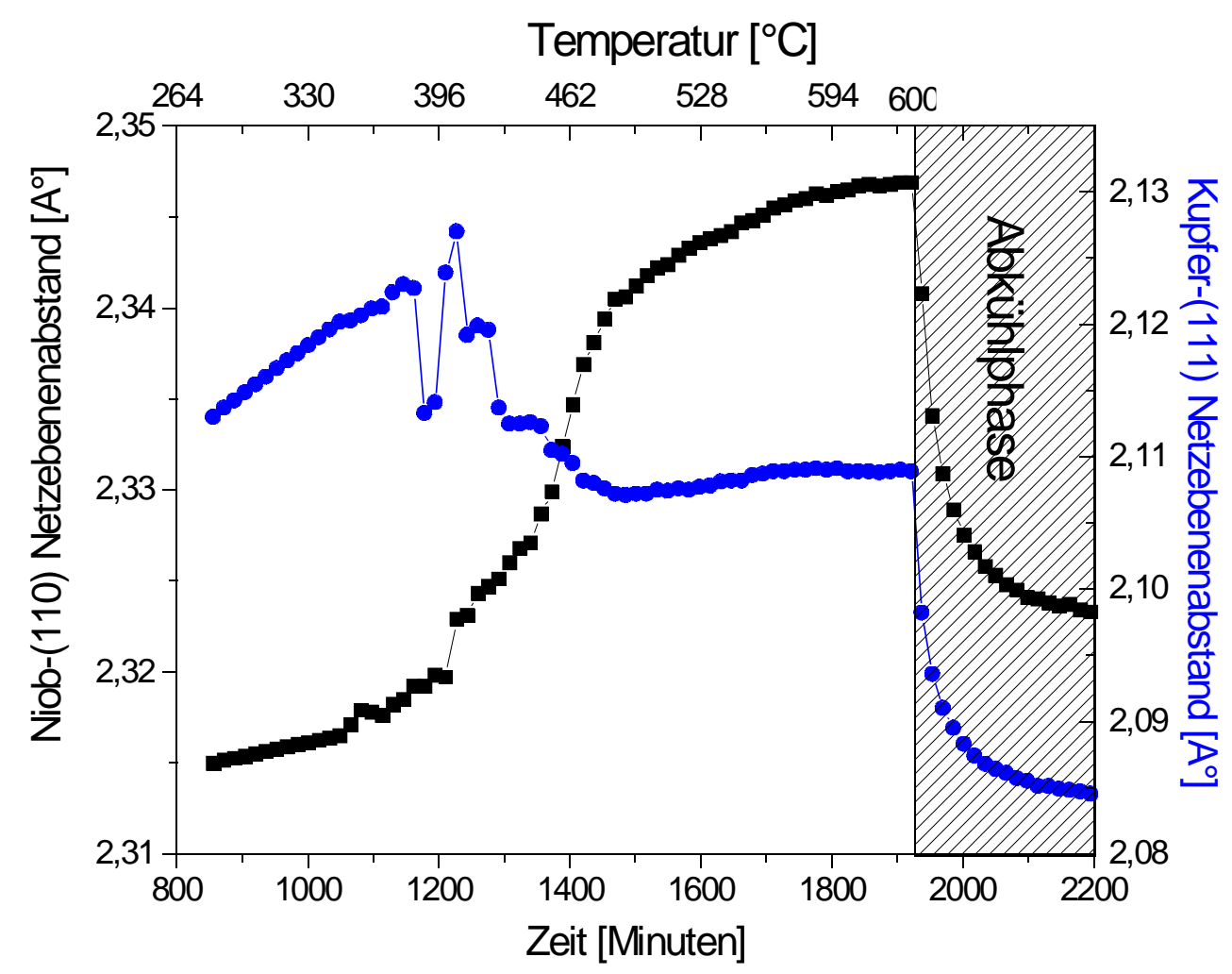

Abbildung 4.2: Veränderung der Netzebenenabstände eines Niob/Kupfer$(4 \mathrm{~nm} / 4 \mathrm{~nm})$-Schichtpaketes während der Auslagerung. Es ist eine zeitgleiche Relaxation der Metalle während der Desintegration erkennbar .

Aufgrund dieser Messungen lässt sich die Aussage treffen, dass die Desintegration der Schichtpakete durch die Relaxation der interkalierten Komponente angezeigt wird. Diese muss jedoch nicht mit der Relaxation des Matrixmetalls übereinstimmen. Auf diese Weise ist neben der Veränderung der Überstruktur eine weitere Möglichkeit der Bestimmung der Desintegrationstemperatur gegeben.

Die Kinetik der Desintegration und des Partikelwachstums wurde bereits am System Niob/Kupfer untersucht [Troche et al., 1999]. Die wichtigsten Ergebnisse dieser Untersuchungen lassen sich aus Abbildung 4.3 ableiten. Dort sind isotherme Messungen der Niobpartikelgröße in Abhängigkeit von der Zeit dargestellt. Alle untersuchten Schichten enthielten die gleiche Kupferlagendicke von $4 \mathrm{~nm}$. Ein Schichtpaket mit $4,5 \mathrm{~nm}$ dicken Nioblagen blieb bei einer Auslagerung bei $600^{\circ} \mathrm{C}$ für eine Stunde stabil und zeigte dann eine schnelle Desintegration, bei der die Partikelgrößen innerhalb weniger Minuten den sechsfachen Wert der Lagendicken er- 


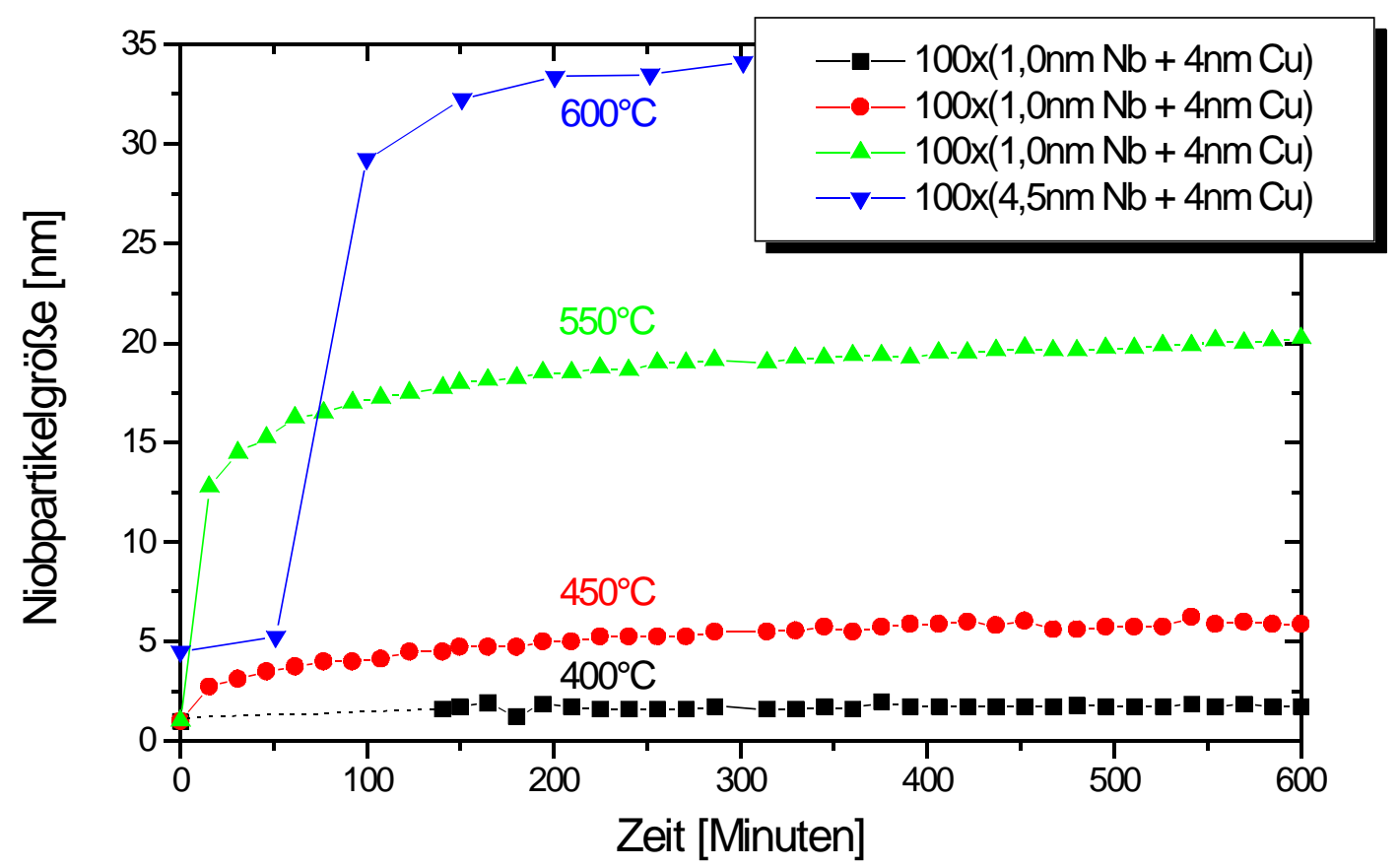

Abbildung 4.3: Abhängigkeit der zeitlichen Entwicklung der Partikelgröße von der Desintegrationstemperatur und der Lagendicke der interkalierten Lagen [Troche, 1996]

reichten. Betrachtet man dagegen die Desintegration der $1 \mathrm{~nm}$ dicken Nioblagen, so findet man einen sofortigen Zerfall. Die Desintegrationstemperatur ist also stark von der Dicke der interkalierten Schicht abhängig. Das zweite wichtige Ergebnis ist die Skalierung der Partikelgröße mit der Auslagerungstemperatur. Es ist zu erkennen, dass das anfängliche schnelle Wachstum temperaturabhängig ist. Das weitere Wachstum gehorcht einem logarithmischen Gesetz und spielt im Vergleich dazu eine untergeordnete Rolle. Eine Annäherung der Kurven an einen gemeinsamen Endwert findet nicht statt. Entsprechende Ergebnisse fanden sich auch an den Systemen Eisen/Gold und Eisen/Silber. Die Partikelgröße im nanostrukturierten Material lässt sich demnach bei den untersuchten Systemen über die Auslagerungstemperatur einstellen.

Die Abhängigkeit der Desintegrationstemperatur von der Dicke der interkalierten Schicht, die in Abbildung $4.3 \mathrm{zu}$ erkennen ist, wurde für die Systeme Niob/Kupfer und Eisen/Gold näher untersucht. Dazu wurden Vielfachschichten mit jeweils $10 \mathrm{~nm}$ Gold bzw. Kupfer mit unterschiedlich dicken Eisen- bzw. Nioblagen kombiniert. Die Ergebnisse dieser Experimente sind in Abbildung 4.4 zusammengestellt. In 


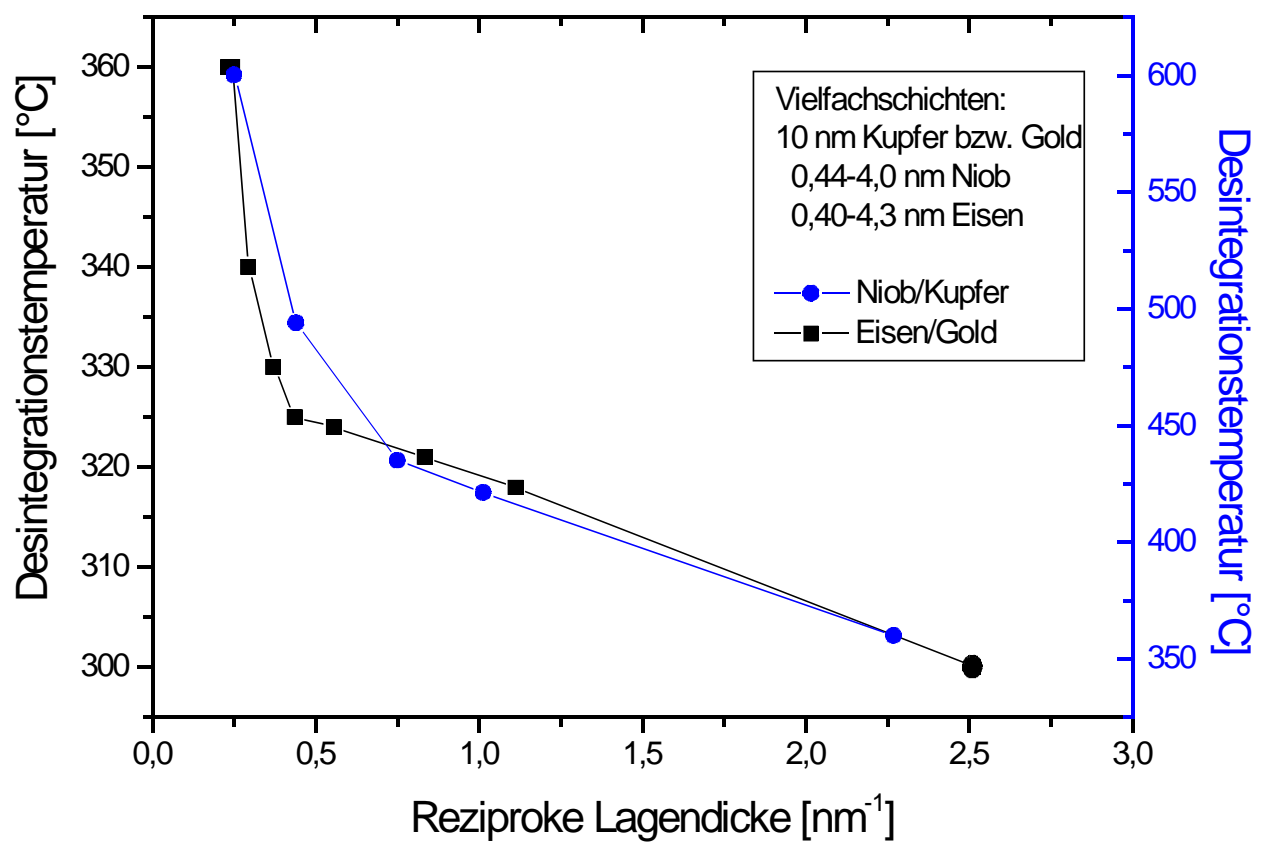

Abbildung 4.4: Desintegrationstemperaturen der Systeme Niob/Kupfer und Eisen/Gold in Abhängigkeit von der Lagendicke der interkalierten Lagen.

dieser Abbildung wurde die Desintegrationstemperatur gegen die reziproke Lagendicke der interkalierten Lage aufgetragen. Es konnte so in beiden Systemen gezeigt werden, dass die Veränderung der Desintegrationstemperatur bezüglich der Lagendicken in zwei Bereiche unterteilt ist, die unterschiedlichen Gesetzmäßigkeiten gehorchen. Für dünne Niob- bzw. Eisenlagen findet man eine reziproke Abhängigkeit der Desintegrationstemperatur von der Lagendicke. Die kritische Lagendicke beträgt im System Eisen/Gold 2,5 nm und im System Niob/Kupfer 1,5 nm. Oberhalb dieser kritischen Werte steigt die Desintegrationstemperatur mit zunehmender Lagendicke deutlich stärker an. Die Desintegrationstemperaturen im System Niob/Kupfer liegen für alle Lagendicken deutlich oberhalb der entsprechenden Werte des Systems Eisen/Gold. So desintegriert ein Eisen/Gold- $(1 \mathrm{~nm} / 10 \mathrm{~nm})$ Schichtpaket bei unter $320^{\circ} \mathrm{C}$. Beim System Niob/Kupfer muss man für ein entsprechendes Schichtpaket eine um $100^{\circ} \mathrm{C}$ höhere Temperatur wählen. Auch die Zunahme der Desintegrationstemperatur mit zunehmender Lagendicke ist im System Niob/Kupfer deutlich stärker (man beachte die Skalierung der Ordinaten). Die Desintegrationstemperatur im System Eisen/Silber konnte aufgrund der fehlenden 
Überstruktur nicht in Abhängigkeit von der Eisenlagendicke gemessen werden. Vom Absolutwert ist sie wohl eher etwas unterhalb der Desintegrationstemperatur im System Niob/Kupfer einzuordnen. Für eine Eisen/Silber- $(2 \mathrm{~nm} / 10 \mathrm{~nm})$-Schicht wurde aufgrund der Relaxationstemperatur des Eisens eine Desintegrationstemperatur von etwa $420^{\circ} \mathrm{C}$ ermittelt. Eisen/Gold-Schichten entsprechender Zusammensetzung zerfallen etwa bei $325^{\circ} \mathrm{C}$ und Niob/Kupfer-Schichten bei $480^{\circ} \mathrm{C}$. Nach diesen Ergebnissen lässt sich schließen, dass die Systeme Niob/Kupfer und Eisen/Gold trotz ihrer unterschiedlichen Mikrostruktur starke Ähnlichkeiten in Bezug auf ihre
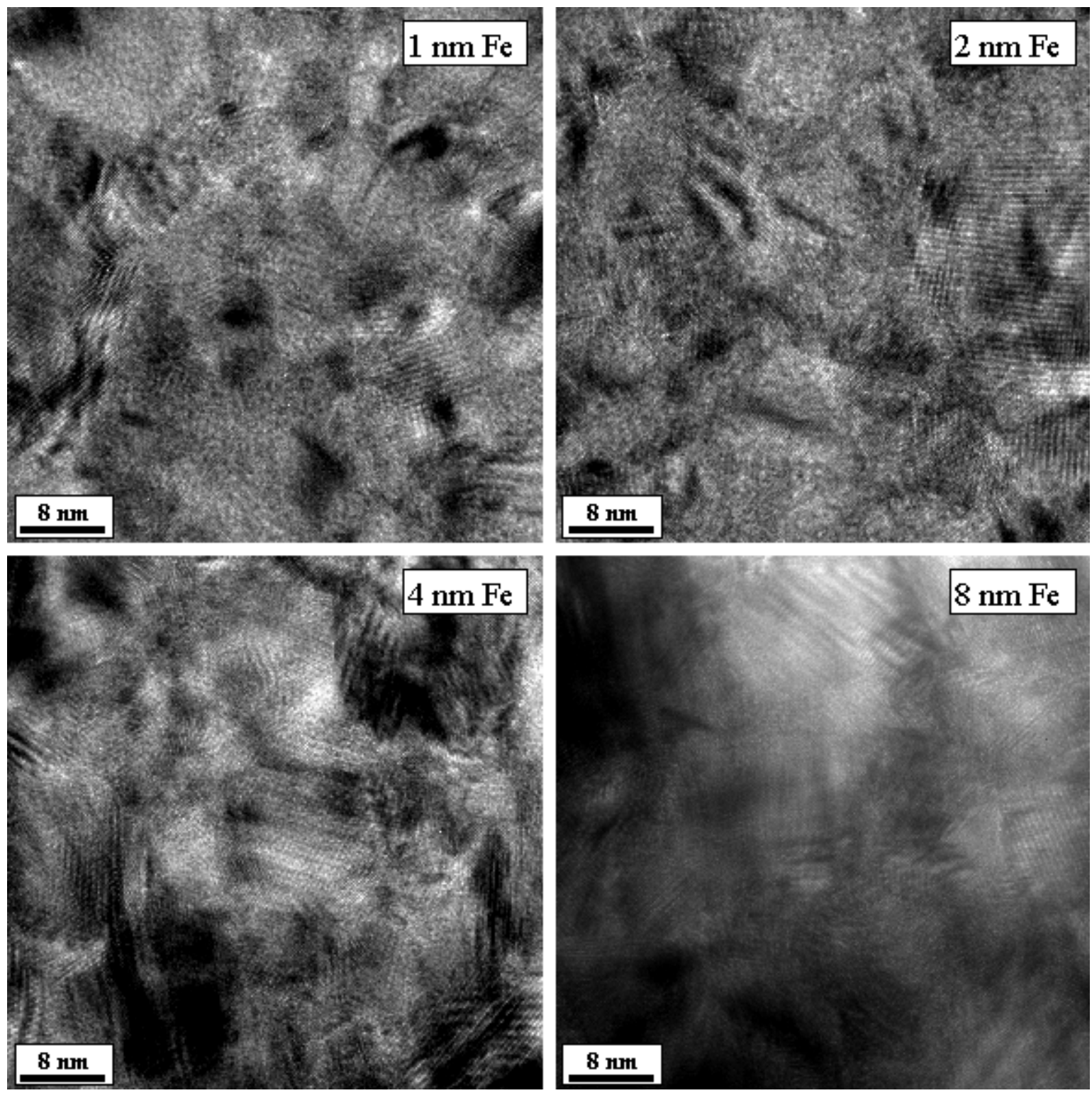

Abbildung 4.5: TEM-Durchstrahlungsbilder von Dreifachschichten Gold/Eisen/Gold. Die Goldlagen sind konstant $8 \mathrm{~nm}$ dick, die Eisenlagendicken variieren zwischen $1 \mathrm{~nm}$ und $8 \mathrm{~nm}$ (siehe Bildbeschriftung). Oberhalb von $2 \mathrm{~nm}$ Eiselagendicke schlägt die Goldkorngröße zu deutlich größeren Körnern um. 
Abhängigkeit der Desintegrationstemperatur von der Lagendicke haben. Das System Eisen/Silber unterscheidet sich trotz der ähnlichen Mikrostruktur deutlich vom System Eisen/Gold. Eine mögliche Erklärung für diesen Unterschied ist an dieser Stelle die frühzeitige Relaxation der Silberlagen. Im Rahmen der Diskussion der treibenden Kräfte wird auf die Unterschiede nochmals vertieft eingegangen.

Da zu erwarten ist, dass die deutliche Veränderung der Abhängigkeit der Desintegrationstemperatur von der Lagendicke mit der Mikrostruktur der Proben im Zusammenhang steht, wurden Eisen/Gold- $(5 \mathrm{~nm} / \mathrm{x} \mathrm{nm} / 5 \mathrm{~nm})$-Dreifachlagen direkt auf TEM-Netze deponiert, sodass die Proben im TEM ohne weitere Behandlung durchstrahlt werden konnten. Die Ergebnisse dieser Untersuchungen sind in Abbildung 4.5 für verschiedene Eisenlagendicken gezeigt. Anhand der Netzebenen sind die lateralen Korngrößen gut zu erkennen. Es ist darauf hinzuweisen, dass alle Netzebenen von Goldkörnern stammen. Vom Eisen sind weder Netzebenen noch Moirémuster sichtbar. Man erkennt, dass für Eisenlagendicken zwischen 0 und $2 \mathrm{~nm}$ Goldkörner mit einer lateralen Ausdehnung zwischen 10 und $12 \mathrm{~nm}$ wachsen. Bei Vergrößerung der Eisenlagendicken auf $4 \mathrm{~nm}$ und darüber verändert sich die Mikrostruktur deutlich. Die Korngröße der Goldkörner ist nun wesentlich ungleichmäßiger, es bilden sich viele größere Körner mit Breiten bis $25 \mathrm{~nm}$ und teilweise auch kleine Körner, die die Lücken zwischen den großen füllen. In dem Dickenbereich, in dem die Veränderung der Desintegrationstemperatur gefunden wurde, verändert sich also auch die Mikrostruktur der Schichtpakete wesentlich.

\subsubsection{Röntgenmikroskopische Untersuchungen}

Nach dieser Analyse der Desintegrationsparameter kann die Abbildung der Mikrostruktur wichtige Aufschlüsse über den Desintegrationsprozess geben. Im Röntgenmikroskop wurden dazu je eine Niob/Kupfer-( 3 x $4 \mathrm{~nm} / 4 \mathrm{~nm})$ und $(10 \times 4 \mathrm{~nm} / 4 \mathrm{~nm})$-Vielfachschicht untersucht. Diese Schichten wurde eine Stunde bei $600^{\circ} \mathrm{C}$ ausgelagert. Desweiteren wurden eine Eisen/Gold- $(10$ x $2 \mathrm{~nm} / 10 \mathrm{~nm})$ Vielfachschicht nach einstündiger Auslagerung bei $350^{\circ} \mathrm{C}$ und eine Eisen/Silber-(10 x $2 \mathrm{~nm} / 10 \mathrm{~nm})$-Vielfachschicht nach einstündiger Auslagerung bei $500^{\circ} \mathrm{C}$ untersucht. Die Schichtpakete wurden jeweils auf beiden Seiten mit einer $50 \mathrm{~nm}$ dicken Edelmetallschicht passiviert. 
Im Gegensatz zu den unbehandelten Proben dieser Typen, die kein Kontrast im Röntgenmikroskop zeigten, ließen die Proben nach der Auslagerung deutliche Kontraste erkennen. Lediglich das Niob/Kupfer-(10 x 4 nm/4 nm)-Schichtpaket, zeigte

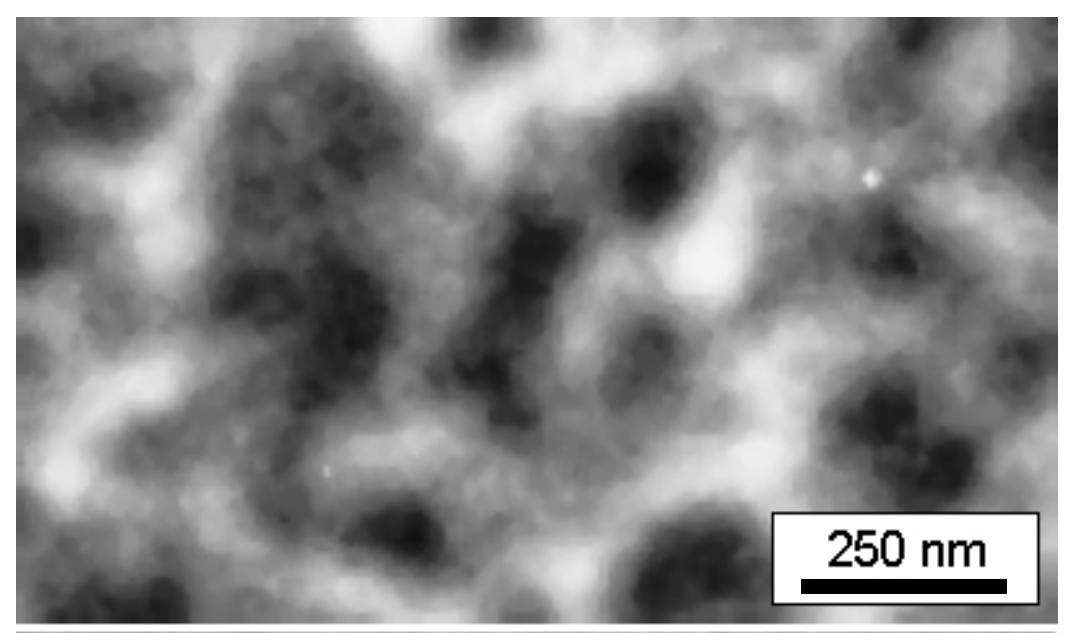

$\mathrm{Nb} / \mathrm{Cu}$ :

$3 \mathrm{x} 4 \mathrm{~nm} / 4 \mathrm{~nm}$

1h bei $600^{\circ} \mathrm{C}$

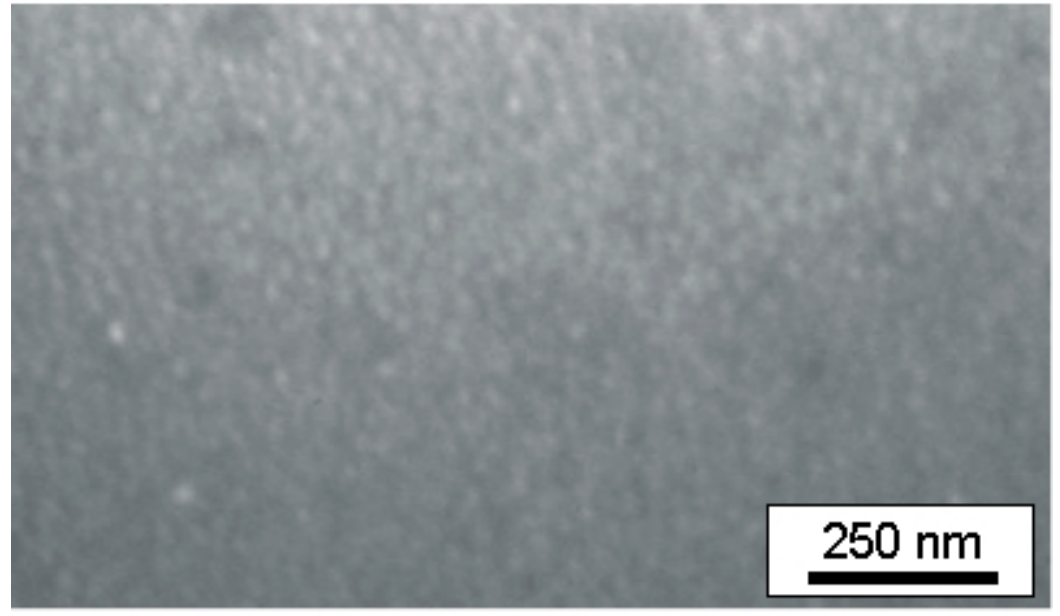

$\mathrm{Fe} / \mathrm{Au}:$

$10 \times 2 \mathrm{~nm} / 10 \mathrm{~nm}$

$1 \mathrm{~h}$ bei $350^{\circ} \mathrm{C}$

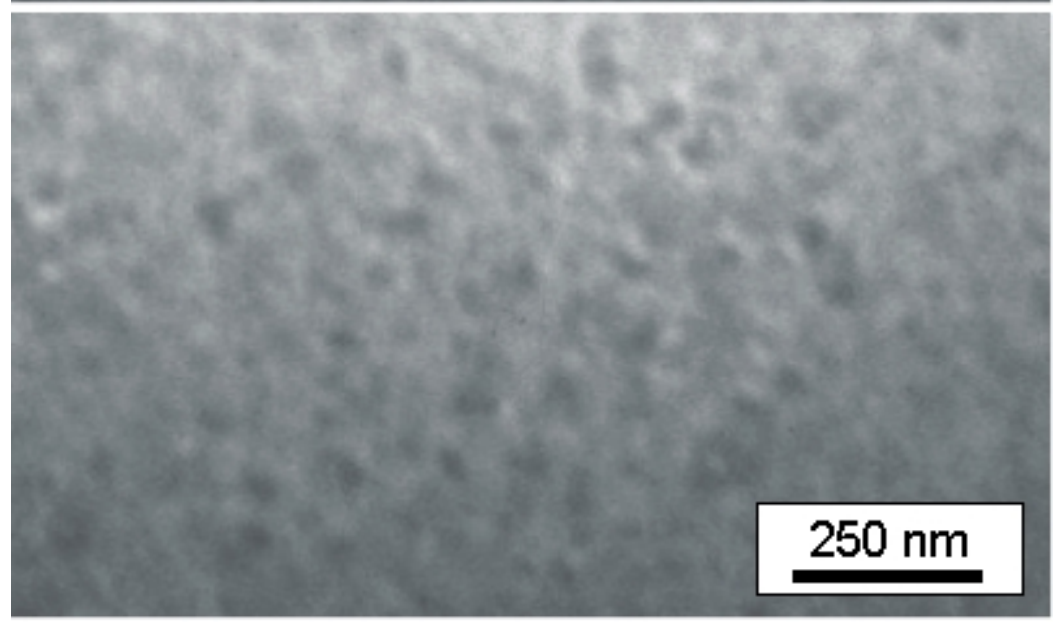

Fe/Ag:

$10 \mathrm{x} 2 \mathrm{~nm} / 10 \mathrm{~nm}$

$1 \mathrm{~h}$ bei $500^{\circ} \mathrm{C}$

Abbildung 4.6: Röntgenmikroskopische Abbildungen von desintegrierten Niob/Kupfer-, Eisen/Gold- und Eisen/Silber-Vielfachschichten. Das Niob/Kupfer-Schichtpaket enthält 3 Doppellagen, die Eisen/Gold- und Eisen/Silber-Schichtpakete 10 Doppellagen. 
nach wie vor keine Kontraste. Abbildung 4.6 gibt charakteristische Ausschnitte aus den so gewonnen Bildern wieder. Auffällig ist der deutliche Unterschied in der Skalierung der Kontraste. Während man bei der Niob/Kupfer-Schicht breite dunkle Flächen (niobreiche Stellen) erkennt, die durch helle Pfade (niobarme Stellen) voneinander getrennt werden, sind bei den Eisen/Gold- und Eisen/Silber-Schichten sehr viel kleinere helle, ovale Kontraste (eisenreiche Stellen) in einer dunklen Matrix zu sehen. Eine Auswertung der Intensitäten im System Niob/Kupfer offenbart, dass sich die großen Partikel aus mehreren Partikeln in verschiedenen Ebenen zusammensetzen, die in der Projektion als eine Einheit erscheinen. Berechnungen haben eine laterale Ausdehnung der einzelnen Partikel von etwa 80 bis100 nm ergeben. In Richtung parallel zum Strahl konnte die Dicke der Partikel durch die Stärke der Absorption zu Werten zwischen 20 und $40 \mathrm{~nm}$ bestimmt werden. Diese stimmen mit den Werten aus der Röntgenbeugung gut überein. Die Partikelanordnung ist annähernd statistisch, daher ist mit zunehmender Doppellagenzahl kein Kontrast mehr erkennbar (vgl. Kapitel 2.3). In den Systemen Eisen/Gold und Eisen/Silber ist das anders. Hier sind auch bei 10 Doppellagen punktförmige Kontraste zu erkennen. Im Falle des Eisen/Gold haben die Spots eine mittlere Abmessung von $11 \mathrm{~nm}$ entlang der kürzeren und $15 \mathrm{~nm}$ entlang der längeren Achse. Die Eisen/Silber-Proben erzeugen Kontraste mit einer Abmessung von 40 bis $60 \mathrm{~nm}$ lateraler Ausdehnung. Die Partikel müssen in Säulen senkrecht zur Schicht angeordnet sein, die leicht aus der Senkrechten verkippt sind, wodurch die Spots oval erscheinen. Der mittlere Abstand der Spots liegt im Eisen/Gold relativ eng verteilt bei etwa $10 \mathrm{~nm}$ und im Eisen/Silber bei etwa 20-25 nm. Aufgrund der Helligkeit der einzelnen Punkte kann davon ausgegangen werden, dass nicht immer gleich viele Partikel in jeder Säule liegen. Nur Punkte, die sehr hell erscheinen, sind Projektionen sehr vieler Eisenpartikel.

Als Ergebnis der röntgenmikroskopischen Untersuchungen kann festgehalten werden, dass im System Niob/Kupfer eine weitgehend statistische Bildung von Partikeln in den Lagen stattfindet, wogegen sich in den Systemen Eisen/Gold und Eisen/Silber eine senkrecht zur Schicht geordnete Struktur einstellt. In der Ebene haben die Partikelketten zwar eine enge Abstandsverteilung, scheinen aber nicht regelmäßig geordnet zu sein. 


\subsubsection{Transmissionselektronenmikroskopische Untersuchungen}

Um die Lage der einzelnen Partikel, sowie deren Größenverteilung untersuchen zu können, wurden an den ausgelagerten Proben TEM-Untersuchungen durchgeführt. Es zeigt sich dabei, dass die Partikelbildung im System Niob/Kupfer innerhalb der Lagen tatsächlich im Wesentlichen statistisch abläuft. An einigen Stellen finden sich Bereiche, an denen zwei oder drei Partikel direkt übereinander gebildet wurden. Solche Stellen sind dafür verantwortlich, dass im Röntgenmikroskop Kontraste zu erkennen waren. Darüber hinaus lässt sich anhand der Partikelpositionen noch die ursprüngliche Lagenstruktur nachvollziehen. Das bedeutet, dass die Partikel tatsächlich in den Ebenen gebildet wurden. Anhand der Menge Niob, die in den Partikeln der einzelnen Ebenen liegt, kann außerdem abgeschätzt werden, dass sich die jeweiligen Partikel aus dem Material der entsprechenden Ebenen gebildet haben. Eine Diffusion aus anderen Ebenen scheint nicht in nennenswerter Menge stattgefunden $\mathrm{zu}$ haben. Eine sehr wichtige Besonderheit an den desintegrierten Niob/Kupfer-Schichtpaketen ist die Stabilität der ersten zwei Doppellagen. Diese direkt auf das glatte Substrat aufgewachsenen Lagen sind die einzigen Lagen im gesamten Schichtpaket, die nicht von der Desintegration betroffen waren. Einziger Unterschied dieser ersten gegenüber den anderen Lagen ist, dass sie aufgrund der Nähe zum Substrat extrem glatt aufgewachsen sind. In den TEM-Bildern der unbehandelten Proben lässt sich erkennen, dass sich danach rasch eine Rauigkeit in den Schichten aufgebaut hat. Näheres zu diesen Untersuchungen ist in [Troche, 1996] zu

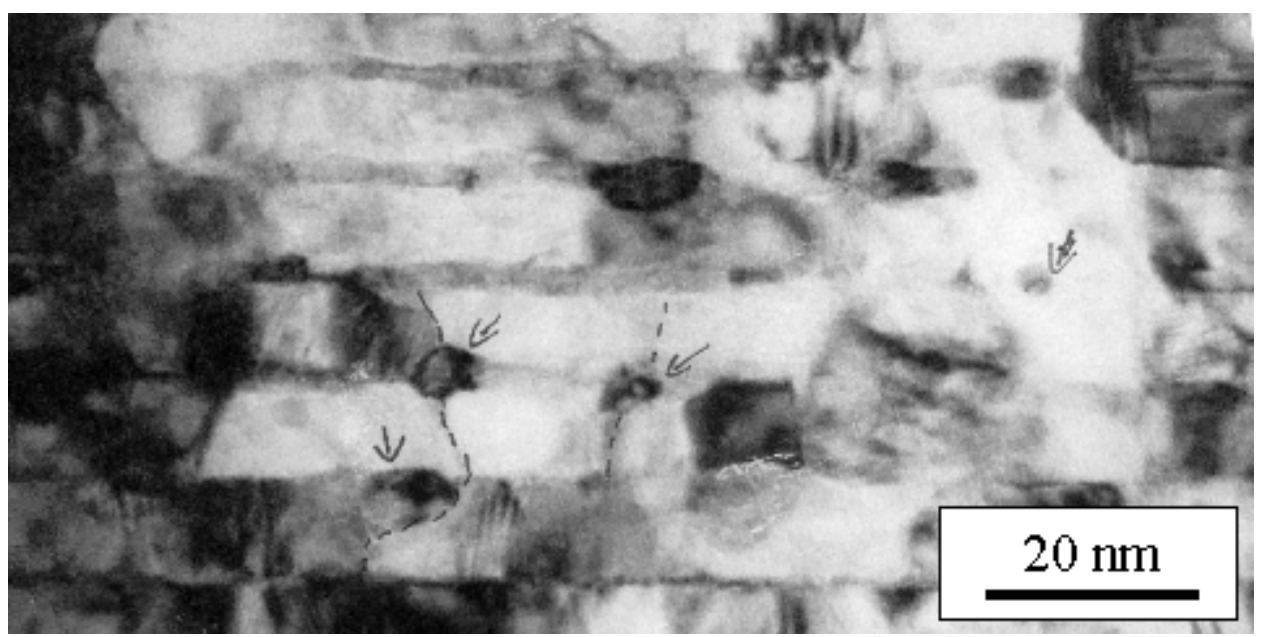

Abbildung 4.7: Anfangsstadium der Desintegration einer Niob/KupferVielfachschicht $(2 \mathrm{~nm} / 10 \mathrm{~nm})$. Viele der ersten Partikel bilden sich in oder an Korngrenzen der Kupferschicht. 
finden. Ein weiteres Detail der Desintegration findet sich in Abbildung 4.7, das den Beginn der Desintegration einer Niob/Kupfer-(3 nm/15 nm)-Vielfachschicht zeigt. Die Probe wurde 24 Stunden bei $550^{\circ} \mathrm{C}$ ausgelagert. Aufgrund der Untersuchungen zur Zerfallstemperatur in Abhängigkeit von der Lagendicke, war für Proben mit $3 \mathrm{~nm}$ Nioblagendicke keine Desintegration bei $550^{\circ} \mathrm{C}$ zu erwarten. Es zeigt sich, dass sich das Schichtpaket tatsächlich noch in einem sehr frühen Stadium der Desintegration befindet. Nur an wenigen Stellen haben sich nach der langen Auslagerungszeit kleine Partikel gebildet. Hier ist zu erkennen, dass ein auffällig großer Anteil dieser Partikel in oder an Korngrenzen der Kupfermatrix gebildet wurden. Wenn diese Kupferkorngrenzen für die Bildung von Niobpartikeln bevorzugte Orte darstellen, würde das erklären, warum die Niobpartikel zum Teil geordnet übereinander aufwachsen, im wesentlichen jedoch statistisch angeordnet sind. Die Kupferkörner haben nur eine kleine laterale Ausdehnung und nur bei größeren Körnern wird deren Orientierung über zwei bis drei Lagen weitergegeben. Ordnung würde also immer dort auftreten, wo solche großen Kupferkörner in der Probe gebildet wurden. An dieser Probe zeigt sich auch, dass die Desintegrationstemperatur kein

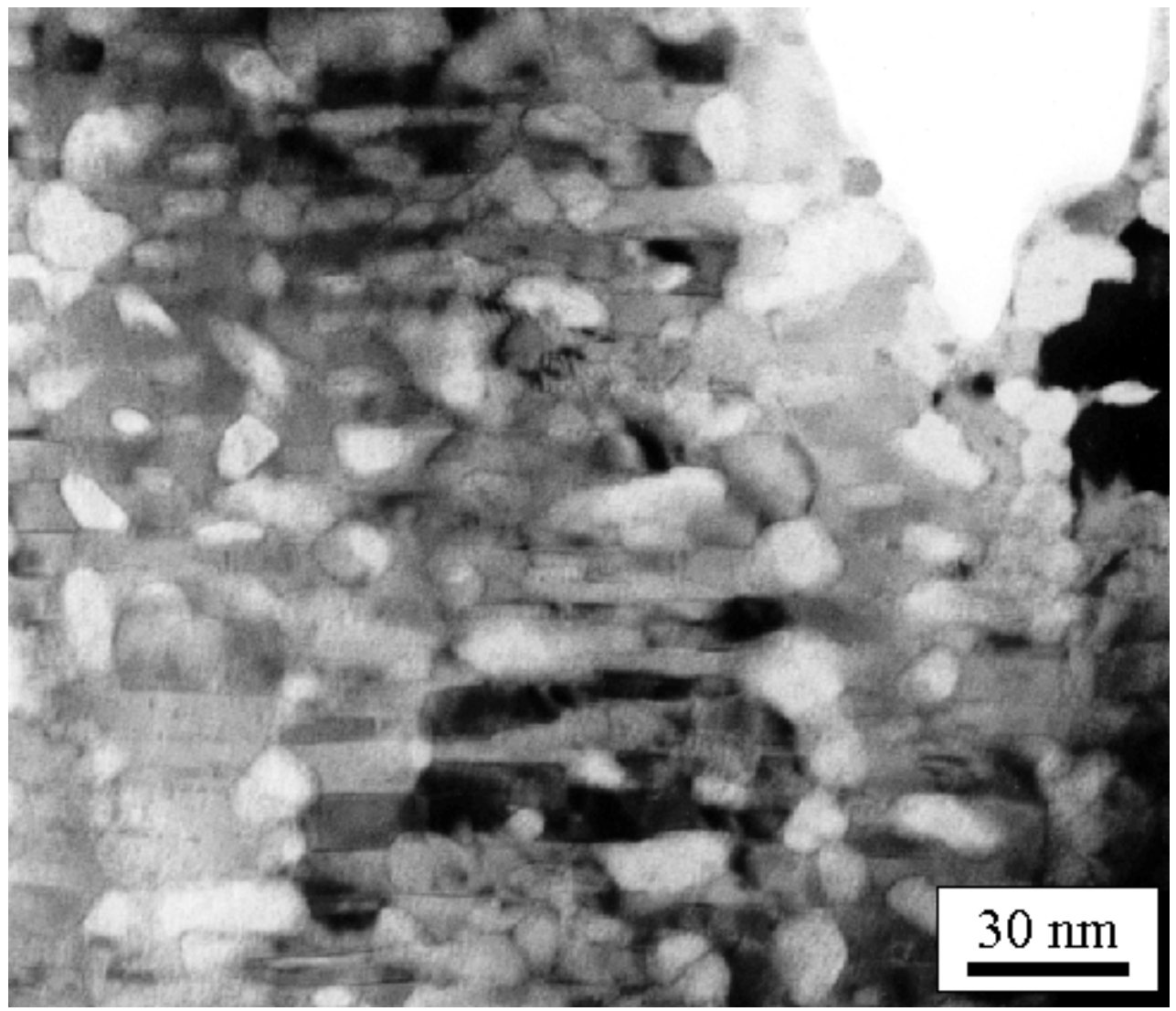

Abbildung 4.8: Desintegrierte Eisen/Gold-(2 $\mathrm{nm} / 4 \mathrm{~nm})$-Vielfachschicht. Auslagerung 30 Minuten bei $330^{\circ} \mathrm{C}$. 
absolute Schranke für die Desintegration darstellt, sondern nur einen Wert angibt, unterhalb welchem die Desintegration sehr langsam abläuft.

Wie aus den röntgenmikroskopischen Untersuchungen $\mathrm{zu}$ erwarten war, ist in den Systemen Eisen/Gold und Eisen/Silber eine deutlich geordnetere Mikrostruktur zu erkennen. Für beide Systeme wurde neben den nor-

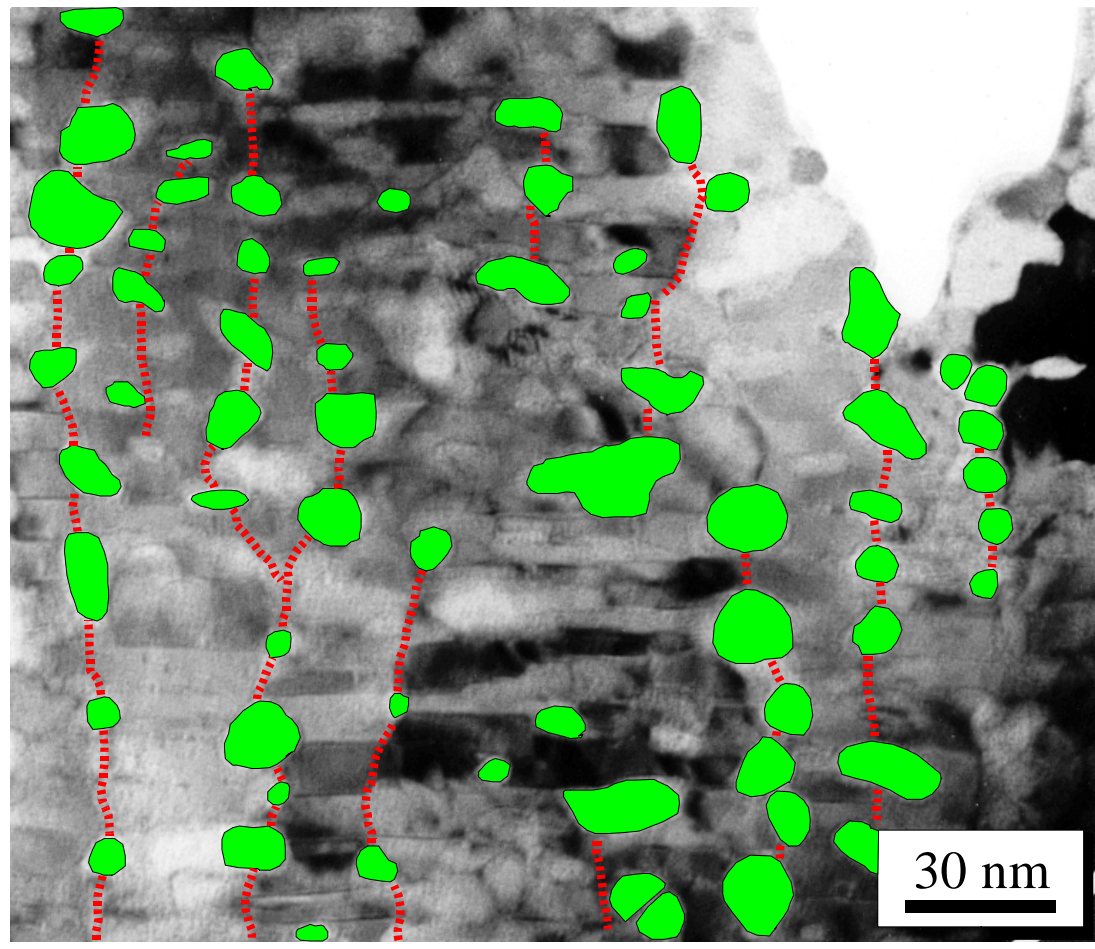

Abbildung 4.9: (wie Abbildung 4.8) Zur besseren Übersichtlichkeit sind die Eisenpartikel in hellgrün und die Goldkorngrenzen in rot gekennzeichnet.

malen Hellfeldbildern

auch Z-Kontrast-Aufnahmen gemacht, um die Eisenpartikel eindeutig von der Edelmetallmatrix unterscheiden zu können. Abbildung 4.8 zeigt ein Hellfeldbild einer 30 Minuten bei $330^{\circ} \mathrm{C}$ ausgelagerten Eisen/Gold- $(2 \mathrm{~nm} / 10 \mathrm{~nm})-$ Vielfachschicht. In Abbildung 4.9 ist zur besseren Übersicht über die Anordnung der Eisenpartikel das gleiche Bild mit hellgrün markierten Eisenpartikeln und rot markierten Goldkorngrenzen gezeigt. Die Eisenpartikel haben sich bevorzugt in den ehemaligen Makrokorngrenzen gebildet und zeigen entsprechend das im Röntgenmikroskop beobachtete Ordnungsverhalten senkrecht zur Schicht. Es ist auffällig, dass die grobe Mikrostruktur von der Desintegration nicht beeinflusst wurde. Das bedeutet, dass die Makrokörner noch klar zu erkennen sind und dass auch in den Goldlagen kein Kornwachstum stattgefunden hat. Verändert hat sich allerdings die Krümmung der Grenzflächen. Hier erkennt man, dass sich die Grenzflächen während der Desintegration geglättet haben und die gleich orientierten Goldlagen zusammengewachsen sind.

Qualitativ erhält man im System Eisen/Silber das gleiche Ergebnis wie im System Eisen/Gold. Untersucht wurde eine 60 Minuten bei $500^{\circ} \mathrm{C}$ ausgelagerte Schicht. In 


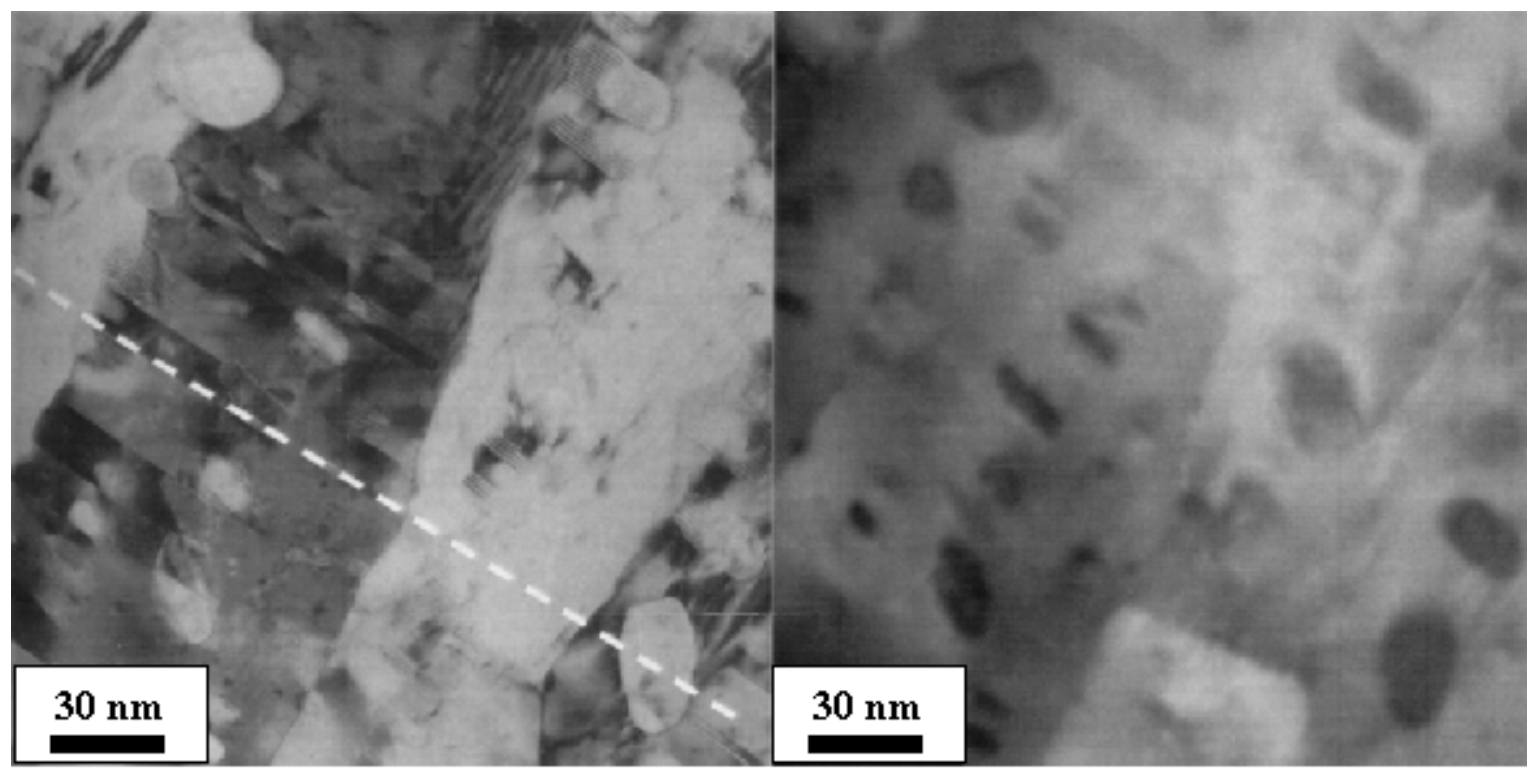

Abbildung 4.10: TEM-Bilder einer 60 Minuten bei $500^{\circ} \mathrm{C}$ ausgelagerten Eisen/Silber $(2 \mathrm{~nm} / 10 \mathrm{~nm})$ Vielfachschicht. Links: Hellfeldbild. Rechts: Z-Kontrastbild [Herweg, 1999].

Abbildung 4.10 sind ein Hellfeldbild (links) und zum Vergleich ein Z-Kontrast-Bild derselben Probenstelle abgebildet. Auch hier haben sich die Eisenpartikel in den Korngrenzen der Makrokörner gebildet. Diese sind durch ihre eher runde Form gut zu erkennen. Daneben haben sich aber auch diskusförmige Partikel innerhalb der Makrokörner gebildet. Auffällig ist, dass deren Mittelachsen genau in den nahezu perfekt glatten Grenzflächen liegen. Auch in diesem System hat sich die grobe Mikrostruktur nicht verändert, die Makrokörner sind vollständig erhalten und die Silberlagen sind nach Verschwinden des Eisens zusammengewachsen.

Es ist interessant, einen Blick auf die Partikelgrößen, besonders aber auf die Streuung der Größenverteilung und die Exzentrizität der Partikel zu werfen. Die mittlere Partikelgröße der Niobpartikel lässt sich aus den TEM-Bildern zu $23 \mathrm{~nm}$ mit einer Standardabweichung von $40 \%$ berechnen. Zur Bestimmung wurden die Breite und Höhe der Nanopartikel gemessen. Um eine gute Vergleichbarkeit der Daten zu gewährleisten, wurde aus Höhe und Breite das Volumen eines Rotationsellipsoids berechnet und daraus der Durchmesser einer Kugel gleichen Volumens abgeleitet. Um die Symmetrie der Partikel darzustellen, wurde die Exzentrizität, die der Quotient aus Breite und Höhe der Partikel ist, berechnet ${ }^{3}$. Im Fall des Niob/Kupfer beträgt sie genau 1, allerdings mit einem großen Fehler von 40\%. Das

3 Für die Statistik der Partikelgrößen wurden für jedes System 80 Partikel ausgewertet. 
lässt darauf schließen, dass die Partikel annähernd kugelförmig sind, was auch für viele Partikel zutrifft. Es ist allerdings zu beachten, dass hier einige Partikel eine Exzentrizität größer als eins und einige eine kleiner als eins aufweisen, was bei den anderen Systemen nicht beobachtet wird. Im System Eisen/Gold betrug die Partikelgröße 9,6 nm und hatte eine nur halb so große Streuung von $20 \%$ wie die im System Niob/Kupfer. Die Exzentrizität ist allerdings deutlich größer, sie beträgt hier 1,49, was bedeutet, dass die Partikel im Mittel 50\% breiter als hoch sind. Für die desintegrierte Eisen/Silber-Schicht ergab sich eine mittlere Partikelgröße von 12,8 nm mit einer Abweichung von 23\%. Die Exzentrizität lag hier ähnlich wie die des Eisen/Gold bei 1,47. Da es in diesem System ganz offensichtlich zwei Typen von Partikeln gibt, nämlich die innerhalb der Makrokörner und die innerhalb der Makrokorngrenzen, wurde zusätzlich eine Statistik für die jeweiligen Partikel angefertigt. Dabei ergab sich, dass die Partikel in den Korngrenzen größer sind als der Mittelwert, sie haben einen mittleren Durchmesser von 14,2 nm und eine Streuung von 25\%. Die Exzentrizität ist mit 1,14 deutlich kleiner als der Mittelwert und auch deutlich kleiner als der Wert im System Eisen/Gold. Für die diskusförmigen Partikel macht die Bestimmung eines Durchmessers eigentlich keinen Sinn. Es ist aber interessant, dass die Streuung der Größe nur $11 \%$ beträgt, was ungefähr ein Viertel des Wertes im Niob/Kupfer ist. Die Exzentrizität ist natürlich groß, sie beträgt 2,07. Das heißt, die Partikel sind im Mittel mehr als doppelt so breit wie hoch. Zur besseren Übersicht sind die Werte in Tabelle 4.1 nochmals zusammengefasst.

Bei der Betrachtung der Eisen/Edelmetall-Schichten ist auffällig, dass die Partikel zum großen Teil in Ketten vorliegen, deren Positionen durch die Makrokorngrenzen bestimmt sind. Sie haben Längen von bis zu 20 Doppellagen, in denen sie den Ma-

\begin{tabular}{|l|c|c|c|c|c|}
\hline & Fe/Ag & Fe/Ag rund & Fe/Ag oval & Fe/Au & Nb/Cu \\
\hline Durchmesser & $12,8 \mathrm{~nm}$ & $14,2 \mathrm{~nm}$ & $10,2 \mathrm{~nm}$ & $9,6 \mathrm{~nm}$ & $22,2 \mathrm{~nm}$ \\
\hline Abweichung & $23 \%$ & $25 \%$ & $11 \%$ & $20 \%$ & $40 \%$ \\
\hline Exzentrizität & 1,47 & 1,14 & 2,07 & 1,49 & 1,02 \\
\hline Abweichung & 0,46 & 0,18 & 0,43 & 0,41 & 0,37 \\
\hline
\end{tabular}

Tabelle 4.1: Exzentrizitäten und Partikeldurchmesser in den untersuchten Systemen nach der Auslagerung. 
krokorngrenzen folgen. Dieses Ergebnis erklärt, warum die röntgenmikroskopischen Bilder so gleichmäßig angeordnete gleich helle Punkte zeigen. Ihr Abstand wird durch die Breite der Makrokörner gegeben, die auf den TEM-Bildern eine geringe Streuung um einen Mittelwert aufweisen. In diesem Zusammenhang fällt auf, dass die Partikelketten im Röntgenmikroskop recht gleichmäßig verteilt scheinen, wogegen es im TEM scheint, als ob nur einige Makrokorngrenzen Nanopartikel enthielten. In solchen partikelfreien Bereichen stimmt auch die Menge Eisen, die vorher zwischen den Goldlagen vorhanden war nicht mit dem Eisengehalt in den umgebenen Nanopartikeln überein. Der Grund dafür liegt darin, dass bei einer querschnittpräparierten Probe nur ein Schnitt durch die Probe zu sehen ist. Auf Durchstrahlungsbildern wie in Abbildung 4.5 (Seite 41) gezeigt, wird ersichtlich, dass die Makrokörner facettierte Säulen sind. Da die Makrokorngrenzen offensichtlich bevorzugte Orte zur Bildung von Nanopartikeln sind, kann angenommen werden, dass die Punkte, an denen drei Makrokörner aufeinander treffen, für die Bildung der Nanopartikel besonders günstig sind. In diesem Fall würde man Abbildungen wie die hier vorgestellten erhalten, bei denen der Schnitt durch die Probe nur einige der Partikelketten trifft, während andere Tripelpunkte der Makrokörner vor oder hinter der Schnittebene liegen (Abbildung 4.11).

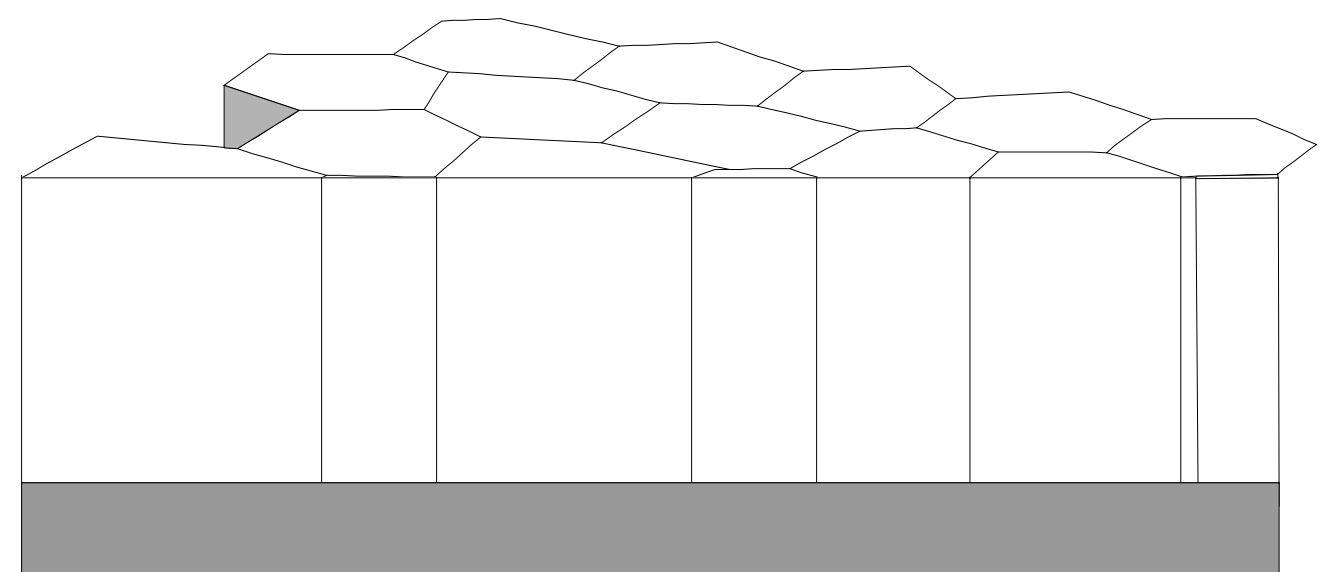

Abbildung 4.11: Skizze eines Schnittes durch die Makrokörner, wie er bei der Querschnittspräparation auftreten würde. 


\subsection{Desintegration in den Systemen Kobalt/Kupfer und Kobalt/Kohlenstoff}

Neben den zuvor vorgestellten Ergebnissen zur Desintegration von Schichtsystemen mit großen Gitterfehlpassungen soll anhand der Vergleichsysteme Kobalt/Kupfer und Kobalt/Kohlenstoff festgestellt werden, wie sich Vielfachschichten bei einer Auslagerung verändern, die entweder nur kleine oder gar keine Fehlpassung an den Grenzflächen aufweisen.

\section{Kobalt/Kupfer}

Eine Heizmessung an einer Kobalt/Kupfer-( $2 \mathrm{~nm} / 10 \mathrm{~nm})$-Vielfachschicht bei verschiedenen Temperaturen von Raumtemperatur in $100^{\circ} \mathrm{C}$-Schritten auf $500^{\circ} \mathrm{C}$ und anschließender Abkühlung (Abbildung 4.12) offenbart den grundlegenden Unterschied zwischen dieser Vielfachschicht und den zuvor besprochenen. Während zunächst keine Überstrukturen im Diffraktogramm zu erkennen sind, entwickeln sich diese bei Temperaturen wenig über $300^{\circ} \mathrm{C}$ und bleiben danach auch bei höheren Temperaturen bestehen. Auch nach einer Auslagerung bei $600^{\circ} \mathrm{C}$ hatte die Probe keine Mikrostrukturveränderung durchlaufen. Erst oberhalb von $700^{\circ} \mathrm{C}$ verschwin-

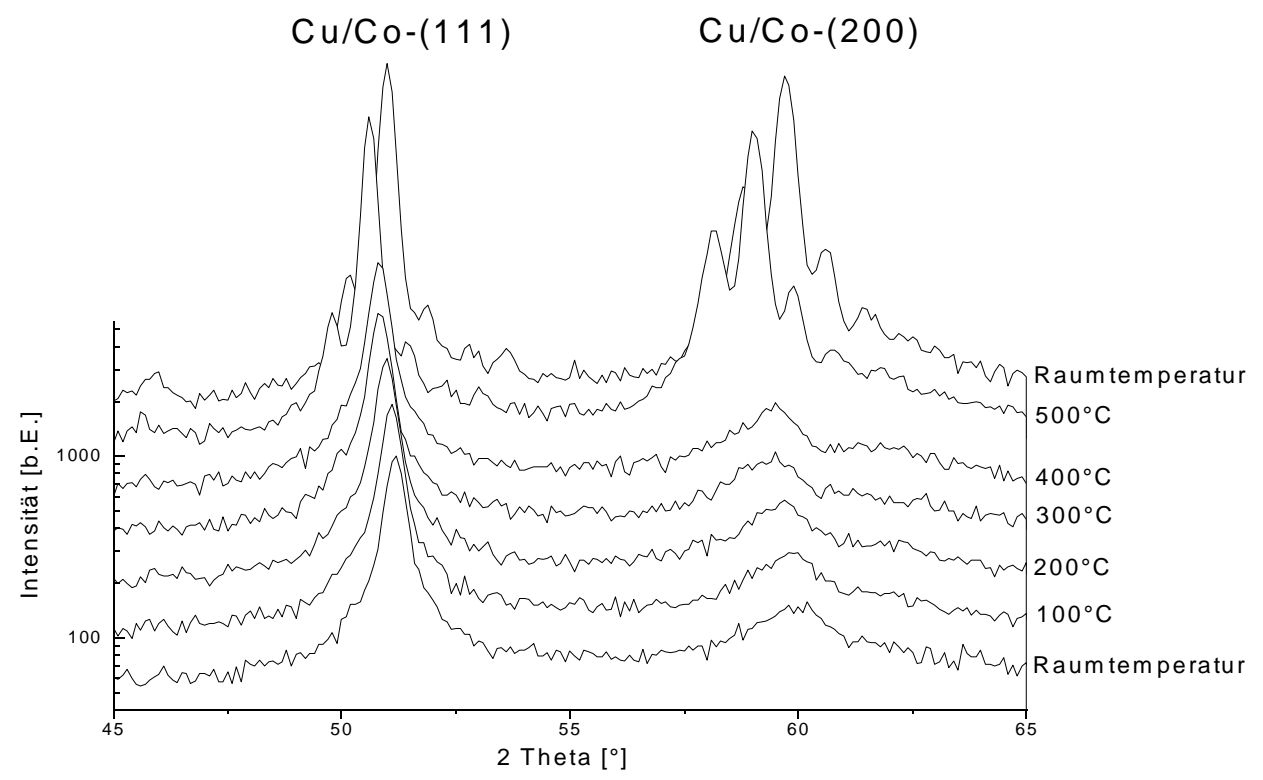

Abbildung 4.12: Kobalt/Kupfer-(2 $\mathrm{nm} / 10 \mathrm{~nm})$-Vielfachschicht während einer stufenweise ansteigenden Auslagerung: $\mathrm{RT}, 100^{\circ} \mathrm{C}, 200^{\circ} \mathrm{C}, 300^{\circ} \mathrm{C}, 400^{\circ} \mathrm{C}, 500^{\circ} \mathrm{C}$. Beim Übergang von $300^{\circ} \mathrm{C}$ auf $400^{\circ} \mathrm{C}$ wird eine klare Überstruktur der Probe sichtbar. 
det die Überstruktur und die reinen Kupfer- und kubisch-flächenzentrierten KobaltReflexe werden sichtbar. Im Gegensatz zu den bisher betrachteten Systemen wird im System Kobalt/Kupfer eine ursprünglich vorhandene Grenzflächenrauigkeit bei der Auslagerung verringert. Sämtliche Reflexe, die an Kobalt/Kupfer Proben verschiedener Lagendickenverhältnissen gemessen wurden, zeigen, dass das Kobalt, dessen Lagendicke $2 \mathrm{~nm}$ nie überstieg, vom Kupfer in die kubisch-flächenzentrierte Struktur gezwungen wurde. Hexagonale Anteile an den Kobaltreflexen konnten weder in den wie-hergestellten Proben, noch aus Anpassungen an die Überstrukturen gefunden werden.

Des Weiteren entnimmt man der Abbildung 4.12, dass sich mit dem Auftreten der Überstruktur die Intensität vom gemeinsamen (111)-Reflex zum gemeinsamen (200)-Reflex hin verschiebt. Besonders deutlich ist dieser Effekt in Schichtpaketen mit kleinen Periodenlängen (z.B. Kobalt/Kupfer- (1,5 nm/4 nm)-Schichten). Diese Proben weisen im wie-hergestellten Zustand eine fast reine (111)-Drahttextur auf, die sich während der Auslagerung in eine fast reine (200)-Drahttextur verändert. Während dieser Umwandlung findet eine Glättung der Grenzflächen statt, die zur Ausbildung von Überstrukturen führt. Es wurden ähnliche Übergänge auch an sehr dünnen Kobaltschichten auf Saphir beobachtet [Shi et al., 1998]. Dabei wurde bei den unbehandelten Schichten ebenfalls hauptsächlich eine flächenzentrierte (111)Orientierung festgestellt, die dann bei einer kritischen Temperatur in eine (100)Orientierung über ging. In diesem Fall verlief die Umwandlung sogar reversibel, so dass bei unterschreiten der Übergangstemperatur wieder die (111)-Phase eingestellt wurde. Im hier vorgestellten Fall ist der Übergang zwischen den Orientierungen nicht reversibel, sondern läuft nur bei Überschreiten der Übergangstemperatur in eine Richtung ab. Diese Übergangstemperatur liegt aber bei allen hier untersuchten Schichten unabhängig von der Zusammensetzung der Lagenpakete bei etwa $330^{\circ} \mathrm{C}$. Dabei wurden Vielfachschichten mit $1 \mathrm{~nm}$ bis $3 \mathrm{~nm}$ Kobalt und $3 \mathrm{~nm}$ und $10 \mathrm{~nm}$ Kupfer untersucht. Die schwache Intensität und die große Halbwertsbreite der Reflexe der unbehandelten Schichten lassen auf ein nanokristallines Gefüge schließen. Es liegen somit große treibende Kräfte für die Rekristallisation vor. Setzt man weiter voraus, dass die Grenzflächenenergie zwischen (100) orientierten Kobalt/Kupfer-Grenzflächen kleiner als für (111) orientierte Grenzflächen ist, könnten (100) orientierte Kobalt- und Kupferkörner mit einer gemeinsamen Grenzfläche als Re- 
kristallisationskeime dienen. Diese Annahme ist nach dem Beckerschen Modell [Becker, 1938] (siehe auch Kapitel 6.1) sinnvoll, da es die Grenzflächenenergie über die Zahl der Bindungen abschätzt. Folglich ist die Energie der (100)-Grenzfläche um einen Faktor 1,2 kleiner als die einer (111)-Grenzfläche. Das rekristallisierte Gefüge weist eine (200)-Textur und minimierte, sprich glatte Grenzflächen auf. Dieser Prozess erscheint sinnvoll, da im System Kobalt/Kupfer keine Mischkristallbildung möglich ist. Diese Vorstellung erklärt auch plausibel, dass für dickere Kupferlagen die Umwandlung nicht vollständig ist. Mit zunehmender Vergrößerung der Körner im Kupfer verringern sich die treibenden Kräfte der Rekristallisation bei relativ tiefer Temperatur von $330^{\circ} \mathrm{C}$ und die Grenzflächenbeiträge verlieren im Vergleich zu anderen volumenabhängigen Beiträgen an Gewicht.

TEM-Aufnahmen von Kobalt/Kupfer-Vielfachschichten sind schwierig herzustellen, da die Metalle aufgrund ihrer ähnlichen Massen und Streufaktoren nur einen sehr schwachen Kontrast erzeugen. Verschiebt man die Probe leicht aus dem Fokus des Elektronenstrahls, erhält man einen leichten Phasenkontrast, der es ermöglicht bei schwacher Auflösung eine Abbildung zu erzeugen. In Abbildung 4.13 ist eine Vielfachschicht nach einer 30minütigen Auslagerung bei $350^{\circ} \mathrm{C}$ gezeigt. Auch hier sind Ansätze eines kolumnaren Wachstums zu erkennen, allerdings sind die Grenzflächen hier nicht gekrümmt. Das zeigt die geringe Spannung an der Grenzfläche. Deutlich zu erkennen ist die sehr periodische Struktur der Probe, die keinerlei Bil-
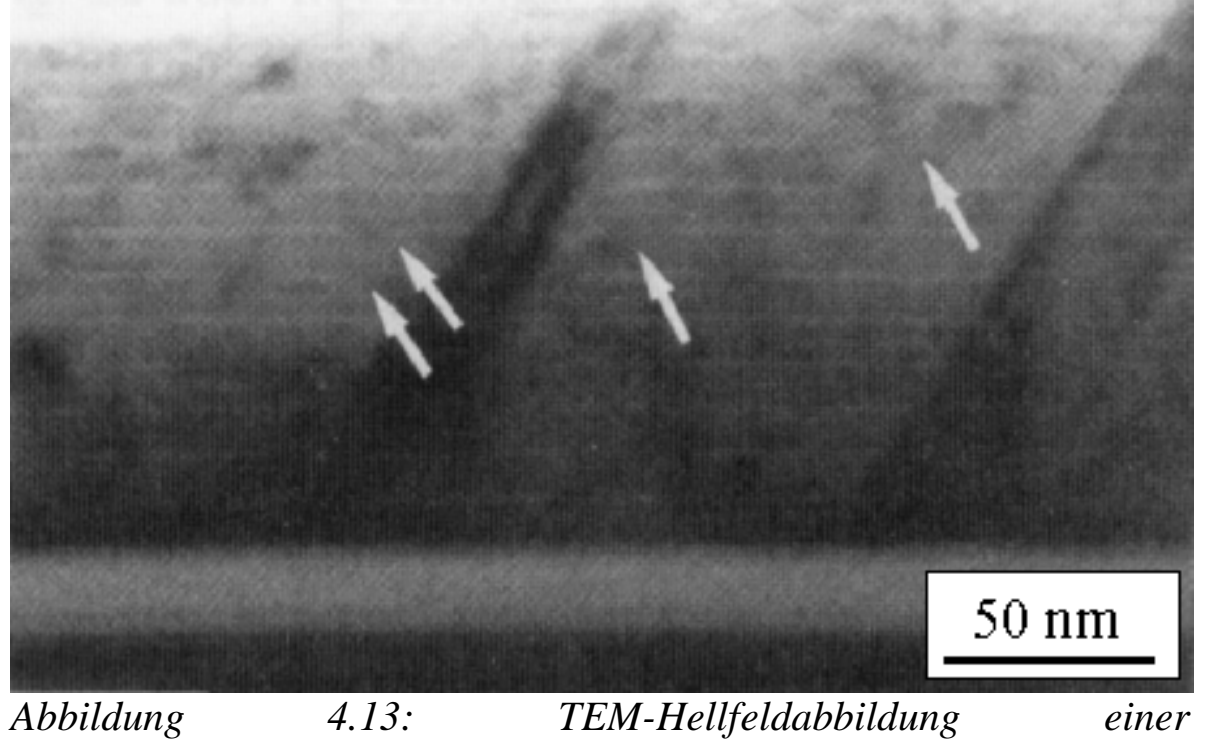
Kobalt/Kupfer-(1,5 nm/4 nm)-Vielfachschicht nach 30 minütiger Auslagerung bei $350^{\circ} \mathrm{C}$ [Rätzke et al., 1999]. (Pfeile ohne Bedeutung) 
dung von Nanopartikeln zeigt. Das TEM-Bild bestätigt damit die Annahmen, die aus den Diffraktogrammen hergeleitet wurden.

\section{Kobalt/Kohlenstoff}

Die Kobalt/Kohlenstoff Vielfachschichten waren die glattesten und stabilsten Schichten, die im Rahmen dieser Arbeit untersucht wurden. Während der Auslagerung zeigten die Kleinwinkelreflexe bis hinauf $\mathrm{zu} 750^{\circ} \mathrm{C}$ keine Veränderung ihrer Intensitäten. Da die Kleinwinkelreflexe besonders empfindlich auf Veränderungen der Grenzfläche reagieren, kann daraus sicher geschlossen werden, dass sich die Rauigkeit der Schichten nicht verändert hat und auch keine Kristallisation des amorphen Kohlenstoffs stattgefunden hat. Konno et al. [Konno et al., 1994] haben gezeigt, dass Kobalt/Kohlenstoff- $(20 \mathrm{~nm} / 40 \mathrm{~nm})$-Schichten bei $500^{\circ} \mathrm{C}$ desintegrieren. In ihren Untersuchungen stellten sie fest, dass die Zerstörung der Lagenstruktur dabei direkt mit der Kristallisation des amorphen Kohlenstoff korreliert ist. In Abbildung 4.14 ist die Abhängigkeit der Kristallisationstemperatur des Kohlenstoff von der Lagendicke des Kobalt dargestellt. Diese steigt mit abnehmender Kobaltlagendicke an. Da in den hier vorgestellten Experimenten nur $2 \mathrm{~nm}$ dicke Kobaltlagen hergestellt wurden, erwartet man nach diesen Messungen eine Kristallisationstemperatur deutlich oberhalb von $600^{\circ} \mathrm{C}$. Dies kann erklären, warum die Kohlenstoffschichten bis $750^{\circ} \mathrm{C}$ amorph blieben und somit auch keine Desintegration der

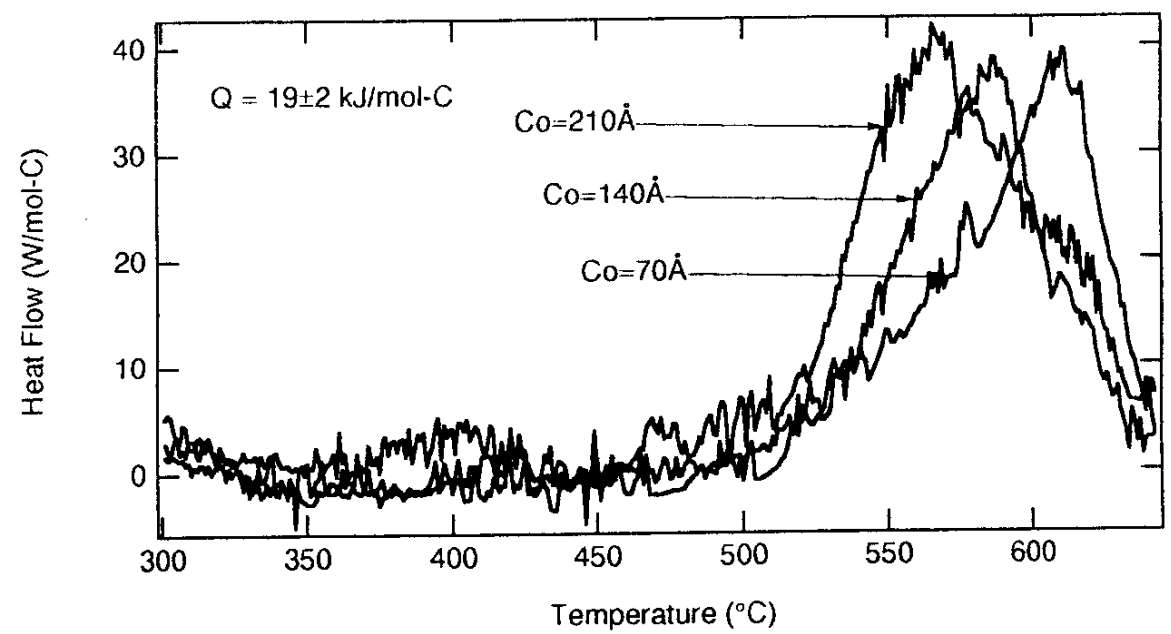

Abbildung 4.14: Kalorimetrische Messungen an Kohlenstoff/Kobalt Dreifachschichten. Mit abnehmender Kobaltlagendicke verschiebt sich die Kristallisation des amorphen Kohlenstoff zu höheren Temperaturen [Konno et al., 1994]. 
Schichtpakete beobachtet wurde.

Diese Stabilität wird jedoch nicht in allen Metall/Kohlenstoff-Vielfachschichten gefunden. Bobeth et al. [Bobeth et al., 1997] beobachteten an Nickel/KohlenstoffSchichten eine Desintegration bei ungefähr $400^{\circ} \mathrm{C}$. Diese wurde verursacht durch die unterschiedlichen Grenzflächenspannungen von Nickel/Nickel- und Nickel/Kohlenstoff-Grenzflächen. Dadurch kerbten sich die Grenzflächen an den Nickelkorngrenzen ein und bildeten Löcher in der Nickelschicht. Dieser Prozess wurde durch die leichte Beweglichkeit der Kohlenstoffatome begünstigt.

\subsection{Vergleich der Mikrostruktur nach der Auslagerung}

Vergleicht man die Desintegration der verschiedenen Schichtsysteme, so werden sowohl bei der Mikrostruktur nach der Auslagerung, wie auch bei der Desintegrationstemperatur deutliche Unterschiede sichtbar. Die Desintegrationstemperatur liegt bei dem stark verspannten System Eisen/Gold mit seinen regelmäßig gekrümmten Grenzflächen mit $\mathrm{T}_{\text {Zerf }}>325^{\circ} \mathrm{C}$ für Vielfachschichten mit $2 \mathrm{~nm}$ Eisen und $10 \mathrm{~nm}$ Gold am niedrigsten. Beim System Eisen/Silber liegt sie, anders als aufgrund der strukturellen Ähnlichkeit der Systeme vielleicht erwartet, mit $\mathrm{T}_{\text {Zerf }}>450^{\circ} \mathrm{C}$ deutlich höher. Niob/Kupfer Vielfachschichten mit der gleichen Zusammensetzung bleiben bis $\mathrm{T}_{\text {Zerf }}>500^{\circ} \mathrm{C}$ stabil. Kupfer/Kobalt bleibt selbst bei sehr dünnen Lagendicken von weniger als einem Nanometer bis über $700^{\circ} \mathrm{C}$ stabil. Allein das System Kobalt/Kohlenstoff zerfiel selbst bei Wärmebehandlungen bis $750^{\circ} \mathrm{C}$ nicht. Aus den vorgestellten Ergebnissen von den Systemen Niob/Kupfer und Eisen/Gold wurde aber auch ersichtlich, dass die Desintegrationstemperatur stark von den Lagendicken der interkalierten Lagen abhängig ist. In beiden Systemen wird unterhalb einer kritischen Lagendicke eine reziproke Abhängigkeit der Desintegrationstemperatur von der Lagendicke beobachtet. Der Wert der kritischen Lagendicke ist in den verschiedenen Systemen jedoch unterschiedlich. Für das System Eisen/Silber konnte eine solche Kurve experimentell nicht realisiert werden, da die Desintegrationstemperatur aufgrund der fehlenden Überstrukturen nicht im Rahmen der erforderlichen Genauigkeit zu bestimmen war. Der Grund für die bei den ersten beiden Systemen gezeigte Abhängigkeit wird im nächsten Abschnitt diskutiert werden.

Auch die Mikrostruktur der vorgestellten Systeme unterscheidet sich nach der 
Auslagerung stark voneinander. Das System Niob/Kupfer ist das am wenigsten geordnete System, was sich in der starken Streuung der Partikelgrößen von $40 \%$ widerspiegelt. Stark unterschiedliche Partikelgrößen sind dabei direkt mit einer ungleichmäßigen Verteilung korreliert, da die Partikel im Wesentlichen aus den einzelnen Lagen gebildet werden. Anders als in den anderen Systemen sind die Partikel annähernd kugelförmig. Die Größenverteilung im System Eisen Gold streut nur um 20\%, die Partikel sind im Mittel 1,5 mal so breit wie hoch. Wiederum anders ist die Verteilung der Partikel im System Eisen/Silber, wo sich in den Makrokorngrenzen kugelförmige und innerhalb der Makrokörner diskusförmige Partikel gebildet haben. Die Streuung ersterer entspricht der im System Eisen/Gold, wogegen die diskusförmigen Partikel eine sehr enge Größenverteilung mit nur 11\% Abweichung zeigen. Aus der gleichmäßigen Größenverteilung der letzten Systeme lässt sich auch eine regelmäßigere Mikrostruktur ableiten, die sich in den Systemen auch dadurch zeigt, dass die Partikel in den Korngrenzen teilweise Ketten bilden, deren Abstand durch die Breite der Makrokörner vorgegeben sind.

Eine weitere Beobachtung, die an allen desintegrierten Vielfachschichten gemacht wurde, ist die unveränderte Textur der Schichten. In den verschiedenen Darstellungen der Mikrostruktur der Proben war eine Neigung der Drahttextur in Richtung der Quellen je nach dem Anteil ihrer Lagendicken $\mathrm{zu}$ erkennen. Eine Desintegration, die durch eine Keimbildung und anschlieBendes Kornwachstum entsteht, würde diese Kippung der Textur aufheben oder zumindest verändern. Es bleibt jedoch in allen untersuchten Schichten bei der Kippung in die gleiche Richtung (Abbildung 4.15).

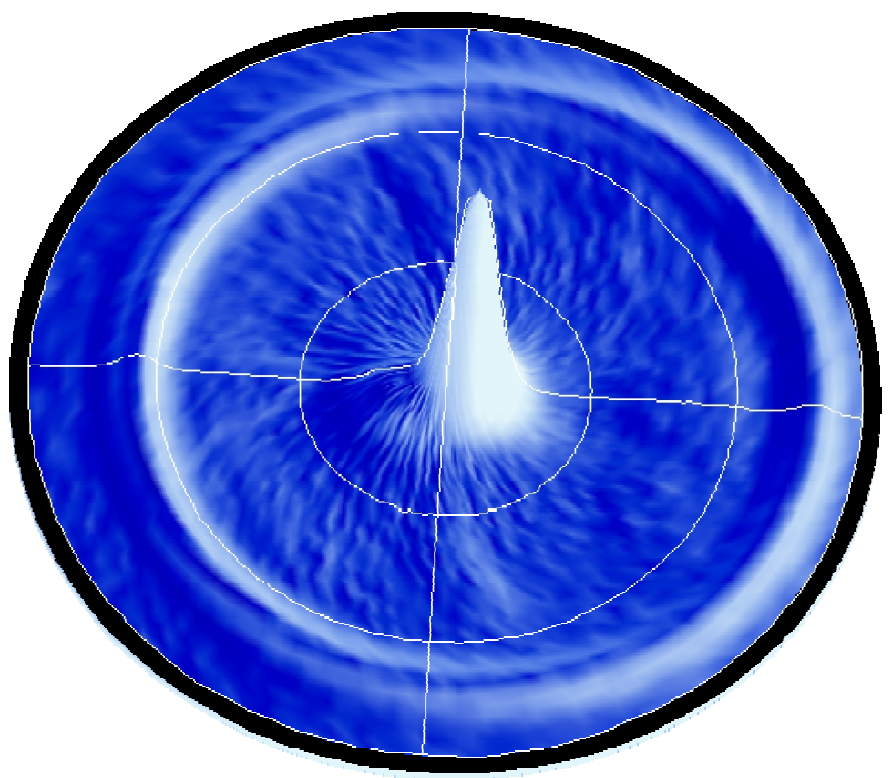

Abbildung 4.15: Drahttextur einer desintegrierten Eisen/Gold-Vielfachschicht. Hier gemessen der Gold-(111)-Reflex. Vgl. Textur der unbehandelten Vielfachschicht (Abbildung 3.8, Seite 28). 


\section{Treibende Kräfte einer Desintegration}

Die Desintegration von Vielfachschichten während einer thermischen Behandlung ist ein Phänomen, das in der Literatur noch nicht abschließend erklärt wurde, da sehr viele Parameter in diesem Prozess berücksichtigt werden müssen. Dominierenden Einfluss auf die Desintegration hat offensichtlich die Mikrostruktur der Schichten nach der Herstellung, die stark vom Herstellungsprozess und der Depositionstemperatur abhängt. Aber auch die Chemie der einzelnen Systeme, d.h. z.B. die Paarvertauschungsenergie oder die Löslichkeit, die aus den Phasendiagrammen ersichtlich wird, scheint einen starken Einfluss zu haben. Das wird durch den Unterschied der Desintegration zwischen den Systemen Eisen/Gold und Eisen/Silber offensichtlich.

Ein Ansatz, der für Modellsysteme theoretisch berechnet [Cline, 1971], [van Suchtelen, 1977] und für reale Systeme zur teilweisen Erklärung herangezogen wurde [Bormann, 1979], ist die lokale Variation der Grenzflächenrauigkeit. Der Krümmungsradius der Grenzfläche hat einen Einfluss auf das chemische Potenzial der Grenzfläche und kann so einen Diffusionsstrom parallel zur Grenzfläche antreiben. Ebenfalls hat die mechanische Grenzflächenspannung Einfluss auf das chemische Grenzflächenpotenzial. Eine Variation der Grenzflächenspannung kann ebenfalls einen Diffusionsstrom parallel zur Grenzfläche von Stellen großer Spannung zu spannungsärmeren Stellen verursachen [Sridhar et al., 1997a]. So zeigt sich, dass $\mathrm{Al}_{3} \mathrm{Ni}$ Stäbchen in einem gerichtet erstarrten Eutektikum $\left(\mathrm{Al}-\mathrm{Al}_{3} \mathrm{Ni}\right)$ bei $350^{\circ} \mathrm{C}$ in Kugeln zerfallen, wenn die Probe Spannungen ausgesetzt wird. Dagegen bleibt sie im spannungsfreien Zustand bis $600^{\circ} \mathrm{C}$ stabil. Ein in letzter Zeit von der theoretischen Seite verstärkt propagiertes Modell zur Beschreibung der Mikrostrukturveränderung in Vielfachschichten ist die Cahn-Hilliard-Simulation [Hentschel et al., 1998]. Dabei handelt es sich um die Lösung von Diffusionsgleichungen senkrecht zu den Lagen, die um Terme zur Berücksichtigung der Grenzflächenenergie erweitert worden sind. Entwickelt wurde sie aus Arbeiten zur spinodalen Entmischung und ist in ihrer nichtlinearen Form nicht geschlossen lösbar. Numerisch lassen sich mikrostrukturelle Veränderungen simulieren, die so weit getrieben werden können, dass sich aus einem Schichtsystem eine Lagenstruktur senkrecht dazu ausbildet, was experimentell jedoch nie beobachtet wurde. Eher simpel erscheint dagegen die Möglichkeit, dass eine Kristallisation in der Matrixschicht zu Kristalliten führen könnte, 
die die interkalierten Schichten durchstoßen und somit die Desintegration auslösen. Schließlich ist auch ein Kriechen des Matrixmetalls in die Korngrenzen der interkalierten Schicht denkbar, was dort zunächst zu Brücken zwischen den Matrixschichten und später zur Zerstörung der Schichtstruktur führen könnte. Dieser Prozess ist bei Systemen, die Kohlenstoff als Matrixmaterial beinhalten, mehrfach gezeigt worden [Bobeth et al., 1997]. In den folgenden Abschnitten sollen die einzelnen Modelle kurz vorgestellt und mit den Messergebnissen verglichen werden.

\subsection{Cahn-Hilliard-Simulationen und die Desintegration}

Die Cahn-Hilliard-Gleichung [Cahn et al, 1958] ist eine Diffusionsgleichung, in der der Grenzflächeneinfluss mit berücksichtigt wird. So wird eine quantitative Beschreibung der Phasentrennung in binären Systemen möglich, die sich bei der Beschreibung der spinodalen Entmischung bewährt hat. In ihrer nichtlinearen Form ist die Gleichung nicht analytisch lösbar, kann aber durch numerische Simulationen genährt werden [Küpper et al., 1994]. Die Gleichung der freien Energie, auf der die Rechnungen basieren, hat die folgende Form, wobei H(T) der Gradientenenergieterm und Q der Probenquerschnitt senkrecht zur x-Richtung ist:

$$
F=Q \int\left[f(c)+H\left(\frac{d c}{d x}\right)^{2}\right] d x
$$

Aus der Minimierung des freien Energieterms erhält man unter Berücksichtigung der Volumenerhaltung die eindimensionale Cahn-Hilliard-Gleichung [Haasen K12, 1994]:

$$
\frac{\partial c}{\partial t}=M(T) \cdot \nabla^{2} \frac{\partial f}{\partial c}+H(T) \cdot M(T) \cdot \nabla^{4} c
$$

Mit $\quad M=\frac{D(T)}{k T} c \cdot(1-c), H=\gamma \cdot \frac{\Omega \cdot a}{\left(c_{\beta}-c_{\alpha}\right)^{2}}$, dem Diffusionskoeffizient $\mathrm{D}$, dem Atomvolumen $\Omega$ und der Grenzflächenenergie $\gamma$. Informationen zur Programmierung solcher Simulationen finden sich in [Ewert, 1998].

Das Ergebnis solcher Simulationen ist, dass benachbarte Lagen dann ihre Gestalt verändern, wenn sie leicht unterschiedliche Lagendicken haben. So wächst beispielsweise eine ursprünglich $4 \mathrm{~nm}$ dicke Nioblage auf Kosten einer ursprünglich $3 \mathrm{~nm}$ dicken Lage, wobei beide Lagen durch $12 \mathrm{~nm}$ Kupfer voneinander getrennt 
sind. Führt man solche Simulationen in zwei Dimensionen durch, kann sich dabei nicht nur die Periodenlänge der Vielfachschicht verändern, sondern im Extremfall sogar eine Lagenstruktur senkrecht zur ursprünglichen Struktur einstellen. Obwohl diese Simulationen theoretisch sehr interessant sind, scheinen sie die die hier vorgestellten Experimente nicht beschreiben zu können. Im Experiment zeigt sich, dass die Lagenstruktur bis zum Beginn der Desintegration fast unverändert erhalten ist und die Lagendicken den ursprünglichen Werten entsprechen. Eine Verdickung einzelner Lagen aufgrund eines Diffusionsprozesses senkrecht zu den Grenzflächen konnte in keinem Fall beobachtet werden. Auch die Reduktion der Zahl der Lagen durch Auflösung dünnerer Lagen wurde in TEM-Untersuchungen nicht gefunden.

\subsection{Kristallisation der Matrixschicht und die Desintegration}

Neben Desintegrationsmechanismen, die durch treibende Kräfte verursacht durch den Einfluss der Grenzflächen erzeugt werden, kann grundsätzlich auch ein Rekristallisationsprozess zur Zerstörung der Schichtstruktur führen. Dabei kommt bei hinreichend hohen Temperaturen sowohl eine Rekristallisation im Matrixmaterial, als auch in der interkalierten Schicht in Frage.

Im Fall der Rekristallisation der nur wenige Nanometer dicken interkalierten Schicht ist die Zerstörung der Schichtstruktur unmittelbar verständlich. Rekristallisiert die Matrixschicht, führt der Prozess nur dann zu einer Desintegration, wenn die wachsenden Körner die interkalierte Schicht im Sinne einer plastischen Verformung durchstoßen. Ist die Schicht nicht mehr zusammenhängend, führen sowohl mechanische Spannungen wie auch die Grenzflächenspannung zur Reduktion der Grenzfläche zwischen den Metallen. Es bilden sich abgerundete Partikel.

Ein solcher Vorgang ist zum Beispiel im System Kobalt/Kupfer denkbar, bei dem die Desintegration erst bei über $700^{\circ} \mathrm{C}$ einsetzt. Aufgrund der hohen Temperatur ist die Kinetik hinreichend groß, um durch Bildung von Partikeln mit einem Radius von größer als der 1,5fachen Lagendicke die Grenzfläche zu reduzieren. 


\subsection{Korngrenzkriechen und die Desintegration}

Das Korngrenzkriechen beschreibt einen Prozess, bei dem ein leicht diffundierendes Matrixmaterial in die Korngrenzen einer interkalierten Schicht eindringt und auf diese Weise Brücken zwischen den Matrixlagen bildet. Ein solcher Prozess wurde z.B. im System Nickel/Kohlenstoff beobachtet, wo der Kohlenstoff in die Korngrenzen des Nickel gekrochen ist und zu einer Desintegration der Nickelschichten bei etwa $450^{\circ} \mathrm{C}$ führte [Bobeth et al., 1997]. Von den untersuchten Systemen kommt für einen solchen Vorgang nur das System Kobalt/Kohlenstoff in Frage, das jedoch bis zur maximal erreichbaren Temperatur stabil war. In der Querschnittspräparation sind ebenfalls keine Kohlenstoffbrücken durch die Kobaltlagen zu erkennen (Abbildung 3.16).

Die Beschreibung von Korngrenzkriechen in kristallinen Systemen wurde in der Literatur nicht gefunden. Die Ursache dafür ist die geringe Diffusion von Metallen im Vergleich zum amorphen Kohlenstoff in Verbindung mit der geringen treibenden Kraft des Prozesses.

\subsection{Rauigkeiten der Grenzfläche und die Desintegration}

Die folgenden Überlegungen zur Variation des chemischen Grenzflächenpotenzials durch eine lokale Variation der Grenzflächenkrümmung wurden ursprünglich zur Analyse der Gestaltinstabilität von gerichtet erstarrten eutektischen Systemen entwickelt [van Suchtelen, 1977], [Cline, 1971].

Das lokale chemische Grenzflächenpotenzial an gekrümmten Grenzflächen wird beschrieben durch die Gibbs-Thomson Relation:

$$
\mu=\mu_{0}+\frac{\gamma}{r}
$$

dabei ist $\mu$ das chemische Grenzflächenpotenzial der gekrümmten und $\mu_{0}$ das der ungestörten Grenzfläche, $\gamma$ die Grenzflächenenergie und $\mathrm{r}$ der lokale Krümmungsradius. Durch die Variation des chemischen Potenzials wird eine Diffusionsstrom $\mathbf{J}_{\text {diff }}$ erzeugt, der proportional zum Gradienten des chemischen Potenzials ist:

$$
J_{\text {diff }} \propto \operatorname{grad}(\mu)
$$

Die Diffusionsgleichung lässt sich für rotationssymmetrische Kofigurationen, z.B. 
eine Anordnung paralleler, kosinusförmig dickenmodulierter Zylinder in einer nicht mischenden Matrix, lösen.

Abhängig von $\mathrm{k} \cdot \mathrm{R}_{0}$, dem Produkt aus Wellenzahl der Dickenmodulation $\mathrm{k}$ und dem Radius des ungestörten Zylinders $\mathrm{R}_{0}$, erhält man drei Bereiche (Abbildung 5.1):

1. Für sehr langwellige Modulationen $\left(k \cdot R_{0} \rightarrow 0\right)$ ist die Grenzfläche quasi stabil, d.h. Zylinder mit konstanter Dicke zeigen keine Instabilität.

2. Für kurzwellige Modulationen $\left(k \cdot R_{0}>1\right)$ mit einer Wellenlänge kleiner als dem Umfang des ungestörten Zylinders bilden sich die Modulationen zurück, d.h. der stabile Zustand der glatten Grenzfläche wird eingenommen.

3. Für Modulationen mit Wellenlängen $0<k \cdot R_{0}<1$ vergrößert sich die Amplitude der Modulation, d.h. das System ist instabil bezüglich eines Zerfalls in Kugeln.

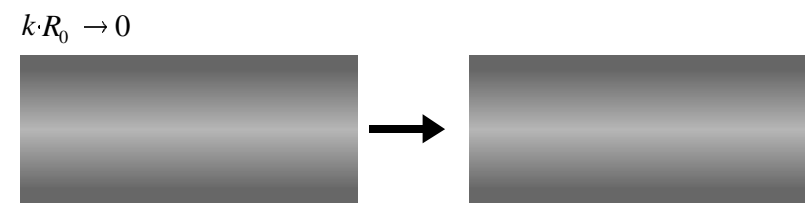

$k \cdot R_{0}>1$

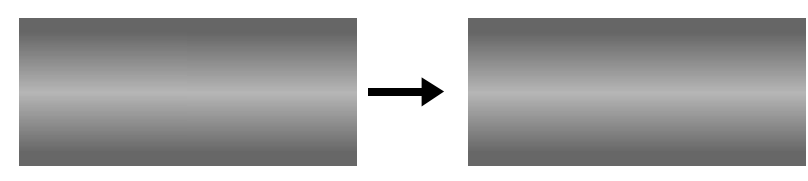

$0<k \cdot R_{0}<1$

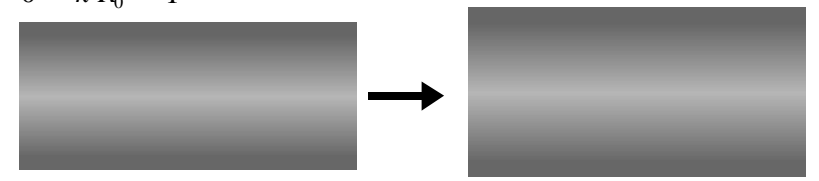

Abbildung 5.1: Eine Desintegration von dickenmodulierten Zylindern ist nur im Falle einer Rauigkeit mit einer Wellenlänge größer als dem Umfang des Zylinders zu erwarten. Im Grenzfall unendlich langer Wellenlängen ist der Zustand des Zylinders wieder stabil.

Die Geschwindigkeit der Gestaltsveränderung wird dabei in allen Fällen mit einer Abhängigkeit proportional zu $R_{0}^{-3}$ beschrieben.

Dieses Modell ist also in der Lage, die Gestaltinstabilität von Zylindern aufgrund von Variationen der Grenzflächenkrümmung über einen großen Wellenlängenbereich zu beschreiben.

In dieser Arbeit wurden aber keine stäbchenförmigen Eutektika untersucht, sondern Lagenstrukturen. Gesputterte Vielfachschichten bauen solche symmetrische Rauigkeiten, bei denen die Schicht an der oberen und unteren Grenzfläche gleichzeitig dicker oder dünner wird, nicht auf. Die Lagen legen sich viel mehr auf eine gewellte Unterlage und variieren ihre Dicke nur schwach, wie an den verschiedenen TEM-Bildern der gezeigten Proben deutlich zu erkennen ist. Des Weiteren ist in 
Lagensystemen keine geschlossene Lösung der Diffusionsgleichung mehr möglich, was dazu führt, dass eine Simulation nötig ist, um die Veränderung der Grenzfläche durch rauigkeitsinduzierte Diffusion darzustellen. Dabei wurden einige Einschränkungen gemacht. Zunächst wurde davon ausgegangen, dass nur eine Diffusion durch die Grenzflächen stattfindet, also kein Materialtransport zwischen den Lagen berücksichtigt werden muss. Des Weiteren wurde davon ausgegangen, dass die Materialflüsse von Matrix und interkalierter Schicht entgegengesetzt gleich sind, d.h., dass keine Poren in der Schichtstruktur entstehen. Außerdem wurden keine anderen Grenzflächeneffekte oder Korngrenzen berücksichtigt. Diese beiden Annahmen wurden durch die TEM-Untersuchungen gerechtfertigt. So zeigen Auswertungen der Frühstadien der Desintegration quantitativ, dass das Volumen in den einzelnen Lagen erhalten ist, eine lokale Verdickung einer Lage wird durch eine entsprechende Abnahme in der Umgebung kompensiert. Das lässt den Schluss zu, dass zumindest in den Frühstadien der Desintegration davon ausgegangen werden kann, dass der Materialtransport zwischen den Lagen keine signifikante Rolle spielt. Des Weiteren gibt es keine Anzeichen für eine Porenbildung in den ausgelagerten Schichtpaketen. Dies wird insbesondere im System Eisen/Silber deutlich. Hier existieren Partikel in den Makrokorngrenzen und innerhalb der Makrokörner, ohne dass an den Grenzflächen ein freies Volumen erkennbar wäre.

Mit diesen Randbedingungen berechnet die Simulation die Diffusion des interkalierten Metalls im Matrixmetall für eine kohärente Grenzfläche. Als treibende Kraft wird auch hier alleine der Gradient im chemischen Potenzial durch die lokal veränderliche Krümmung der Grenzfläche - entsprechend Gl. 5.3 und Gl. 5.4 - angenommen. Weitere Details zu den Diffusionsgleichungen finden sich in [Cline, 1971].

Zur Überprüfung der Simulation wurde das Programm zuerst auf einen Schnitt durch einen kosinusmodulierten Zylinder angewandt. Es stellte sich heraus, das die aus der Simulation gewonnen Ergebnisse zur Gestaltsveränderung einen ähnlichen Zustand nach der Desintegration zeigten, wie in den Berechnungen [Cline, 1971] vorhergesagt (Abbildung 5.2). Eine Anwendbarkeit der Simulation auf eine Schichtstruktur scheint somit möglich zu sein. 
Für die Simulation wurde dann eine Grenzfläche verwendet, die in eine Richtung kosinusmoduliert ist und in die andere Ebenenrichtung als konstant fortgesetzt angenommen wurde. Dadurch erhält man eine Grenzfläche, die einem Wellblech ähnelt. Lässt man bei der „Wellblechanordnung“ der Lage keine lokalen Dickenvariationen $\mathrm{zu}$, so sind die Materialströme an

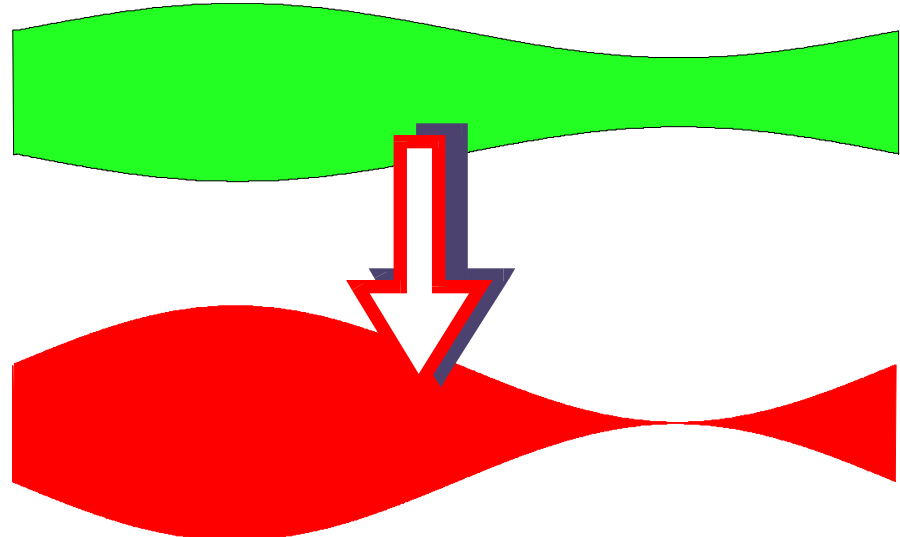

Abbildung 5.2: Simulation der Gestaltinstabilität eines kosinusmodulierten Zylinders. Oben die ursprüngliche Form, unten die Form unmittelbar vor der Desintegration. der Ober- und Unterseite der Schicht vom Betrag her gleich, aber aufgrund des unterschiedlichen Vorzeichens der Krümmung einander entgegengerichtet. Die Simulation führt zu einer Vergrößerung der Amplitude, wobei die Dicke der Lage bedingt durch die Volumenerhaltung homogen abnimmt. In der Realität würde ein solcher Vorgang nicht ablaufen können, da die Grenzflächenenergie entsprechend der vergrößerten Grenzfläche nur ansteigen würde.

Dass diese Annahme der ideal gleich dicken Lagen nicht der Realität entspricht, zeigt sich auf den TEM-Bildern, die im Rahmen der Messergebnisse vorgestellt wurden. So ist zum Beispiel im System Eisen/Gold (Abbildung 5.3) zu erkennen, dass die Schicht in der Makrokorngrenze eine deutliche Dickenvariation zeigt, während sie innerhalb der Makrokörner kaum verändert ist. Für diesen Fall sagt die Simulation keine Aufschaukelung der Grenzfläche vorher, sondern ergibt eine Desintegration der Schicht in drei Teile. Danach würden innerhalb der Makrokörner flache Scheiben der Schicht verbleiben und in den Makrokorngrenzen ein Nanopartikel gebildet, das unterhalb der Verbindungslinie der Partikel in den Makrokörnern liegt. Es muss an dieser Stelle nochmals betont werden, dass bei der Simulation keine bevorzugte Diffusion in den Korngrenzen, sondern reine Diffusion im Matrixmetall parallel zur Grenzfläche angenommen wurde. Die Vergrößerung ist an diesen Stellen allein durch die lokalen Krümmungsverhältnisse bedingt. Dieses Ergebnis erinnert stark an die Bilder der desintegrierten Eisen/Silber-Schicht (Abbildung 4.10, Seite 48), bei der ebenfalls Nanopartikel in den Makrokorngrenzen gefunden wurden, während innerhalb der Makrokörner diskusförmige Partikel 


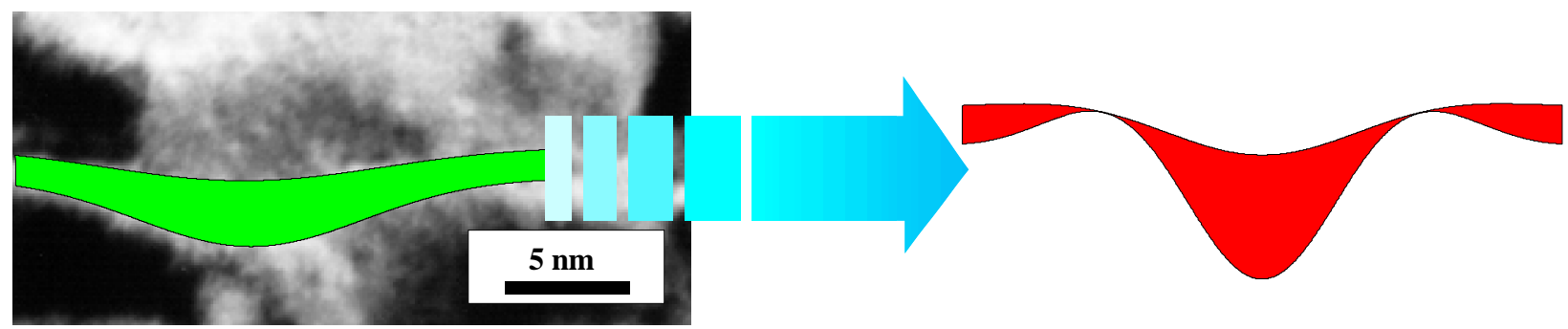

Abbildung 5.3: Simulation der Desintegration einer interkalierten Lage durch die Variation der Grenzflächenkrümmung. Dabei wurde das Profil der Ausgangsstruktur an die Grenzflächen einer Eisenlage in einem Eisen/Gold-Schichtsystem an einer Makrokorngrenze angepasst. Rechts ist die Form unmittelbar vor der Desintegration der Schicht zu sehen.

vorlagen. Auch die Lage der Partikel in den Korngrenzen, die aus der Verbindungslinie der diskusförmigen Partikel nach unten versetzt ist, wurde in den Systemen Eisen/Silber und Eisen/Gold gefunden. Im System Eisen/Gold ist ein Dreiteilung der Schicht jedoch nicht zu erkennen. Hier sind innerhalb der Makrokörner keine flachen Partikel zu finden, was durch die geringere Breite der Makrokörner in diesem System begründet sein könnte. Ein ebenfalls anders Bild erscheint nach der Desintegration der Niob/Kupfer-Schichten, da hier im wie-hergestellten Zustand eine grundlegend unterschiedliche Mikrostruktur vorlag, die weit weniger regelmäßig ist, als in den Systemen Eisen/Gold und Eisen/Silber. Aufgrund der deutlichen Krümmungen der Grenzflächen ist dieser Prozess aber auch für dieses System wahrscheinlich.

Bei all diesen Ergebnissen ist zu beachten, dass die Simulation die zweite Ebenenkoordinate vollständig außer Betracht lässt. Da der hier vorgestellte Prozess immer in Richtung der stärksten Krümmungsvariation abläuft, sind die Tripelpunkte zwischen drei Makrokörnern ganz bevorzugte Stellen, um die Nanopartikel zu bilden (Abbildung 5.4). Diese Beobachtung wurde auch experimentell gemacht und auf Seite 50 in Abbildung 4.11 schon dargestellt.

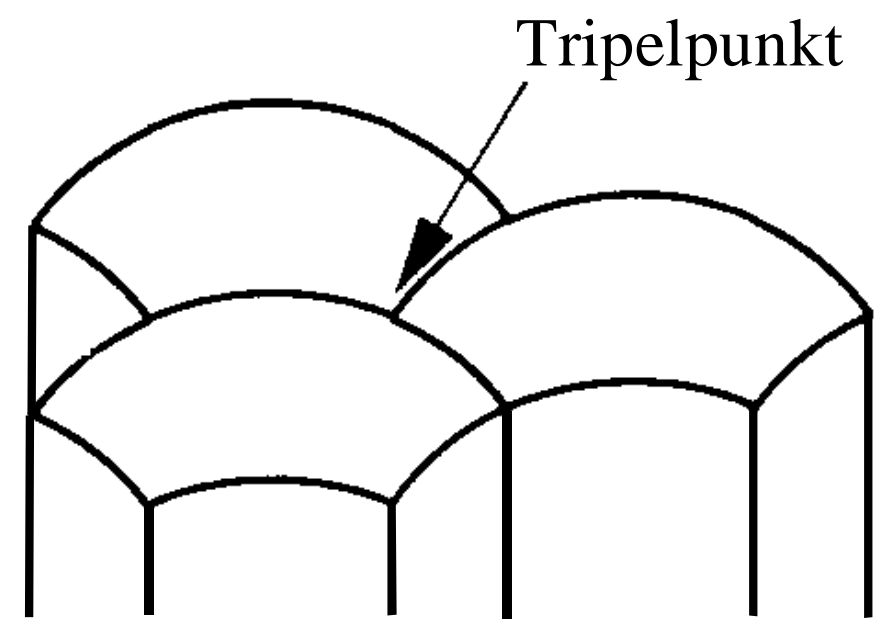

Abbildung 5.4: Krümmungsverhältnisse an gebogenen Grenzflächen der Makrokörner. 


\subsection{Mechanische Grenzflächenspannungen und die Desintegration}

Die Ergebnisse des vorherigen Kapitels legen es nahe, dass die Desintegration der Schichtstruktur in den Systemen Niob/Kupfer und Eisen/Silber durch die lokale Variationen der Grenzflächenkrümmung in Verbindung mit Variationen der Lagendicken bedingt ist. Dabei wurde ein wichtiger Aspekt, der die Energie der Grenzfläche stark beeinflusst, noch nicht mit in die Überlegung einbezogen, nämlich die Spannungen an Grenzflächen. Dieser Aspekt scheint um so wichtiger, da das Krümmungsmodell alleine die Mikrostruktur des Systems Eisen/Gold nach der Auslagerung nicht erklären kann.

Gradienten im chemischen Potenzial können aber auch an planaren Grenzflächen auftreten, wenn zum Beispiel die durch epitaktisches Wachstum bedingte Spannung an Korngrenzen senkrecht zur Schichtstruktur relaxieren. In dünnen Schichten muss dabei neben den Volumenspannungen auch der Beitrag der Grenzflächenspannungen berücksichtigt werden. Die Veränderung der Energie im Schichtsystem ergibt sich aus der Summe der beiden Beiträge:

$$
\Delta U=\Delta U_{\text {Volumen }}+\Delta U_{\text {Grenzfl. }}
$$

Nach [Streitz et al., 1994] führt der Einfluss der Grenzfläche zu einer effektiven Veränderung der Dehnung $\epsilon^{*}$ an den Grenzflächen. Zu deren Bestimmung minimiert er die Energiebeiträge von Volumen- und Grenzflächenspannung bezüglich $\epsilon$. Die Ausdrücke für die Veränderung der Volumenenergie und der Grenzflächenenergie sind:

$$
\begin{aligned}
& \Delta U_{\text {Volumen }}=V(\epsilon) \sum_{i, j} \int \sigma_{i j} d \epsilon_{i j}=2 V(\epsilon) \int \sigma(\epsilon) d \epsilon=2 V(\epsilon) \iint Y\left(\epsilon^{\prime}\right) d \epsilon^{\prime} d \epsilon \\
& \Delta U_{\text {Grenzfl. }}=2 \int f(\epsilon) d A=2 \int f(\epsilon) \frac{d A}{d \epsilon} d \epsilon=4 \int f(\epsilon) A_{0}(1+\epsilon) d \epsilon
\end{aligned}
$$

Dabei wird vorausgesetzt, dass sowohl Spannungen als auch Dehnungen in der Filmebene isotrop sind und dass der effektive elastische Modul $Y$ und die Grenzflächenspannung $f$ von der Dehnung abhängig sind. Der Volumenausdruck in Gl. 5.6 setzt sich aus dem dehnungsabhängigen Volumen der Lage $V(\epsilon)=\lambda(\epsilon) \cdot A(\epsilon)$ und der Spannungsenergie zusammen. Der Grenzflächenterm ist das Produkt aus der dehnungsabhängigen Grenzflächenenergie und der Fläche $A$. Entwickelt man den elastischen Modul $Y$ und die Grenzflächenenergie $f$ gemäß $Y(\epsilon) \approx Y_{0}\left(1-Y_{1} \epsilon\right)$ bzw. $f(\epsilon) \approx f_{0}+f_{1} \epsilon$, so ergibt sich: 


$$
\frac{\Delta U}{2 A_{0}} \approx 2 f_{0} \epsilon+\left(f_{0}+f_{1}+\frac{1}{2} \lambda_{0} Y_{0}\right) \epsilon^{2} \approx 2 f_{0} \epsilon+\frac{1}{2} \lambda_{0} Y_{0} \epsilon^{2}
$$

Wobei $\lambda_{0}$ die Periodenlänge des ungedehnten Schichtpaketes ist und über das Volumen in den Ausdruck eingeführt wird. Nach [Streitz et al., 1994] können folgende typische Werte gefunden werden: $\lambda_{0} \mathrm{Y}_{\infty} \sim 15 \mathrm{eV} / \AA^{2}, \mathrm{f}_{0} \sim 0,05 \mathrm{eV} / \AA^{2}$ und $\mathrm{f}_{1} \sim 0,3 \mathrm{eV} / \AA^{2}$.

Dann folgt:

$$
\epsilon^{*} \approx-\frac{2 f_{0}}{\lambda_{0} Y_{0}} \quad \text { bzw. } \quad \frac{\Delta U}{2 A_{0}} \approx-\frac{2 f_{0}^{2}}{\lambda_{0} Y_{0}}
$$

Dieses Ergebnis ist wie folgt $\mathrm{zu}$ interpretieren. In einem kohärent verspannten Schichtpaket nimmt der Einfluss der Spannungen auf die Grenzflächenenergie mit abnehmender Periodenlänge zu. Die treibenden Kräfte infolge von Spannungsgradienten zwischen dem Inneren der Makrokörner und den Korngrenzen vergrößern sich entsprechend und zwar in Übereinstimmung mit den Experimenten, die ein Abfallen der Desintegrationstemperatur mit 1/d zeigen. Diese Interpretation der reziproken Abhängigkeit der Desintegrationstemperatur von der Periodenlänge impliziert aber auch einen Übergang von den hier vorausgesetzten kohärenten zu semikohärenten Grenzflächen ab der Lagendicke bei der die Desintegrationstemperatur deutlich zunimmt.

Die Desintegrationsgeschwindigkeit wurde unter Berücksichtigung beider Beiträge, Grenzflächenkrümmung und Spannung, von Sridhar [Sridhar et al., 1997b] berechnet. Auf eine Darstellung dieser Rechnungen wird hier verzichtet, da sie keine konsequente Lösung der Diffusionsgleichungen bieten, sondern nur die entsprechenden Energieterme abschätzen. Das führt zum Beispiel dazu, dass Systeme mit sehr großen Grenzflächenenergien grundsätzlich nicht zerfallen, was im Widerspruch zu den entsprechenden Rechnungen von Cline steht. Trotzdem liefern die Überlegungen von Sridhar zwei wesentliche Aussagen. Die Desintegrationsgeschwindigkeit nimmt mit zunehmender Verspannung der Lagen und zunehmender Differenz der elastischen Module der Lagen zu. Diese Effekte werden durch die zwei Parameter $\Theta$ und $\alpha$ beschrieben:

$$
\Theta:=\frac{\gamma}{E_{A} \lambda \epsilon^{2}} \quad \text { und } \quad \alpha:=\frac{E_{A}-E_{B}}{E_{A}+E_{B}}
$$

Dabei ist $\mathrm{E}_{\mathrm{A}}$ der elastische Modul der interkalierten Schicht und $\mathrm{E}_{\mathrm{B}}$ der der Ma- 
trixschicht. Systeme, die zu einer schnellen Desintegration neigen, sollten danach durch einen kleinen Wert von $\Theta$ und einen Wert nahe 1 von $\alpha$ gekennzeichnet sein. Diese Parameter erscheinen auch physikalisch durchaus plausibel. Bei gegebener Fehlpassung sind die Spannungsenergien in den elastisch härteren Lagen größer. Während die Grenzflächenenergie im wesentlichen die Tendenz zur Minimierung der Grenzfläche unter Beibehaltung der Kohärenz beschreibt, werden große Spannungsenergien zu einer Aufgabe der Kohärenz führen. Diese Parameter werden bei der Diskussion der treibenden Kräfte wieder aufgegriffen. 


\section{Grenzflächenenergien der Schichtsysteme}

Nachdem im vorherigen Kapitel die Veränderungen der Grenzflächenenergie aufgrund von Krümmungs- und Spannungseinflüssen an den Grenzflächen diskutiert wurden, soll das Augenmerk in diesem Abschnitt auf die Energie der inneren Metall-Metall-Grenzflächen bzw. Metall-Halbmetall-Grenzflächen gelenkt werden. Die Bedeutung der Grenzflächen in den Vielfachschichten nimmt mit abnehmender Periodenlänge $\mathrm{zu}$, da ein immer größerer Anteil der Atome im Einflussbereich der Grenzflächen liegt. Ein gutes Beispiel dafür ist die Hochtemperaturphase des Kobalt, die in den Vielfachschichten durch den Einfluss der Grenzflächen bei Raumtemperatur stabilisiert wird.

Die Energie einer Grenzfläche setzt sich dabei im wesentlichen aus einem chemischen Beitrag der beteiligten Metalle $\gamma_{\text {chem }}$ und einem strukturellen Anteil $\gamma_{\text {elast }} \mathbf{z u}$ sammen. Für den strukturellen Teil der Grenzflächenenergie ist die Art der Grenzfläche von großer Bedeutung. Man unterscheidet zwischen kristallin-amorphen Grenzflächen, inkohärenten und semikohärenten Grenzflächen, kohärenten Grenzflächen und epitaktischen Grenzflächen.

\subsection{Der chemische Anteil der Grenzflächenenergie}

Die Grenzflächenenergie zweier Materialien ist allein durch den chemischen Beitrag bestimmt, wenn die beiden kohärent und ohne Fehlpassung aufwachsen bzw. wenn die eine Komponente amorph ist. Für den ersten Fall kann die Grenzflächenenergie nach [Becker, 1938] aus der Energie der gleichnamigen Bindungen, deren Dichte und der Paarvertauschungsenergie berechnet werden. Sind die Atome statistisch verteilt und ist der Anteil der B-Atome gleich c, erhält man für die Enthalpie folgende Gleichung:

$$
\tilde{h}=\frac{1}{2}\left[(1-c) \epsilon_{A A}+c \epsilon_{B B}+2 c(1-c) \epsilon\right]
$$

Die Paarvertauschungsenergie $\epsilon$ kann dabei nach [Becker, 1938] aus der Krümmung der Exzess-Enthalpie gewonnen werden. Für Systeme mit unterschiedlichen Randlöslichkeiten kann das Modell nach [Borchers et al., 2000] entsprechend erweitert werden. 
Mit Hilfe der thermodynamischen Daten von [Swartzendruber, 1984], [Okamoto, 1984] und [Borchers et al., 2000] lässt sich danach die Grenzflächenenergie von dichtest gepackten Ebenen für das System Kobalt/Kupfer zu $\gamma_{\text {chem }}=185 \mathrm{~mJ} / \mathrm{m}^{2}$, für das System Eisen/Silber $\mathrm{zu} \gamma_{\text {chem }}=680 \mathrm{~mJ} / \mathrm{m}^{2}$, für das System Eisen $/$ Gold $\mathrm{zu}$ $\gamma_{\text {chem }}=80 \mathrm{~mJ} / \mathrm{cm}^{2}$ und für das System Niob/Kupfer zu $\gamma_{\text {chem }}=540 \mathrm{~mJ} / \mathrm{m}^{2}$ bestimmen. Der Wert für das System Niob/Kupfer gilt dabei für den Fall der kohärenten Grenzfläche. Im Fall inkohärenter Grenzflächen ist diese Abschätzung aufgrund der veränderten Bindungsverhältnisse an der Grenzfläche eher als obere Grenze des chemischen Beitrags zur Grenzflächenenergie zu sehen. Experimentell findet man im System Kobalt/Kupfer eine Grenzflächenenergie von $\gamma_{\text {chem }}=200-265 \mathrm{~mJ} / \mathrm{m}^{2}$ [Hattenhauer et al., 1993], [Hattenhauer, 1992], [Shiftlet et al., 1981], [Servi et al., 1966]. Der geringe chemische Beitrag im System Eisen/Gold im Vergleich zum Eisen/Silber resultiert daraus, dass in diesem System eine gewisse Randlöslichkeit auftritt, was eine kleinere repulsive Wechselwirkung zwischen Eisen und Gold zeigt.

Bei einer kristallin-amorphen-Grenzfläche trifft ein geordneter Festkörper auf einen ungeordneten, glasförmigen Festkörper. In diesem Fall ist die Voraussetzung des Modells von Becker nicht mehr gegeben [Borchers et al., 1999]. Eine grobe Abschätzung ergibt sich aus den Oberflächenspannungen der beteiligten Komponenten, da nach [Bilaniuk et al., 1998] gilt:

$$
\gamma_{C o} \gg \gamma_{C o / C}>\gamma_{C}
$$

G1. 6.2

Damit lässt sich die Grenzflächenenergie auf den Bereich von $\gamma_{\mathrm{Co}} \approx 2700 \mathrm{~mJ} / \mathrm{m}^{2}$ [Mezey et al., 1982] und $\gamma_{\mathrm{C}} \approx 40 \mathrm{~mJ} / \mathrm{m}^{2}$ im amorphen Kohlenstoff grob einschränken.

\subsection{Der strukturelle Anteil der Grenzflächenenergie}

\subsubsection{Inkohärente Grenzflächen}

Eine inkohärente Grenzfläche ist das Analogon zur Großwinkelkorngrenze innerhalb einer Phase. Zwischen den an diesen Grenzflächen aufeinander treffenden Kristalliten besteht keine konkrete Orientierungsbeziehung wie es bei kohärenten oder semikohärenten Grenzflächen der Fall ist. Zur Minimierung der Energie bilden 


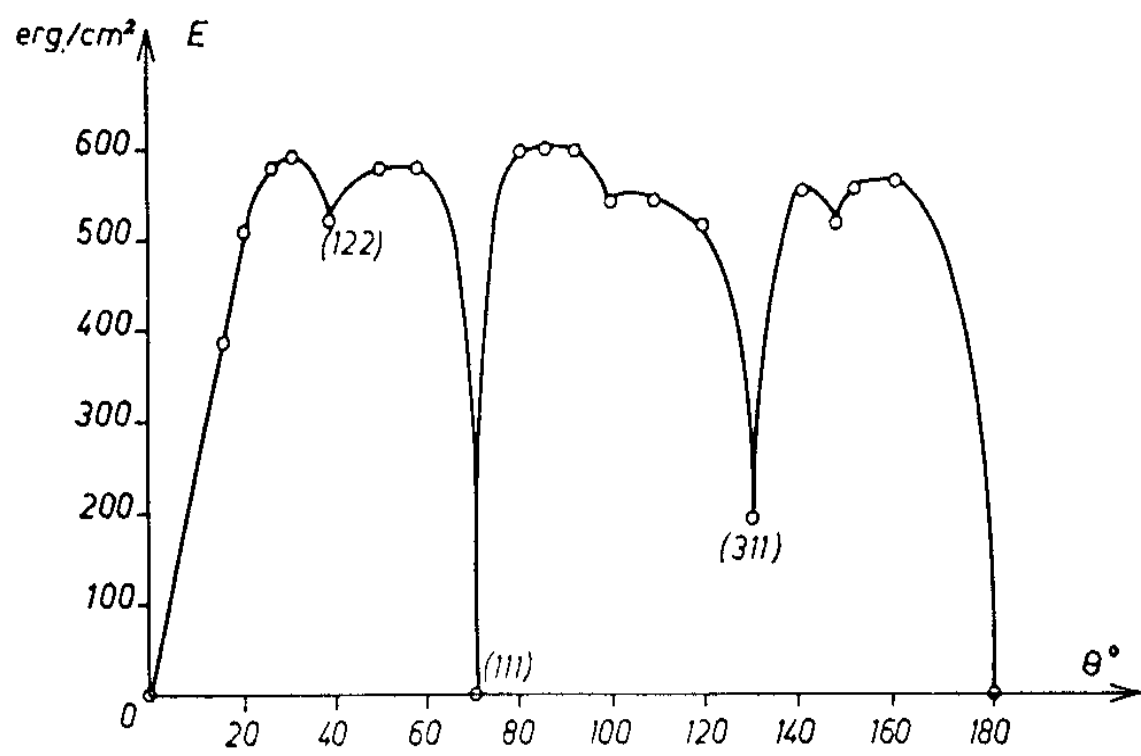

Abbildung 6.1: Energie einer inkohärenten Grenzfläche bzw. einer Großwinkelkorngrenze in Abhängigkeit des Kippwinkels zwischen den Körnern [Hasson et al., 1972].

sich bei bestimmten Orientierungen der Körner an den Grenzflächen Struktureinheiten aus, die sich regelmäßig wiederholen. In diesem Fall definiert man ein Koinzidenzgitter, das die Gesamtheit der Gitterplätze darstellt, die beiden Körnern gemeinsam ist, wenn man ihre Atomanordnung über die Korngrenze hinweg ineinander fortsetzt. Der Anteil $1 / \Sigma$ gibt den Anteil der gemeinsamen Gitterplätze an. Um so kleiner $\Sigma$ dabei ist, desto geringer ist die Energie der Korngrenze (Abbildung 6.1). Für einen konkreten Winkel lässt sich die Struktur der Korngrenze und die damit verbundene Energie nur mit viel Aufwand über molekulardynamische Simulationen ermitteln. Der Abbildung 6.1 entnimmt man aber, dass abgesehen von einigen Korngrenzwinkeln mit großer Koinzidenzdichte die Energie einer Großwinkelkorngrenze annähernd konstant ist [Hasson et al., 1972]. Eine Abschätzung für die Energie der inkohärenten Grenzfläche ergibt daher einen Wert von ungefähr $1000 \mathrm{~mJ} / \mathrm{m}^{2}$. Da in polykristallinen Filmen nicht anzunehmen ist, dass stetig Orientierungsbeziehungen mit kleinem $\Sigma \mathrm{zu}$ finden sind, wird diese Abschätzung den folgenden Überlegungen zugrunde gelegt. 


\subsubsection{Kohärente und semikohärente Grenzflächen}

Ein typisches Beispiel für eine kohärente Grenzfläche findet man im System Kobalt/Kupfer, da die Metalle mit gleicher Gitterstruktur aufeinander aufwachsen und dabei fast gleiche Atomgrößen haben. Aber auch in den hier untersuchten Systemen Eisen/Gold und Eisen/Silber wurden innerhalb der Makrokörner solche kohärenten Grenzflächen gefunden, die dann aufgrund der Nishiyama-Wassermann-Beziehung eine große Fehlpassung aufweisen. Diese Fehlpassungen erzeugen große Spannungen und damit einen großen strukturellen Beitrag zur Grenzflächenenergie, der durch den Einbau von Fehlpassungsversetzungen, d.h. dem Übergang zu einer semikohärenten Grenzfläche, effektiv verringert werden kann. Allerdings können solche Versetzungen erst ab einer kritischen Lagendicke gebildet werden, was folgende Überlegungen zeigen.

In solchen Schichtpaketen hat man wieder einen chemischen Beitrag zur Grenzflächenenergie, einen strukturellen Anteil, verursacht durch die Fehlpassung und in semikohärenten Systemen zusätzlich einen Versetzungsbeitrag. Der Beitrag der Grenzflächenenergie durch Versetzungen lässt sich aus der Energie der Versetzungen und ihrem mittleren Abstand berechnen. Dabei ist die Energie von Stufenversetzungen im Abstand d [Haasen K12, 1994]:

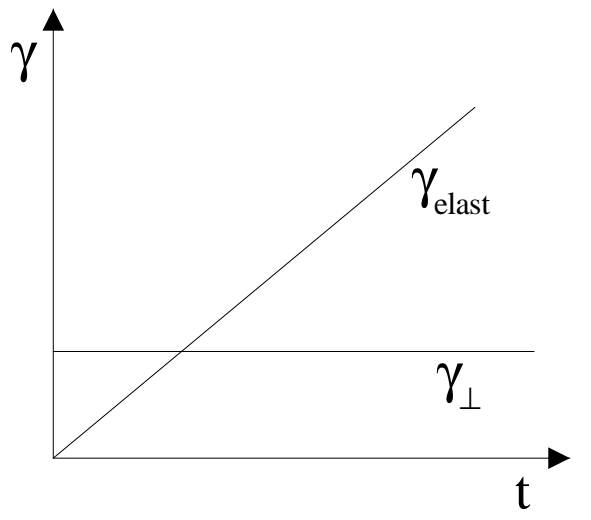

Abbildung 6.2: Abhängigkeit der elastischen und Versetzungsenergie von der Schichtdicke.

$$
\gamma_{\perp}=\frac{\mu b d}{4 \pi(1-v)} \ln \left(\frac{d}{b}-1\right)
$$

mit dem Burgersvektor $\mathrm{b}$, der Poissonzahl $\nu$ und dem Schermodul $\mu$.

Der strukturelle Beitrag zur Grenzflächenspannung ist nach [van der Merwe, 1972]:

$$
\gamma_{\text {elast }}=2 \mu \frac{(1+v)}{(1-v)} \epsilon^{2} t
$$

mit der Restverzerrung $\epsilon$ und der Schichtdicke t. Da der Energiebeitrag der Versetzungen unabhängig, der elastische Teil jedoch direkt proportional zur Schichtdicke ist, bilden sich Versetzungen erst ab einer gewissen Dicke, was anhand von Abbildung 6.2 deutlich wird. Nach [Ariosa et al., 1988] lässt sich diese Schichtdi- 
cke aus dem kritischen Radius eines Versetzungsrings $r_{C}$ abschätzen. Es muss allerdings berücksichtigt werden, dass gegebenenfalls auch andere Versetzungsformen in geringfügig dünneren Schichten auftreten können. Im Fall des Versetzungsrings ist $r_{C}$ definiert als der Radius, bei der die Selbstenergie des Versetzungsrings kleiner als die Reduktion der Spannungsenergie durch den Einbau von Versetzungen ist.

$$
r_{c}=\frac{3 b}{4 \sqrt{6} \kappa \pi \epsilon_{0}} \frac{(2-v)}{(1+v)} \ln \left(\frac{24 r_{c}}{b e}\right)
$$

Dabei ist $\nu$ die Poissonzahl $\mu$ der Schermodul, b der Betrag des Burgersvektors, e die Basis des natürlichen Logarithmus, $\kappa=\frac{1+v}{1-v}$ und $\epsilon_{0}$ die Dehnung, die zum Einbau von Versetzungen geführt hat. In folgender Tabelle sind die Werte für die Metalle Niob, Eisen, Gold und Silber [Smithells], sowie die daraus resultierenden kritischen Radien der Versetzungsringe angegeben. Die kritische Schichtdicke für den Einbau von Versetzungen kann dann als der doppelte kritische Radius des Versetzungsrings angenommen werden.

\begin{tabular}{|c|c|c|c|c|}
\hline & Eisen & Niob & Gold & Silber \\
\hline $\mathrm{b}$ & 0,1655 & 0,1906 & 0,2884 & 0,2889 \\
\hline$\nu$ & 0,293 & 0,397 & 0,42 & 0,367 \\
\hline$\epsilon_{0}$ & 0,1 & 0,2 & 0,1 & 0,1 \\
\hline $\mathrm{r}_{\mathrm{C}}[\mathrm{nm}]$ & 1,5 & 1 & 1,6 & 1,8 \\
\hline \hline
\end{tabular}

Tabelle 6.1: Kritische Radien für den Einbau von Versetzungsringen in eine dünne Schicht für verschiedene Metalle.

Nach diesen Abschätzungen sind für die hauptsächlich untersuchten interkalierten Metalle Eisen und Niob Versetzungen erst ab etwa 3 bzw. $2 \mathrm{~nm}$ zu erwarten. Dieser Bereich ist in den Messungen der Desintegrationstemperatur in Abhängigkeit von der Lagendicke abgedeckt. Im System Niob/Kupfer findet man bei 1,5 nm ein Abknicken der Kurve vom reziproken Verlauf der Desintegrationstemperatur , bei Eisen/Gold erst ab 2,5 nm. Es erhärtet sich daher die Vermutung aus Kapitel 5.5, dass dieser Knick durch den Übergang von kohärenten zu semikohärenten Grenzflächen verursacht wird. Die dadurch verringerte Spannungsenergie in den Grenzflächen führt zu einer Erhöhung der Desintegrationstemperatur aufgrund von verringerten 
treibenden Kräften.

Kohärentes Wachstums kann also erhebliche mechanische Spannungen an der Grenzfläche zur Folge haben. Wie bereits diskutiert gehorcht die Orientierungsbeziehung zwischen Gold bzw. Silber und Eisen der Nishiyama-Wassermann-Beziehung. Die Dehnung der Eisen (110)-Lage ist in diesem Fall stark anisotrop. Die Dehnung in $\langle 100\rangle_{\mathrm{krz}}$-Richtung, parallel zur $\langle 110\rangle_{\mathrm{kfz}}$-Richtung beträgt $\epsilon_{\mathrm{x}}=0,0035$, in $\langle 011\rangle_{\mathrm{krz}}$-Richtung, parallel zur $\langle 11-2\rangle_{\mathrm{kfz}}$-Richtung, beträgt sie $\epsilon_{\mathrm{y}}=0,185$ (siehe auch Abbildung 2.2, Seite 9). Die homogene Spannungsenergiedichte wird beschrieben durch:

$$
e_{\text {hom }}=2 \mu \frac{1+v}{1-v} \epsilon^{2}
$$

Der Schermodul von Fe beträgt $\mu_{\mathrm{Fe}}=11,07 \cdot 10^{10} \mathrm{~J} / \mathrm{m}^{3}$ [Landolt-Börnstein] und ist damit etwa dreimal größer als der Schermodul von Gold mit $\mu_{\mathrm{Au}}=4,23 \cdot 10^{10} \mathrm{~J} / \mathrm{m}^{3}$ [Landolt-Börnstein]. Man kann daher davon ausgehen, dass die Spannungen hauptsächlich zu einer Dehnung in den Goldschichten führen und dass damit eine Spannungsenergiedichte von $\sim 2 \cdot 10^{6} \mathrm{~J} / \mathrm{m}^{3}$ in $\langle 110\rangle_{\mathrm{kfz}}$-Richtung bzw. von $\sim 5,810^{9} \mathrm{~J} / \mathrm{m}^{3}$ in $\langle 112\rangle_{\mathrm{kfz}}$-Richtung verbunden ist. Die immensen Spannungen in den Eisen/Gold-Vielfachschichten zeigen auch die Spannungskontraste in den TEMBildern, die im Abschnitt über die Mikrostruktur (3.2) bereits diskutiert wurden (Abbildung 3.12, Seite 31). Dort wurde auch die zum partiellen Abbau der Spannungen gebildeten Welligkeit der Grenzflächen vorgestellt.

Grundsätzlich sollten die Spannungen im System Eisen/Silber ähnliche Werte erreichen, da auch in diesem System ein Übertrag der Orientierung von einer Silberlage zur nächsten über die Eisenlage hinweg beobachtet wird. Da Messungen des magnetischen Moments der Proben auch hier zeigen, dass die Eisenschichten kubisch-raumzentriert sind, kann der Übertrag über die Nishiyama-Wassermann-Beziehung geschehen. Im TEM sind jedoch neben Spannungskontrasten auch zahlreiche Zwillingsgrenzen erkennbar (Abbildung 6.3), die zum teilweisen Abbau der Spannungen führen können. Während also im System Eisen/Gold die Ausbildung der Welligkeit der wesentliche Mechanismus zur Reduktion der Spannungen zu sein scheint, werden diese in Eisen/Silber partiell zusätzlich durch Zwillingsbildung re- 
duziert. Es ist daher auch verständlich, warum bei gleicher Fehlpassung die Wellenlänge der Rauigkeiten im System Eisen/Silber deutlich größer ist.

Die Spannungsenergiedichte E von ca. $610^{9} \mathrm{~J} / \mathrm{m}^{3}$ korrespondiert $\mathrm{zu}$ einem Grenzflächenbeitrag von $1 / 2 \cdot d_{\text {Gold }} \cdot E \approx 30 \mathrm{~J} / \mathrm{m}^{2}$. Im System Eisen/Silber ist der Beitrag aufgrund der ähnlichen Fehlpassung und ähnlicher elastischer Konstanten ähnlich. In beiden Systemen ist jedoch ein nicht näher bestimmbarer Teil der Energie durch die Ausbildung von Grenzflächenkrümmungen und die Einführung von Zwillingsgrenzen reduziert. Zwillingsgrenzen können die elastische Energie je nach Dichte auf 30-50\% reduzieren. Die deutliche Reduktion der Spannungsenergie im Bereich vieler Zwillingsgrenzen lässt sich dort auch durch die veränderten Krümmungsverhältnisse der Grenzfläche erkennen (Abbildung 6.3).

Im System Niob/Kupfer liegen für Nioblagendicken unterhalb von 1,5 nm ebenfalls lokal kohärente Grenzflächen vor. Die Energie pro Fläche wird aufgrund der großen Fehlpassung von über $20 \%$ ebenfalls im Bereich mehrerer $10 \mathrm{~J} / \mathrm{m}^{2}$ liegen. Aufgrund der kleinen Kupferkörner werden sich große Dehnungen jedoch nur in kleinen Bereichen ausbilden können, so dass die Energie auch im partiell kohärenten Bereich deutlich unterhalb der der Systeme Eisen/Gold und Eisen/Silber liegen dürfte.

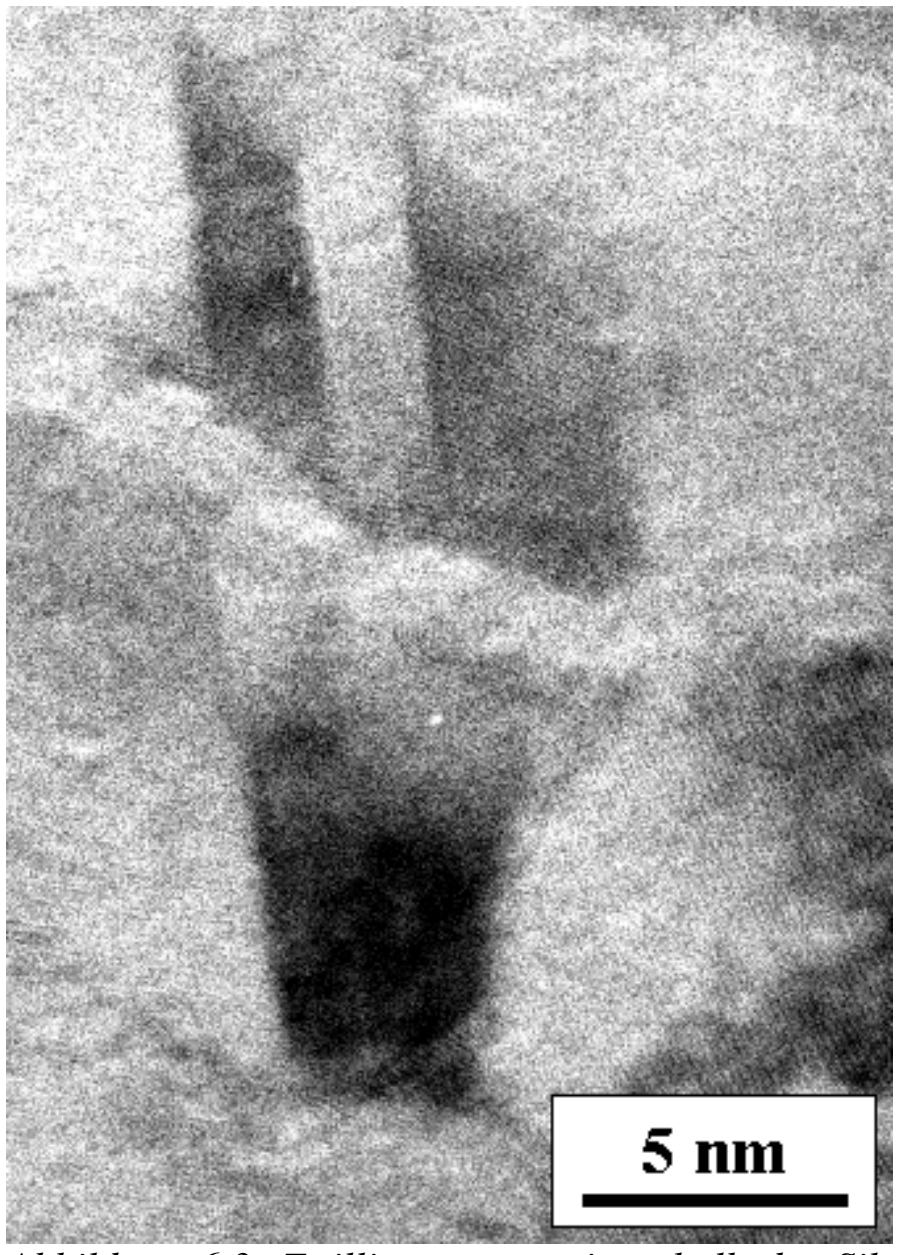

Abbildung 6.3: Zwillingsgrenzen innerhalb der Silberlagen einer Eisen/Silber-Vielfachschicht $(2 \mathrm{~nm} / 10 \mathrm{~nm})$. Sie reduzieren die Spannungsenergie im System. 


\subsection{Vergleich der Grenzflächenenergien}

Auf der Basis der vorherigen Abschnitte wird deutlich, dass die Quantifizierung von Grenzflächenenergien nicht idealer Grenzflächen nur grob möglich ist. Eine Abschätzung und ein Vergleich der Grenzflächenenergien der verschiedenen Systeme ist jedoch möglich.

Für das kohärente Schichtsystem Kobalt/Kupfer ist die Grenzflächenenergie allein durch den chemischen Beitrag von ca. $200 \mathrm{~mJ} / \mathrm{m}^{2}$ bestimmt. Die Schichten wachsen mit einer kolumnaren Struktur ohne die für die Systeme Eisen/Gold und Eisen/Silber charakteristische Welligkeit auf. Auch für Kobalt/Kohlenstoff ist die Grenzflächenenergie allein durch den chemischen Beitrag bestimmt. Eine Abschätzung ist hier kaum möglich. Die im Röntgendiffraktogramm sichtbaren Überstrukturen und die im TEM beobachtete Glätte der Schichten deutet aber auf ein ausgeprägtes Lagenwachstum hin, so dass die Grenzflächenenergie vergleichbar oder kleiner als die von Kobalt/Kupfer angenommen werden kann. Diese Annahmen stimmen nur, wenn der Kohlenstoff amorph ist. So steigt die Oberflächenspannung von Kohlenstoff durch die Kristallisation um eine Größenordnung an. Außerdem erhält die Grenzfläche in diesem Fall zusätzlich einen strukturellen Energiebeitrag.

Im System Niob/Kupfer sind die Energiebeiträge dagegen deutlich vergrößert. Hier kommt zu dem chemischen Beitrag, dessen Energie kleiner oder gleich $540 \mathrm{~mJ} / \mathrm{m}^{2}$ abgeschätzt wurde, der strukturelle Beitrag der inkohärenten Grenzfläche mit etwa $1000 \mathrm{~mJ} / \mathrm{m}^{2}$ hinzu. Die gesamte Grenzflächenenergie beträgt demnach etwa $1,5 \mathrm{~J} / \mathrm{m}^{2}$. Im Falle der partiell kohärenten Grenzflächen in sehr dünnen Nioblagen liegt die Grenzflächenenergie um einen nicht quantifizierbaren Betrag über diesem Wert. Aufgrund der kleinen Kupferkörner und der dadurch geringen effektiven Flächen kohärenten Wachstums, wird die Energie jedoch deutlich unterhalb der in den Systemen Eisen/Gold und Eisen/Silber liegen.

Die größten Grenzflächenenergien weisen die Systeme Eisen/Gold und Eisen/Silber auf. Während die strukturellen Energien aufgrund der sehr ähnlichen Gitterparameter und der in beiden Fällen vorhandenen kohärenten Grenzflächen mit Nishiyama-Wassermann-Beziehung mit $30 \mathrm{~J} / \mathrm{m}^{2}$ fast gleich sind, unterscheiden sich die Systeme aufgrund ihrer Randlöslichkeit in den chemischen Beiträgen. Hier dominiert also der strukturelle Beitrag, auch wenn er in beiden Systemen durch die 
Welligkeit und im System Eisen/Silber zusätzlich durch den Einbau von Zwillingsgrenzen reduziert wird. Nach dem Überschreiten der kritischen Lagendicke für den Einbau von Versetzungen und dem damit verbundenen Übergang zu semikohärenten bzw. inkohärenten Grenzflächen ist die Spannungsenergie in den Systemen natürlich deutlich reduziert und der strukturelle Beitrag zur Grenzflächenenergie liegt im Bereich von $1 \mathrm{~J} / \mathrm{m}^{2}$.

Zur besseren Übersichtlichkeit sind die Werte in Tabelle 6.2 nochmals zusammengefasst.

\begin{tabular}{|l|c|}
\hline \multicolumn{1}{|c|}{ System } & Grenzflächenenergie \\
\hline Kobalt/Kohlenstoff & klein \\
\hline Kobalt/Kupfer & $\sim 0,2 \mathrm{~J} / \mathrm{m}^{2}$ \\
\hline Niob/Kupfer inkohärent & $\sim 1,5 \mathrm{~J} / \mathrm{m}^{2}$ \\
\hline Eisen/Silber kohärent & $<10-20 \mathrm{~J} / \mathrm{m}^{2}$ \\
\hline Eisen/Gold kohärent & $<30 \mathrm{~J} / \mathrm{m}^{2}$ \\
\hline
\end{tabular}

Tabelle 6.2: Übersicht über die Grenzflächenenergien der verschiedenen Vielfachschichtsysteme. 


\section{Diskussion der Ergebnisse}

Nachdem die Experimente zur Desintegration von Vielfachschichten präsentiert und mögliche Mechanismen zur Desintegration dargestellt wurden, bleibt zu prüfen, inwieweit diese Modelle und Simulationen in der Lage sind, die Experimente zur Entwicklung der Mikrostruktur während der Auslagerung zu beschreiben. Es muss geklärt werden, welche Mechanismen während des Zerfalls in den verschiedenen Systemen zum Tragen kommen. Dabei muss durchaus davon ausgegangen werden, dass auch mehrere Mechanismen an der Desintegration beteiligt sein können. Insbesondere blieb bisher ungeklärt, wie die Glättung der Grenzflächen während der Desintegration vonstatten geht und wodurch die stark unterschiedlichen Desintegrationstemperaturen der Systeme zustande kommen.

\subsection{Mikrostruktur und Thermodynamik}

Bereits in der Darstellung der treibenden Kräfte wurde festgestellt, dass für die Systeme Niob/Kupfer, Eisen/Gold und Eisen/Silber, die sich durch eine Zerfallstemperatur deutlich unterhalb von $600^{\circ} \mathrm{C}$ auszeichnen, als treibende Kräfte nur die lokale Variation der Grenzflächenkrümmung und die Variation der mechanischen Spannung der Grenzflächen in Frage kommen. Nur diese Mechanismen führen zu einer Desintegration durch die experimentell beobachtete Diffusion parallel zu den Grenzflächen. Sind an den Grenzflächen sowohl Variationen in den Krümmungen, als auch in den Spannungen vorhanden, wie es teilweise beobachtet wurde, sind beide Mechanismen vertreten. Es stellt sich dann also die Frage, welcher Mechanismus wann dominiert.

Die strukturellen Beiträge der Grenzflächenenergien im Fall von kohärenten Grenzflächen mit großer Fehlpassung sind sehr groß, in Kapitel 6 wurden Energien von mehreren $10 \mathrm{~J} / \mathrm{m}^{2}$ abgeschätzt. Zwischen den kohärenten Bereichen der Grenzfläche (I in Abbildung 7.1) und den inkohä-

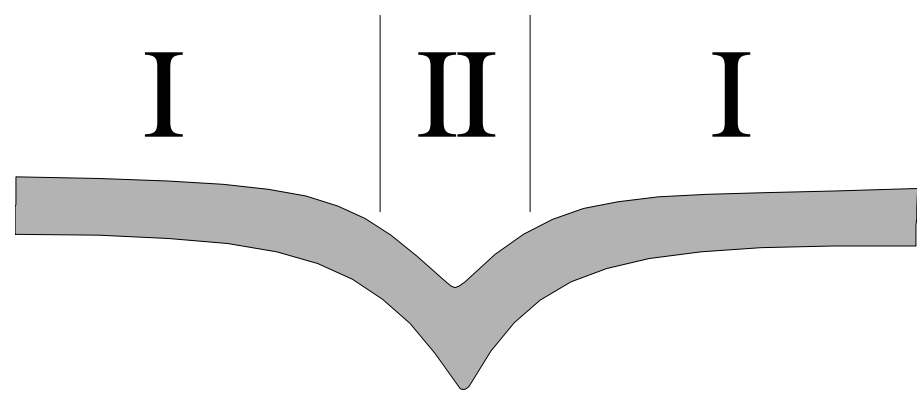

Abbildung 7.1: Schematische Darstellung einer Grenzfläche, wie sie in Eisen/Gold und Eisen/Silber auftritt. Bereiche kohärenter Spannung I und inkohärenter Grenzfläche II. 
renten Bereichen an Korngrenzen senkrecht zur Schicht (II, Abbildung 7.1) wird demnach ein großer Gradient der Grenzflächenenergie von bis zu einigen $5 \cdot 10^{8} \mathrm{~J} / \mathrm{m}^{3}$ $\left(10 \mathrm{~J} / \mathrm{m}^{2}\right.$ auf etwa $\left.20 \mathrm{~nm}\right)$ vorliegen. Da die Grenzflächenkrümmung im Vergleich dazu nur einen schwachen Gradienten von etwa $10^{7} \mathrm{~J} / \mathrm{m}^{3}$ (Wellenlänge $40 \mathrm{~nm}$ ) erzeugt, ist der Gradient in der Spannungsenergie wohl dominierend. Bei inkohärenten Grenzflächen ist der strukturelle Beitrag im Bereich I und Bereich II in etwa gleich, d.h. es gibt keinen Gradienten aufgrund von unterschiedlichen Spannungen. Liegen kohärente Grenzflächen mit geringer Fehlpassung vor, sind die Spannungen entsprechend klein. Dieser Beitrag muss, um eine Diffusion antreiben zu können, jedoch größer sein als der strukturelle Beitrag der Korngrenze der Makrokörner. Ist dies nicht der Fall, liegt kein Gradient der Spannung zwischen den Bereichen I und II (Abbildung 7.1) vor oder es ist im Extremfall sogar denkbar, dass der strukturelle Beitrag der Korngrenze den der kohärenten Grenzfläche überwiegt und ein umgekehrter Gradient von der Korngrenze ins Innere der Makrokörner vorliegt. Ein solcher Fall wurde jedoch nie beobachtet. In den zuletzt genannten Fällen kann die Variation der Krümmung der Grenzfläche aufgrund der geringen Spannungsgradienten als treibende Kraft für eine Diffusion in der Schichtebene angesehen werden.

Ein Schlüsselexperiment zur Klärung der Frage, wann welcher Mechanismus vorliegt, ist die Messung der Desintegrationstemperatur in Abhängigkeit von der Dicke der interkalierten Lage. Es zeigte sich, dass in den Systemen Niob/Kupfer und Eisen/Gold für dünne interkalierte Schichten eine reziproke Abhängigkeit dieser Größen besteht. Ab einer kritischen Dicke steigt die Desintegrationstemperatur dagegen sehr viel stärker an. In Kapitel 5.5 wurde bereits dargestellt, dass die Energie eines kohärenten Schichtsystems aufgrund der Spannungen an den Grenzflächen ebenfalls eine reziproke Abhängigkeit von der Periodenlänge des Schichtpaketes zeigt. Es kann daher angenommen werden, dass durch die Vergrößerung der Periodenlänge die Energiedifferenz zwischen kohärenten Bereichen (I) und inkohärenten Bereichen (II) verkleinert wird, wodurch die treibende Kraft um den gleichen Wert abnimmt. Das bedeutet zum einen, dass in dem Bereich der reziproken Abhängigkeit die Variation der Grenzflächenenergie aufgrund von Spannungen die primäre treibende Kraft der Desintegration ist. Es bedeutet aber auch, dass nicht nur im System Eisen/Gold und Eisen/Silber kohärente Grenzflächen vorliegen, sondern 
auch im System Niob/Kupfer für Nioblagendicken unter 1,5 nm ein partiell kohärentes Wachstum der Lagen auftreten sollte. Solche Stellen lokalen epitaktischen Wachstums von Kupfer auf Niob werden im TEM tatsächlich beobachtet (Abbildung 7.5, Seite 82). Überschreitet die Lagendicke der interkalierten Schicht den kritischen Wert, können Spannungen durch Bildung von semi- oder inkohärenten Grenzflächen abgebaut werden und die Krümmung der Grenzflächen wird für die Stabilität der Grenzflächen entscheidend. Ein Beweis für diese Theorie liefern direkt durchstrahlbare Dreifachschichten vom Typ Gold/Eisen/Gold auf KohlenstoffNetzchen. Aufgrund der großen Oberflächenspannung zwischen Gold und Kohlenstoff wächst die erste Goldlage mit einer geringen Korngröße auf. Für geringe Eisenlagendicken $(<2,5 \mathrm{~nm})$ wachsen die Eisenlagen und die nächste Goldlage epitaktisch, die Korngröße des Goldes bleibt so klein wie in der ersten Lage. Ist die Eisenlage dicker, so wächst die nächste Goldlage nicht mehr kohärent und die Korngröße wird durch die Grenzflächenspannung zwischen Eisen und Gold bestimmt. Dadurch ist die Gold-Korngröße in diesem Fall in der zweiten Lage deutlich größer.

Eine weitere Information, die Ausgangsform:

man aus den Röntgendiffraktogrammen gewinnt, ist, dass die Relaxation der Metalle bei Schichtpaketen, die aufgrund von Spannungsgradienten zerfallen, sehr schnell nach Beginn der Desintegration abgeschlossen ist. Gleichzeitig haben sich die Nanopartikel gebildet. Diese wachsen jedoch auch nach der Relaxation der Grenzflächen weiter, das heißt, dass nach dem Abbau der Spannungen der weitere Fortgang der Desintegration durch die Krümmungen der Grenzflächen bestimmt

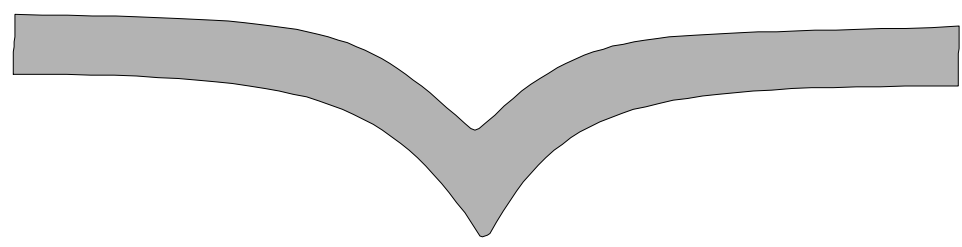

1. Schritt: Spannungsabbau

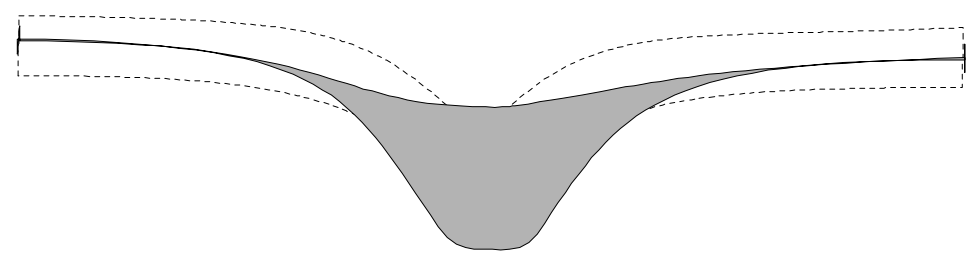

2. Schritt: Krümmungsabbau

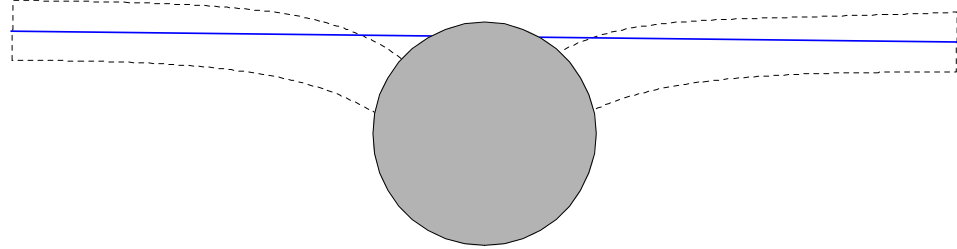

Abbildung 7.2: Schritte der Veränderung der Mikrostruktur. 
wird. Wie ein solcher Prozess ablaufen könnte, zeigt die Abbildung 7.2 schematisch. Da die Spannungsgradienten an Ober- und Unterseite der Schicht als gleich angenommen werden können, sollte sich die Form der Grenzfläche bei der Reduktion der Spannungen nur dort verändern, wo das Partikel gebildet wird. Nachdem die Schicht zerfallen ist, weisen die „Risskanten“ extrem große Krümmungen auf. Zum Abbau der Variation der Krümmungen der Grenzfläche findet ein zweiter Schritt statt, bei dem zunächst schnell die Spitzen der Partikel innerhalb der Grenzflächen abstumpfen und der dann zur Abrundung des Partikels und zur Glättung der Grenzfläche führt. Während dieses Prozesses bilden sich glatte Grenzflächen und Partikel, die gegenüber den neuen Grenzflächen nach unten versetzt sind.

Im Fall geringer Spannungsvariationen findet der erste Schritt nicht statt und die Diffusion wird von Beginn an durch die Variation der Krümmung bestimmt. Die Veränderung der Mikrostruktur, die dabei zu erwarten ist, wurde bereits in Kapitel 5.4 simuliert. Dabei zeigte sich, dass neben den Partikeln, die sich in den Makrokorngrenzen bilden, auch diskusförmige Partikel innerhalb der Makrokörner entstehen. Diese werden dadurch verursacht, dass es innerhalb der Makrokörner Bereiche mit fast verschwindender Krümmung gibt. Im Fall einer spannungsgetriebenen Desintegration können solche Partikel nicht entstehen, da große Spannungen, wie in Kapitel 3.2 dargelegt wurde, zu einer geringen Makrokornbreite bzw. einer großen Krümmung der gesamten Grenzfläche führen.

Beide Mechanismen führen dazu, dass die Partikelbildung bevorzugt an den Makrokorngrenzen stattfindet. Die Partikel sind dabei gegenüber der neuen Grenzfläche senkrecht verschoben. Diese Verschiebung wird aus der plausibel und wird in dieser Form auch im TEM

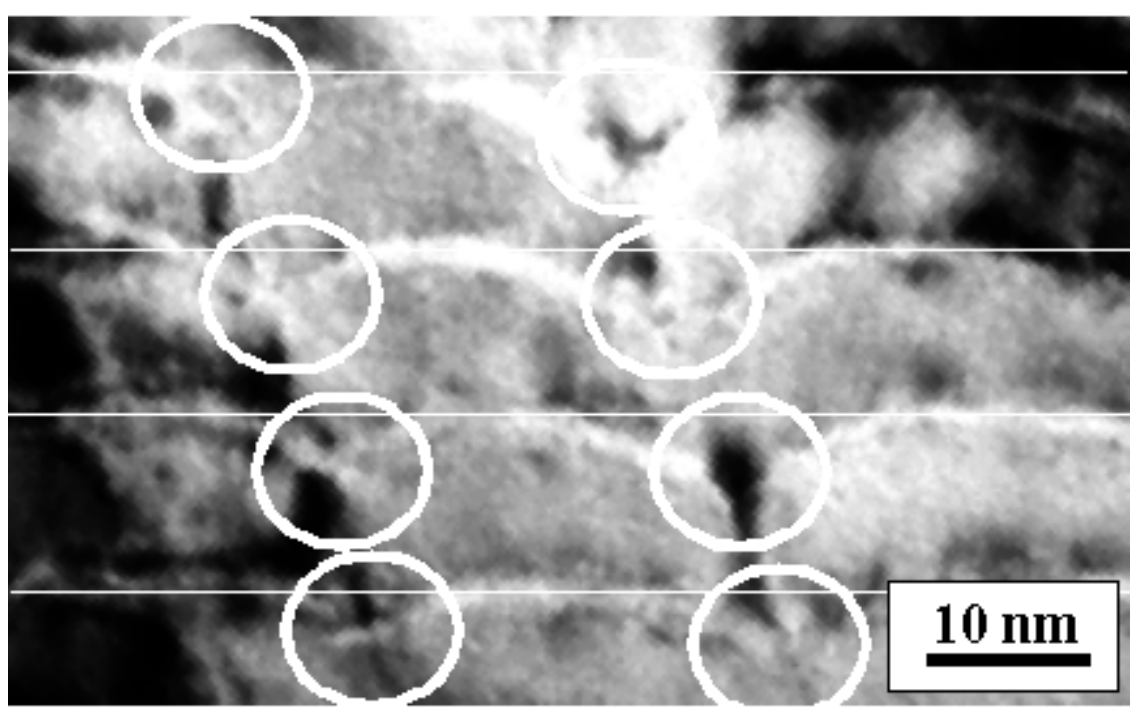

Abbildung 7.3: Unbehandelte Eisen/Gold-Vielfachschicht. Die blauen Linien kennzeichnen die Position der späteren Grenzflächen und die grünen Kreise die möglichen Position für Partikel. 
beobachtet (Abbildung 7.3, Abbildung 7.4).

Diese Überlegungen lassen sich auf die Systeme Eisen/Gold und Eisen/Silber anwenden, die trotz vergleichbarer Fehlpassung deutliche Unterschiede in den Desintegrationstemperaturen zeigen. In unbehandelten Eisen/Silber-Schichtpaketen sind die Breiten der Makrokörner relativ groß $(80-90 \mathrm{~nm})$ und die

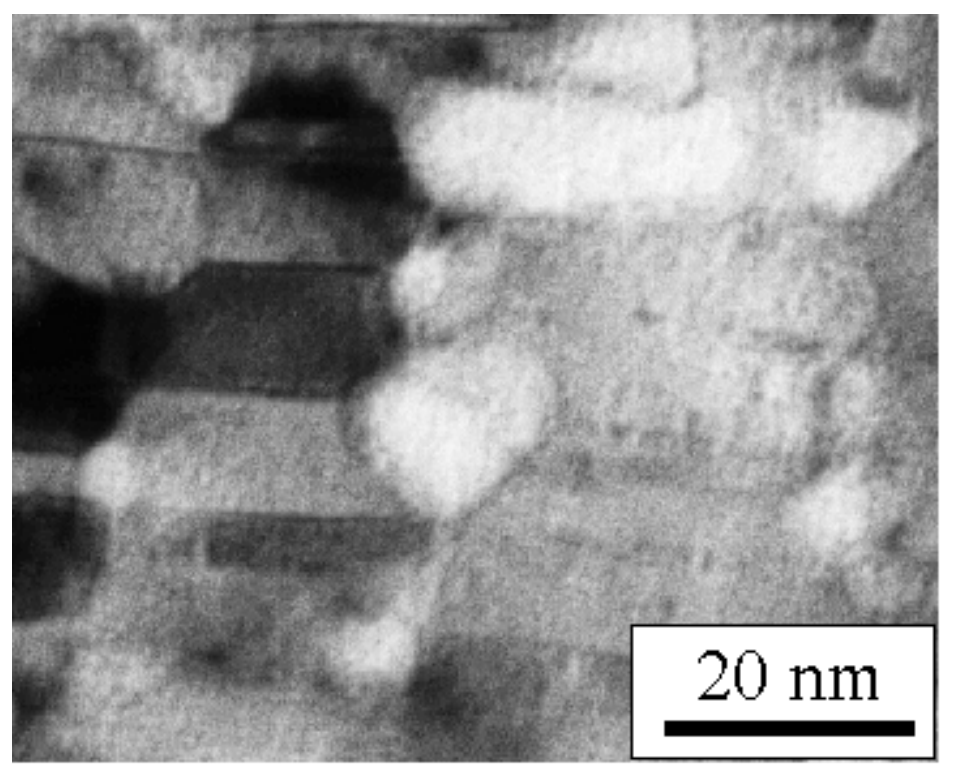

Abbildung 7.4: Bildung von Eisenpartikeln in den Makrokorngrenzen unterhalb der neuen Grenzflächen. Grenzflächen in der Mitte der

Körner nur schwach gekrümmt. Demgegenüber beträgt die Makrokornbreite in Eisen/Gold-Vielfachschichten nur ca. $30 \mathrm{~nm}$. Die Grenzflächen sind entsprechend überall signifikant gekrümmt. Nach der Desintegration findet man in beiden Systemen glatte Grenzflächen und Partikel, die gegenüber der neuen Grenzfläche verschoben sind. Im Eisen/Silber findet man zusätzlich noch Partikel in der Mitte der Makrokörner.

Die Zwillingsdichte ist in den Silberlagen deutlich größer als in den Goldlagen. Zudem tritt in den Silberlagen eine frühe Erholungsstufe auf, die die Spannungen bei Temperaturen deutlich unterhalb der Desintegrationstemperatur noch weiter verringert. Daher ist ein Großteil der Spannungen in diesem System bereits vor Beginn der Desintegration abgebaut. Im Wesentlichen wird die Desintegration daher von der Variation der Grenzflächenkrümmung bestimmt. Spannungen haben nur zu Beginn einen unterstützenden Einfluss, solange bis die Restspannungen abgebaut sind. Demgegenüber findet im System Eisen/Gold die Desintegration spannungsgetrieben bei niedrigen Temperaturen statt. Überschreitet die Eisenlagendicke den kritischen Wert, können die Spannungen im Schichtsystem relaxieren und die treibende Kraft wird nun durch die Variation der Krümmung bestimmt. Die Reduktion der treibenden Kraft, die dieser Übergang mit sich bringt, ist im Anstieg der Desintegrationstemperatur mit der Lagendicke deutlich zu erkennen. Im System Eisen/Gold wird die Desintegration also im Falle kohärenter Grenzflächen primär durch Variationen 
der Spannung und im Falle inkohärenter Grenzflächen primär durch Variationen der Grenzflächenkrümmung getrieben.

Auch im System Niob/Kupfer ist der Übergang vom spannungs- zum krümmungsgetriebenen Zerfall nachzuvollziehen. Nur in sehr dünnen Nioblagen mit weniger als $1,5 \mathrm{~nm}$ Dicke ist ein kohärentes stark verspanntes Wachstum zu beobachten. Bei der isothermen Messung der Niobpartikelgröße in Abhängigkeit von der Zeit zeigt sich daher ein sehr schneller Zerfall, gefolgt von einem deutlichen Wachstum der Niobpartikel (Abbildung 4.3, Seite 39). Bei den beobachteten Partikelgrößen findet aufgrund der nur $4 \mathrm{~nm}$ dicken

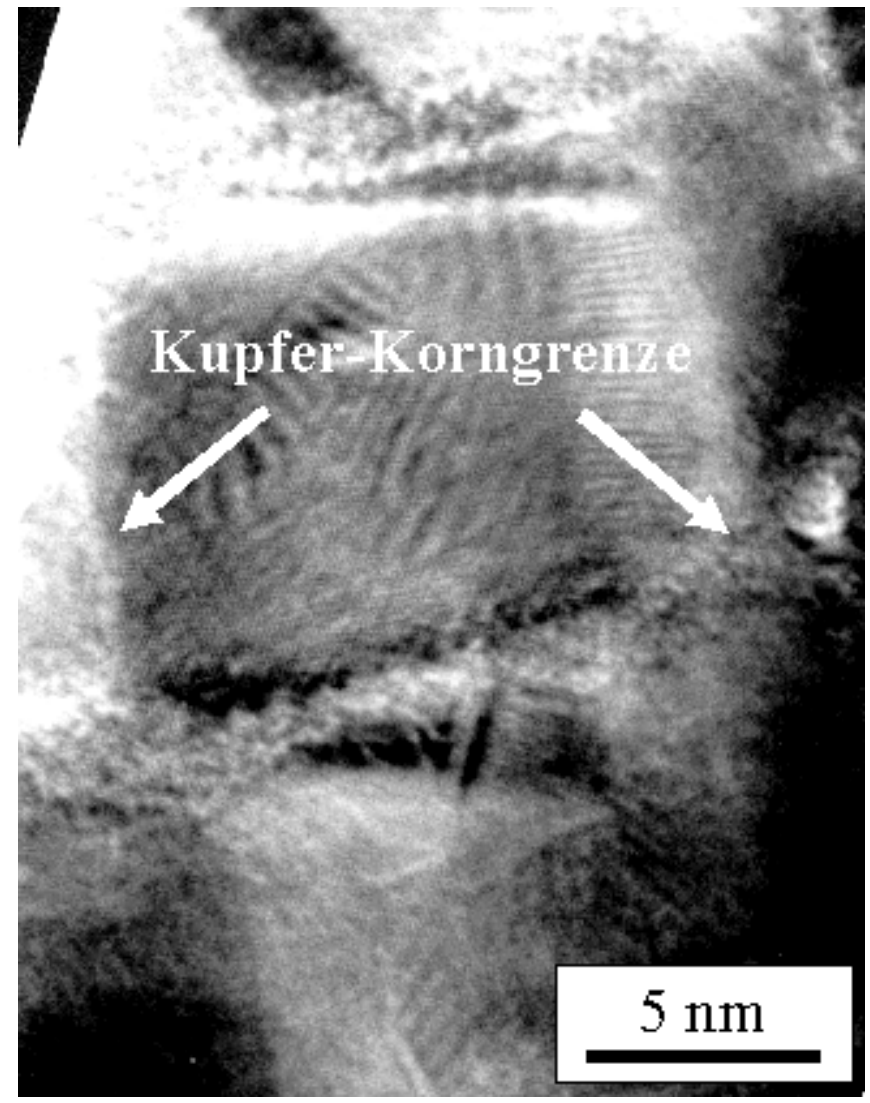

Abbildung 7.5: TEM-Bild von einer Niob/KupferVielfachschicht. Es ist eine konstante Orientierung der Kupferkörner durch Nioblage hindurch zu erkennen. Kupferschicht eine Agglomeration der Partikel statt. Der Abstand, in dem sich die Partikel beim spannungsgetriebenen Zerfall bilden, wird auch hier durch die Breite der kohärenten Bereiche bestimmt und liegt in diesem System, wie in Abbildung 7.5 zu erkennen ist, bei etwa $10 \mathrm{~nm}$. Geordnete Strukturen sind im System Niob/Kupfer nicht zu erwarten, da die Spannungen zu groß sind, um über viele Lagen kohärente Strukturen aufbauen zu können. Makrokörner werden daher nicht gefunden. Beim Überschreiten der kritischen Lagendicke, erhöht sich auch im System Niob/Kupfer die Desintegrationstemperatur deutlich, was den Übergang vom spannungs- zum krümmungsgetriebenen Zerfall anzeigt. Die Niob/Kupfer-Schichten weisen eine kurzwellige Rauigkeit, die mit der Korngröße des Kupfers $(\sim 10 \mathrm{~nm})$ korreliert ist, aber auch eine deutlich langwelligere Modulation der Schichtstruktur auf (vgl. Kapitel 3.1). Beim krümmungsgetriebenen Zerfall scheint die kurzwellige Rauigkeit keine Rolle zu spielen, da die TEM bzw. XRM-Bilder zeigen, dass die Partikelgröße und der Partikelabstand deutlich größer als $10 \mathrm{~nm}$ sind. Die geringen von der 
langwelligen Rauigkeit induzierten treibenden Kräfte zeigen sich auch bei der Kinetik der Partikelbildung. Selbst bei einer relativ hohen Auslagerungstemperatur von $600^{\circ} \mathrm{C}$ ist das Einsetzen der Desintegration erst nach einer Stunde Auslagerung zu beobachten (vgl. Abbildung 4.3). Im System Niob/Kupfer wird die Desintegration also für sehr dünne Nioblagen durch Variationen der Spannung und für dickere Lagendicken durch Variationen der Grenzflächenkrümmung getrieben.

Systeme die glatte und unverspannte Grenzflächen haben, sollten nach den gefundenen Ergebnissen keine Gestaltinstabilität zeigen. Das hat sich an den Systemen Kobalt/Kupfer und Kobalt/Kohlenstoff auch bestätigt. In diesen Systemen ist eine Gestaltinstabilität nur durch Korngrenzkriechen oder Rekristallisation zu erwarten. Diese Prozesse unterscheiden sich von der hier vorgestellten Gestaltinstabilität dadurch, dass sie in der Regel erst bei Temperaturen deutlich oberhalb der hier gefundenen $600^{\circ} \mathrm{C}$ auftreten. Im System Kobalt/Kohlenstoff ist eine grenzflächengetriebene Desintegration allerdings möglich, wenn der amorphe Kohlenstoff kristallisiert. Durch hinreichend dünne Kohlenstofflagen kann die Kristallisationstemperatur aber zu sehr hohen Werten verschoben werden.

\subsection{Weitere Einflussfaktoren der Desintegration}

Die Ergebnisse des letzten Kapitels haben gezeigt, dass eine Vielzahl von Aspekten der thermischen Zersetzung von Schichtpaketen nur durch detailierte Kenntnis der Mikrostruktur zu verstehen sind. Es stellt sich nun die Frage, ob ein zumindest qualitatives Verständnis des Zerfallsprozesses durch charakteristische Materialparameter möglich ist. In diesem Zusammenhang soll auch auf den bisher nicht weiter betrachteten Aspekt der Kinetik der Desintegration eingegangen werden.

Setzt man ein Schichtpaket mit kohärenten Grenzflächen voraus, so dass keine bevorzugte Diffusion in der eigentlichen Grenzfläche stattfindet, erfolgt die Partikelbildung durch eine Volumendiffusion parallel zu den Grenzflächen. Die entsprechenden Diffusionskoeffizienten sind aber nicht allein durch thermisch aktivierte Sprungprozesse bestimmt. Die zusätzlichen treibenden Kräfte durch Spannungsgradienten und Grenzflächenkrümmung führen zu einer deutlich erhöhten Beweglichkeit. Mit Hilfe der Ergebnisse der vorherigen Kapitel lassen sich aber die effek- 
tiven Werte für diese relevanten Diffusionskoeffizienten abschätzen. Die Desintegration erfolgt unter Erhaltung des Lagenvolumens, d.h. es findet kein Materialtransport zwischen den Lagen statt (vgl. Kapitel 4.1.3). Auf der charakteristischen Zeitskala der Partikelbildung muss also die im wesentlichen nur thermisch bedingte Diffusion senkrecht zur Lagenstruktur so eingeschränkt sein, dass bestenfalls ein Materialtranport über die Makrokorngrenzen erfolgen kann. Andererseits muss die Diffusion parallel zu den Grenzflächen so groß sein, dass im gleichen Zeitrahmen der durch die Mikrostruktur vorgegebene Diffusionsweg zurückgelegt werden kann.

Bei der Desintegrationstemperatur erfolgt für Niob/Kupfer, Eisen/Gold und Eisen/Silber ein deutliches Partikelwachstum innerhalb von 5 Minuten. Die charakteristische Länge die die Atome des interkalierten Metalls zur spannungsgetriebenen Partikelbildung zurückzulegen haben, ist für Niob/Kupfer die halbe Kupferkorngröße $(\sim 5 \mathrm{~nm})$, für Eisen/Gold die halbe Makrokorngröße $(\sim 10-15 \mathrm{~nm})$ und für Eisen/Silber aufgrund der Partikelbildung in den Makrokörnern ein Drittel der Makrokornbreite $(\sim 30 \mathrm{~nm})$. In Tabelle 7.1 sind die relevanten thermischen Diffusionskoeffizienten zusammengetragen. Eine signifikante Beweglichkeit liegt also nur

\begin{tabular}{||c|c|c|c|c||}
\hline \hline System & $\begin{array}{c}\text { Aktivierungsenergie } \mathbf{Q} \\
{[\mathbf{k c a l} / \mathbf{M o l}]}\end{array}$ & $\begin{array}{c}\boldsymbol{D}_{\mathbf{0}} \\
{\left[\mathbf{c m}^{2} / \mathbf{s e c}\right]}\end{array}$ & $\begin{array}{c}\boldsymbol{D} \text { @ 700K } \\
{\left[\mathbf{c m}^{2} / \mathbf{s e c}\right]}\end{array}$ & $\begin{array}{c}\boldsymbol{D} @ \mathbf{8 0 0 K} \\
{\left[\mathbf{c m}^{2} / \mathbf{s e c}\right]}\end{array}$ \\
\hline Eisen in Gold & 41,6 & 0,08 & $9,96 \mathrm{E}-015$ & $4,09 \mathrm{E}-013$ \\
\hline Gold in Gold & 40,2 & 0,07 & $2,37 \mathrm{E}-014$ & $8,58 \mathrm{E}-013$ \\
\hline Eisen in Eisen & 60,3 & 5,4 & $1,06 \mathrm{E}-018$ & $2,32 \mathrm{E}-016$ \\
\hline Gold in Eisen & 62,4 & 31 & $1,36 \mathrm{E}-018$ & $3,58 \mathrm{E}-016$ \\
\hline Eisen in Silber & 49,04 & 2,42 & $1,48 \mathrm{E}-015$ & $1,18 \mathrm{E}-013$ \\
\hline Silber in Silber & 45,2 & 0,67 & $6,38 \mathrm{E}-015$ & $3,61 \mathrm{E}-013$ \\
\hline Eisen in Eisen & 60,3 & 5,4 & $1,06 \mathrm{E}-018$ & $2,32 \mathrm{E}-016$ \\
\hline Silber in Eisen & 69 & 1950 & $7,68 \mathrm{E}-019$ & $3,64 \mathrm{E}-016$ \\
\hline Niob in Kupfer & 60,1 & 2,04 & $4,63 \mathrm{E}-019$ & $9,92 \mathrm{E}-017$ \\
\hline Kupfer in Kupfer & 50,5 & 0,78 & $1,68 \mathrm{E}-016$ & $1,53 \mathrm{E}-014$ \\
\hline Niob in Niob & 96 & 1,1 & $1,82 \mathrm{E}-030$ & $9,63 \mathrm{E}-027$ \\
\hline \hline
\end{tabular}

Tabelle 7.1: Tabelle der Tracer-Diffusionskoeffizienten von Eisen, Silber und Gold. 


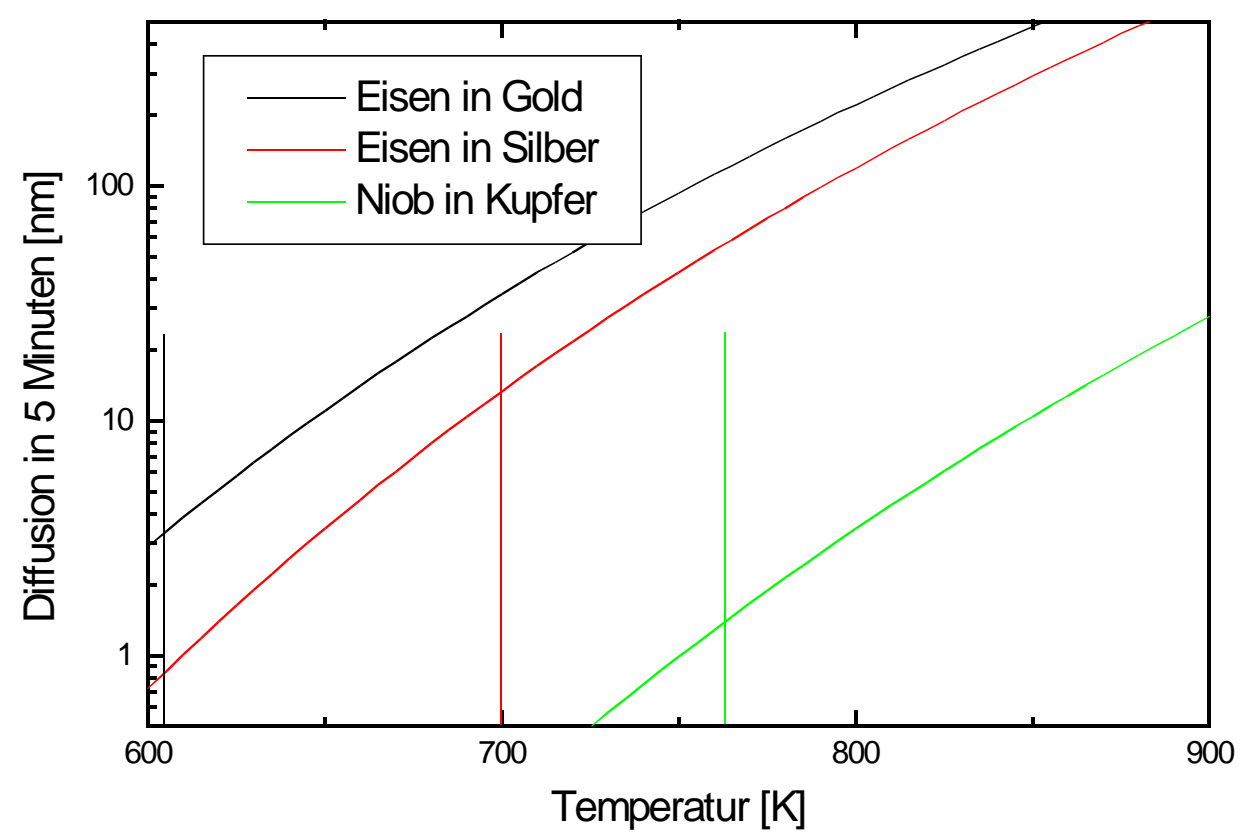

Abbildung 7.6: Diffusionskonstanten D berechnet in Abhängigkeit von der Temperatur. Dargestellt ist die Diffusion von Eisen in Gold (durchgehend), Eisen in Silber (gestrichelt) und Niob in Kupfer (gestrich-punktet). Die senkrechten Striche markieren die Desintegrationstemperaturen für die verschiedenen Schichtsysteme bei einer Zusammensetzung von $2 \mathrm{~nm}$ zu $10 \mathrm{~nm}$.

für die Selbstdiffusion und für die Diffusion des interkalierten Metalls im Matrixmetall vor. Die relativ schnelle Selbstdiffusion spiegelt sich auch in der Glättung der Grenzflächen der Edelmetalle wieder, nachdem die interkalierte Komponente herausdiffundiert ist. Trägt man nun die mittleren Diffusionswege (gemäß $x=2 \sqrt{D t}$ ), die in 5 Minuten erreicht werden, gegen die Temperatur auf (Abbildung 7.6), erhält man einen guten Maßstab für die Desintegrationstemperatur der verschiedenen Systeme. Um eine bessere Vergleichbarkeit der Kurven mit den Messergebnissen zu haben, wurden in die Auftragung zusätzlich die Desintegrationstemperaturen für Schichtpakete mit $2 \mathrm{~nm}$ interkalierter Lagendicke und $10 \mathrm{~nm}$ Matrixlagendicke für Eisen/Silber und Eisen/Gold und mit 1,5 nm interkalierter Lagendicke und $10 \mathrm{~nm}$ Matrixlagendicke für Niob/Kupfer eingetragen. Im letzten Fall wurde die Nioblagendicke etwas dünner gewählt, um in allen Systemen einen spannungsgetriebenen Zerfall vergleichen zu können. Außerdem kann nur im Fall von kohärenten Grenzflächen die verstärkte Diffusion entlang von inkohärenten Grenzflächen unberücksichtigt bleiben. 
Bei der Desintegrationstemperatur kann Niob gut $1 \mathrm{~nm}$ in Kupfer, Eisen in Silber etwa $10 \mathrm{~nm}$ und Eisen in Gold etwa $4 \mathrm{~nm}$ diffundieren. Nur beim System Eisen/Silber wäre also überhaupt ein Materialtranport zwischen den Lagen möglich.

Vergleicht man nun diese zurückgelegten Wege mit den charakteristischen Längen der Diffusion parallel zu den Grenzflächen, so zeigt sich für alle Systeme, dass der Diffusionskoeffizient etwa einen Faktor 6 größer ist. Somit ergibt sich folgendes Bild. Da die Selbstdiffusion der interkalierten Komponenten sehr klein ist, ist sie wahrscheinlich auch im spannungsgetriebenen Fall vernachlässigbar, die Diffusion erfolgt über das Matrixmetall. Schon aus diesem Gesichtspunkt folgt, dass eine thermische Diffusion senkrecht zur Schicht notwendig ist, um die Desintegration auszulösen. Die Desintegrationstemperatur steigt also mit abnehmendem Diffusionskoeffizienten des interkalierten Metalls in der Matrix. Speziell für die hier betrachteten Systeme gilt, dass bei der Zerfallstemperatur in der charakteristischen Zerfallszeit etwa die Hälfte bis ein Drittel der charakteristischen Länge durch thermische Diffusion zurückgelegt werden kann.

Es wurde schon in Kapitel 5.5 darauf hingewiesen, dass bei gegebener Fehlpassung die elastische Energiedichte in der härteren Komponente größer ist als in der weicheren. Man würde daher erwarten, dass bei spannungsgetriebener Desintegration die Desintegrationstemperatur mit dem zunehmenden Unterschied der elastischen Module von interkalierter Schicht und Matrix abnimmt. Im Modell von Sridhar [Sridhar et al., 1997b] (vgl. Kapitel 5.5) wird dieser Sachverhalt durch den

\begin{tabular}{|l|c|c|}
\hline Metall & $\begin{array}{c}\text { Elastizitätsmodul [GPa] } \\
\text { [Smithells] }\end{array}$ & $\alpha=\frac{\left(E_{A}-E_{B}\right)}{\left(E_{A}+E_{B}\right)}$ \\
\hline Eisen & 211,4 & $\alpha_{\mathrm{Fe} / \mathrm{Au}}=0,458$ \\
\hline Gold & 78,5 & $\alpha_{\mathrm{Fe} / \mathrm{Ag}}=0,438$ \\
\hline Kobalt & 211 & $\alpha_{\mathrm{Nb} / \mathrm{Cu}}=0,105$ \\
\hline Kupfer & 129,8 & $\alpha_{\mathrm{Co} / \mathrm{Cu}}=0,238$ \\
\hline Niob & 170,3 & \\
\hline Silber & 82,7 & \\
\hline
\end{tabular}

Tabelle 7.2: Elastizitätsmoduln und Faktor für die verschiedenen Metalle und Metallsysteme.
Parameter $\alpha$ beschrieben, der positiv sein muss, um eine Desintegration auszulösen. In der Tabelle 7.2 sind die Werte für $\alpha$ für die unterschiedlichen Metallkombinationen angegeben. Tendenziell sind tatsächlich die Schichtpakete mit großem $\alpha$ durch eine niedrige Zerfallstemperatur gekennzeichnet. Nach den Ergebnissen des vorigen Kapitels ist 
aber nur für die Systeme Eisen/Gold bzw. Niob/Kupfer ein spannungsgetriebener Mechanismus der Desintegration zu erwarten. Zumindest für diese beiden Systeme ist ein großer Unterschied in $\alpha$ mit großen Unterschieden in den Zerfallstemperaturen verbunden.

Nach dem Modell von Sridhar [Sridhar et al., 1997a] wäre streng genommen zu erwarten, dass eine weiche interkalierte Schicht in einer harten Matrix nie spannungsgetrieben desintegriert. Experimentell sichergestellt ist, dass der Übergang von einem Schichtpaket der Zusammensetzung Eisen/Gold $=2 \mathrm{~nm} / 10 \mathrm{~nm} \quad \mathrm{zu}$ Eisen/Gold $=10 \mathrm{~nm} / 2 \mathrm{~nm}$ die Desintegrationstemperatur von $320^{\circ} \mathrm{C}$ auf über $420^{\circ} \mathrm{C}$ vergrößert. Bei dieser Temperatur zeigte das Schichtpaket im TEM noch keine Anzeichen für eine Desintegration (Abbildung 7.7). Durch den entsprechenden Übergang von Niob/Kupfer $=2 \mathrm{~nm} / 10 \mathrm{~nm}$ zu Niob/Kupfer $=10 \mathrm{~nm} / 2 \mathrm{~nm}$ steigt die Desintegrationstemperatur von $475^{\circ} \mathrm{C}$ auf $580^{\circ} \mathrm{C}$. Es kann aus den vorliegenden Daten nicht geschlossen werden, dass bei diesen Experimenten ein Übergang von einem spannungsgetriebenen zu einem krümmungsgetriebenen Mechanismus auftritt. Bemerkenswert ist zumindest, dass die Vergrößerung der Desintegrationstemperatur für das System mit großem $\alpha$ (Eisen/Gold) deutlich stärker ausfällt als für Niob/Kupfer. Falls die Spannungen noch eine Rolle spielen, dann erhöht sich die Stabilität eines Schichtpakets deutlich, wenn man statt der harten Komponente die weichere interkaliert.

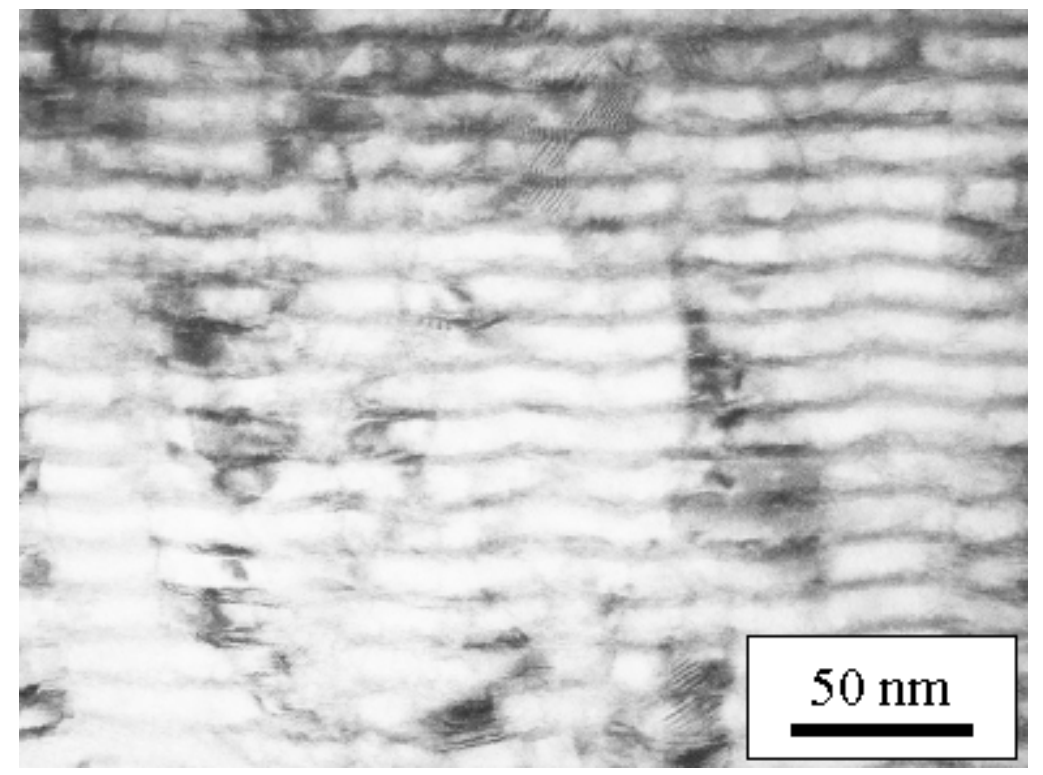

Abbildung 7.7:TEM-Bild einer 60 Minuten bei $420^{\circ} \mathrm{C}$ ausgelagerten Eisen/Gold-(10 $\mathrm{nm} / 2 \mathrm{~nm})$-Vielfachschicht. 


\section{Zusammenfassung}

Im Rahmen dieser Dissertation wurde die thermisch aktivierte Gestaltinstabilität von Vielfachschichten aus nicht mischenden Metallen untersucht. Dazu wurden Niob/Kupfer, Eisen/Gold, Eisen/Silber, Kobalt/Kupfer und Kobalt/Kohlenstoff Vielfachschichten deponiert und mittels Röntgenbeugung, Röntgenmikroskopie und Transmissionselektronenmikroskopie vor, während und nach der Auslagerung charakterisiert.

Es zeigte sich, dass die Systeme Eisen/Gold und Eisen/Silber trotz großer Fehlpassung kohärente Grenzflächen basierend auf der Nishiyama-Wassermann-Beziehung aufbauen. Die Orientierung der Edelmetallkörner wurde dabei durch die dünneren Eisenlagen hindurch von Lage zu Lage weitergegeben, so dass sich Säulen gleicher kristallographischer Orientierung ausbildeten, die im Falle von Eisen/Gold eine Breite von etwa $30 \mathrm{~nm}$ und im Eisen/Silber eine Breite von 80-90 nm und eine Höhe von mehreren $100 \mathrm{~nm}$ aufwiesen. Die Grenzflächen innerhalb dieser Makrokörner sind gebogen, um die starken Spannungen an der kohärenten Grenzfläche zu reduzieren. Im System Niob/Kupfer zeigte sich ein solchermaßen korreliertes Wachstum nicht, Kupferkörner gleicher Orientierung mit typischen Breiten von $10 \mathrm{~nm}$ geben ihre Orientierung höchstens über 3 Perioden hinweg weiter. Hier treten zwei Sorten von Rauigkeiten auf, eine kurzwellige erzeugt durch die Kornstruktur des Kupfer und eine langwellige Wachstumsrauigkeit. Das System Kobalt/Kupfer wächst zunächst nicht sehr glatt auf, glättet seine Grenzflächen jedoch während der Auslagerung und reduziert seine Grenzflächenenergie durch eine Rekristallisation des Kobalt und Kupfer von (111)-orientierten Körnern zu (100)-orientierten Körnern an den Grenzflächen. Das System Kobalt/Kohlenstoff zeigt ein Lagenwachstum und weist daher sehr glatte Grenzflächen auf.

Röntgenbeugungsuntersuchungen offenbaren, dass in den Systemen Niob/Kupfer und Eisen/Gold nur unterhalb einer kritischen Lagendicke von 1,5 nm bzw. 2,5 nm ein kohärentes Wachstum stattfindet. Dies spiegelt sich in einer reziproken Abhängigkeit der Desintegrationstemperatur von der Lagendicke der interkalierten Lage wieder, die für kohärente Schichtsysteme erwartet wird. In diesem Fall wird die Gestaltinstabilität der Schichtpakete durch eine Variation der Spannungsenergie zwischen den kohärenten Grenzflächen und den inkohärenten Bereichen an den 
Makrokorngrenzen induziert. Konnten die Spannungen vor Einsetzen der Desintegration durch Erholung relaxieren, wie es im System Eisen/Silber beobachtet wurde, oder ist die Lagendicke der interkalierten Schicht so groß, dass sich semi- oder inkohärente Grenzflächen gebildet haben, ist die lokale Variation der Grenzflächenkrümmung die primär treibende Kraft. Mit diesen beiden Modellen lässt sich erklären, wann sich Partikel nach der Desintegration auch innerhalb der Edelmetalllagen bilden und dass die Grenzflächen nach der Desintegration glatt sind. Im Fall der Systeme, die bei der Deposition Makrokörner bilden, wird eine teilweise geordnete Struktur gebildet, da die Eisenpartikel zentral innerhalb der Makrokörner oder aber in Perlenketten senkrecht zur Schicht in den Tripelgrenzen der Makrokörner gebildet werden.

Die Desintegrationstemperatur wird maßgeblich von der Mobilität des interkalierten Metalls im Matrixmetall bestimmt. Im Falle des spannungsgetriebenen Zerfalls wurde gefunden, dass die thermodynamische Diffusion mindestens ein Fünftel des Weges ermöglichen muss, den die Atome von ihrem ursprünglichen Ort zum Ort des Partikels zurücklegen müssen. Die Desintegrationstemperatur nimmt tendenziell ab, wenn die Matrix im Vergleich zur interkalierten Komponente elastisch weicher ist. Ist die Desintegration nur durch die Variation der Krümmung getrieben, muss der thermodynamische Diffusionsweg um etwa das 2 bis 3 fache größer sein.

Die Systeme Kobalt/Kupfer und Kobalt/Kohlenstoff weisen keine Gestaltinstabilität aufgrund der Lagenstruktur auf. Kobalt/Kupfer-Vielfachschichten zeigen bei Temperaturen oberhalb von $700^{\circ} \mathrm{C}$ eine Zerstörung der Lagenstruktur aufgrund von Rekristallisation, die sich von der untersuchten Gestaltinstabilität aufgrund von Grenzflächeneffekten grundsätzlich unterscheidet. In Kobalt/Kohlenstoff ist eine grenzflächengetriebene Desintegration nur durch Kristallisation des amorphen Kohlenstoff möglich. Durch hinreichend dünne Kohlenstofflagen kann die Kristallisationstemperatur aber zu sehr hohen Werten verschoben werden.

Zur Herstellung thermisch stabiler Systeme ist es erforderlich Metalle zu wählen, die geringe mechanische Spannungen aufgrund von Gitterfehlpassung an den Grenzflächen haben und glatte Grenzflächen bilden. Besteht das Ziel darin, geordnete Nanostrukturen zu erzeugen, sind Systeme mit Gitterfehlpassungen zu wählen, die groß genug sind, um die Mikrostruktur zu beeinflussen (wie in den Systemen 
Eisen/Gold und Eisen/Silber), aber nicht zu groß sind, dass eine unregelmäßige Mikrostruktur entsteht (wie im System Niob/Kupfer). Die treibenden Kräfte für eine ordnende Desintegration sind aufgrund der Spannungen und Krümmungen der Grenzfläche in solchen Systemen vorhanden. 


\section{Literaturverzeichnis}

Ariosa et al., 1988:

D. Ariosa, O. Fischer, M.G. Karkut, J.-M. Triscone

Elastic model for the partially coherent growth of metallic superlattices

Physical Review B 37 (1988) 2421-2425

\section{Becker, 1938:}

R. Becker

Annalen der Physik 32 (1938) 128

Bilaniuk et al., 1998:

M. Bilaniuk, J. Howe

Interface Science 6 (1998) 317

Bobeth et al., 1997:

M. Bobeth, R. Krawietz, H. Mai, W. Pompe, A. Sewing, J. Thomas

Morphological transition in Ni/C nanoscale multilayers

Solid State Ionics (1997) 279-284

Borchers, Troche et al., 2000:

C. Borchers, P. Troche, C. Herweg, J. Hoffmann

Microstructure of Thin Magnetic Iron/Gold Multilayer Films

Acta Metallurgica and Materialia (2000)

\section{Borchers et al., 1999:}

C. Borchers, P. Ricardo, C. Michaelsen

Interfacial Wetting and Percolation Threshold in Ultrathin Ni/C Films

Philosophical Magazine A (2000) 1669-1679

\section{Borchers et al., 2000:}

C. Borchers, R. Bormann

Determination of Interface Tension from a Pair Interaction Model

eingereicht bei Philosophical Magazine (2000)

\section{Bormann, 1979:}

R. Bormann

Pulvermetallurgische Herstellung von Nb3Sn- und V3Ga-Faserverbund-SL

Dissertation, Universität Göttingen, 1979

\section{Bruce et al., 1978:}

L.A. Bruce, H. Jäger

Geometric Factors in f.c.c. and b.c.c. Metal-on-Metal epitaxy

Philosophical Magazine A 38 (1978) 223-240 


\section{Cahn et al, 1958:}

J.W. Cahn, J.E. Hilliard

Journal of Chemical Physics 28 (1958) 258

\section{Clemens, 1984:}

B.M. Clemens

Amorphous Zr-Ni Films Formed by Solid State Reaction

Journal of Non-Crystal Solids 61 (1984) 817-821

\section{Clemens, 1997:}

W. Clemens, H.v.d. Berg, G. Rupp, W. Schelter, M. Vieth, J. Wecker

Journal of Applied Physics 81 (1997) 4310

\section{Cline, 1971:}

H.E. Cline

Shape Instabilities of Eutectic Composites at Elevated Temperatures

Acta Metallurgica 19 (1971) 481-490

Cullity, 1967:

B.D. Cullity, Elements of X-Ray Diffraction

Addison-Wesley Publishing Company, 1967

\section{Drüsedau et al., 1999:}

T. P. Drüsedau, T. Bock, Th.-M. John, F. Klabunde, W. Eckstein Energy transfer into the growing film during sputter deposition....

Journal of Vacuum Science and Technology A 17 (1999) 2896-2905

\section{Ewert, 1998:}

J. Ewert

Ionenstrahlungsinduzierte Phasenumwandlungen im System Nickel-Aluminium

Dissertation, Universität Göttingen, 1998

Ewert et al., 1997:

J.C. Ewert, F. Hartung, G. Schmitz

Measurement of Interdiffusion by High Angle Hollow Cone Illumination

Applied Physics Letters 71 (1997) 1311-1313

Frey, 1987:

H. Frey, G. Kienel, Dünnschicht Technologie

VDI-Verlag GmbH, 1987

\section{Haasen K12, 1994:}

P. Haasen, Physikalische Metallkunde

Springer Verlag Heidelberg, 1994

\section{Haasen K13, 1994:}

P. Haasen, Physikalische Metallkunde

Springer Verlag Heidelberg, 1994 


\section{Haasen K3, 1994:}

P. Haasen, Physikalische Metallkunde

Springer Verlag Heidelberg, 1994

\section{Hamp, 1997:}

M. Hamp

Charakterisierung laserdeponierter Schichten u. Schichtsysteme

Diplomarbeit, Universität Göttingen, 1997

\section{Hartung, 1996:}

F. Hartung

Untersuchung der Interreaktion von $\mathrm{Cu} / \mathrm{Au}$ mit Hilfe der Z-Kontrast ELMI

Diplomarbeit, Universität Göttingen, 1996

\section{Hasson et al., 1972:}

G. Gasson, J.-Y. Boos, I. Herbenval, M. Biscondi, C. Goux

Theoretical and Experimental Determination of Grain Boundary Structure

Surface Science 31 (1972) 115-137

\section{Hattenhauer, 1992:}

R. Hattenhauer

BFZA-Elektronenmikroskopie der Entmischung von $\mathrm{Cu} / \mathrm{Co}$

Dissertation, Universität Göttingen, 1992

Hattenhauer et al., 1993:

$R$. Hattenhauer, P. Haasen

The Decomposition Kinetics of Cu- 1 at $\%$ Co at $823 \mathrm{~K}$

Philosophica Magazine A 68 (1993) 1195-1213

\section{Hentschel et al., 1998:}

M. Hentschel, M. Bobeth, G. Diener, W. Pompe

Theoretical Analysis of the Evolution of Composition Profiles in Nano-ML Proceedings of TATF 1998 (1998)

\section{Herweg, 1999:}

C. Herweg

GMR als Sonde zur Untersuchung thermisch induzierter Gestaltinstabilitäten

Diplomarbeit, Universität Göttingen, 1999

\section{Hoffmann, 1991:}

\section{J. Hoffmann}

Präparation und Untersuchung der sl Eigenschaften von NbAl-Dünnfilmen

Diplomarbeit, Universität Göttingen, 1991

\section{Josell, 1999:}

D. Josell, F. Spaepen

Surfaces, Interfaces, and Changing Shapes in Multilayered Films

MRS Bulletin (1999) 39-43 
Kittel, 1993:

Ch. Kittel, Einführung in die Festkörperphysik

R. Oldenburger Verlag GmbH, München, 1993

Konno et al., 1994:

T.J. Konno, R. Sinclair

Crystallization of Amorphous Carbon in Carbon-Cobalt Layered Thin Films

Acta Metallurgica and Materialia 43 (1994) 471-484

Krebs, 1984:

H. $-U$. Krebs

Strukturuntersuchungen an amorphen und kirstallinen (Zr,Hf)-Fe-Legierungen

Dissertation, Universität Göttingen, 1984

Küpper et al., 1994:

T. Küpper, N. Nasbaum

Simulation of particle growth and ostwald ripening via the Cahn-Hilliard eq

Acta Metallurgica and Materialia 42 (1994) 1847-1858

Labat et al., 1999:

S. Labat, P. Gergaud, O. Thomas, B. Gilles, A. Marty

Segregation and strain relaxation in Au/Ni multilayers: An in situ exp.

Applied Physics Letters 75 (1999) 914-916

\section{Landolt-Börnstein:}

Landolt-Börnstein, Diffusion in festen Metallen und Legierungen

Springer Verlag Heidelberg, Berlin, New York,

\section{Loquet, 1989:}

J.-P. Locquet, D. Neerinck, L. Stockman, Y. Bruynseraede, I.K. Schuller

Discrete and Continuous Disorder in Superlattices

Physical Review B 39 (1989) 13338-13342

\section{Lovesey, 1996:}

S.W. Lovesey, S.P. Collins

$\mathrm{X}$-Ray Scattering and Absorption by Magnetic Materials (1996)

\section{Mezey et al., 1982:}

L.Z. Mezey, J. Giber

The Surface Free Energies of Solid Chemical Elements...

Japanese Journal of Applied Physics 21 (1982) 1569-1571

\section{Moffatt, 1984:}

W.G. Moffatt, The Handbook of Binary Phase Diagrams

Genium Publishing Corporation, 1984 
Mullins, 1957:

W.W. Mullins

Theory of Thermal Grooving

Journal of Applied Physics 28 (1957) 333-339

Oelgeschläger, 1984:

D. Oelgeschläger

Phasendiagramm und Bildungskinetik intermetallischer Phasen in CuNiSn

Dissertation, Universität Göttingen, 1984

\section{Okamoto, 1984:}

H. Okamoto, T.B. Massalski, L.J. Swartzendruber, P.A. Beck

The Au-Fe System

Bulletin of Alloy Phase Diagrams 5 (1984) 592-601

\section{Rätzke et al., 1999:}

K. Rätzke, M.J. Hall, D.B. Jardine, W.C. Shih, R.E. Somekh, A.L. Greer

Evolution of microstructure and magnetoresistance in $\mathrm{Co} / \mathrm{Cu}$ multilayers...

Journal of Magnetism and Magnetic Materials 204 (1999) 61-67

\section{Samwer et al., 1988:}

K. Samwer, H. Schroder, K. Pampus

Early Stage of the Solid State Amorphization of Crystalline Layers

Materials Science and Engeneering 97 (1988) 63-69

\section{Schleiwies, 1998:}

J. Schleiwies

Mikrocharakterisierung von nanostrukturierten $\mathrm{Cu} / \mathrm{Nb}$-Multilagen

Diplomarbeit, Universität Göttingen, 1998

\section{Schmahl et al., 1996:}

G. Schmahl, D. Rudolph, B. Niemann, P. Guttmann, J. Thieme, G. Schneider

Röntgenmikroskopie

Naturwissenschaften 83 (1996) 61-70

\section{Servi et al., 1966:}

I.S. Servi, D. Turnbull

Thermodynamics and Kinetics of Precipitation in the Copper-Cobalt System

Acta Metallurgica 14 (1966) 161-169

\section{Shi et al., 1998:}

H.T. Shi, D. Lederman

Surface smoothing and crystalline reorientation in thin cobalt films

Physical Review B 58 (1998) 1778-1781 
Shiftlet et al., 1981:

G.J. Shiftlet, Y.W. Lee, H.I. Aaronson, K.C. Russell

A Re-Assessment of the Comparison of Classical Homogenous Nucleation Theory

Scripta Metallurgica 15 (1981) 719-722

\section{Smithells:}

E. A. Brandes, Smithells Metals Reference Book

Butterworth, 1983

\section{Spiller, 1987:}

E. Spiller, Physics, Fabrication, and Applications of Multilayered Structures

Plenum, New York, 1987

Sridhar et al., 1997a:

N. Sridhar, J.M. Rickman, D.J. Srolovitz

Microstructural Stability of Stressed Lamellar and Fiber Composites

Acta Metallurgica 45 (1997) 2715-2733

Sridhar et al., 1997b:

N. Sridhar, J.M. Rickman, D.J. Srolovitz

Multilayer film stability

Journal of Applied Physics 82 (1997) 4852-4859

Störmer, 1998:

M. Störmer

Struktur, Mikrostruktur und innere Spannungen laserdep. met. Legierungen

Dissertation, Universität Göttingen, 1998

Streitz et al., 1994:

F.H. Streitz, R.C. Cammarata, K. Sieradzki

Surface-stress effects on elastic poperties. II. Metallic multilayers

Physical Review B 49 (1994) 10707-716

\section{Swartzendruber, 1984:}

\section{L.J. Swartzendruber}

Thermodynamics of The Ag-Fe System

Bulletin of Alloy Phase Diagrams 5 (1984) 560-564

\section{Troche, 1996:}

P. Troche

Gestaltinstabilität von Niobschichten in $\mathrm{Nb} / \mathrm{Cu}-\mathrm{Multilagenpaketen}$

Diplomarbeit, Universität Göttingen, 1996

Troche et al., 1999:

P. Troche, J. Hoffmann, F. Hartung, H.C. Freyhardt, D. Rudolph

Thermally Driven Shape Instabilities of $\mathrm{Nb} / \mathrm{Cu}$ Multilayer Structures

Thin Solid Films 353 (1999) 33-39 
Troche et al., 2000:

P. Troche, J. Hoffmann, C. Herweg, Ch. Lang, H.C. Freyhardt, D. Rudolph

Thermally Driven Shape Instability of Multilayer Structures

MRS Symposium Proceedings Vol. 581 (2000) 635-640

Utigard, 1993:

T. Utigard

Surface and Interfacial Energies of Metals

Zeitung für Metallkunde 84 (1993) 792-795

van der Merwe, 1972:

J.H. van der Merwe

Structure of Epitaxial Crystal Interfaces

Surface Science 31 (1972) 198-228

van Suchtelen, 1977:

J. van Suchtelen

Coarsening of Eutectic Structures During and After Unidirectional Growth

Journal of Crystal Growth 43 (1977) 28-46

Windischmann, 1987:

H. Windischmann

An Intrinsic Stress Scaling Law for Polycrystalline Thin Films

Journal of Applied Physics 62 (1987) 1800-1807

Windischmann, 1992:

Windischmann

Intrinsic Stress in Sputter-Deposited Thin Films

Critical Rev. in Solid State and Material Science 17 (1992) 547-596 


\section{Danksagung}

Herrn Prof. Dr. H.C. Freyhardt danke ich für die Anregung und Förderung dieser Arbeit. Vor allem aber danke ich für viele Impulse und Ideen zu diesem Projekt und eine sehr hilfreiche Versorgung mit aktueller Literatur.

Herrn Dr. Jörg Hoffmann gilt mein ganz besonderer Dank. Er stand mir bei zahlreichen Problemen, die sich bei der Bearbeitung dieses Themas ergaben, stets mit vielen Ideen und Lösungen zur Seite. Vor allem die ergiebigen Diskussionen in freundschaftlicher Runde in Haus D machten die letzten Jahre zu einer schönen Zeit.

Frau Dr. Christine Borchers danke ich dafür, dass sie die wunderbaren hochauflösenden TEM-Bilder von meinen Proben gemacht und mir mit viel Geduld die Energien von Grenzflächen näher gebracht hat.

Bei den Herren Christian Lang und Jörg Schleiwies bedanke ich mich ganz herzlich für die gute Zusammenarbeit im Bereich der Transmissionselektronenmikroskopie. Sie fanden immer Zeit, wenn ich mal wieder einen Blick in eine meiner Proben werfen wollte.

Bedanken möchte ich mich auch bei Herrn Dr. D. Rudolph für die röntgenmikroskopischen Bilder und seine stete Hilfsbereitschaft.

Frau Katrin Gehrke, Herrn Lutz Kirchhoff, Herrn Dieter Plischke und der ganzen Crew der Werkstatt danke ich für die Unterstützung bei den technischen Problemen.

Allen Mitgliedern der Arbeitsgruppe, besonders aber Frau Karola Thiele und Herrn Carsten Herweg, danke ich für die angenehme Zusammenarbeit und für viele schöne Stunden.

Meinem Vater danke ich für die sprachliche Korrektur meiner Arbeit. Frau Sonja Dahmen danke ich ebenfalls für sehr hilfreiche Korrekturen und eine schöne und lebendige Zeit in Göttingen.

Meinen Eltern Danke ich am meisten, insbesondere für den Rückhalt, den sie mir bei allen Problemen gewährten, und die Förderung meines Studiums sowie meiner wissenschaftlichen Laufbahn. Eure Hilfe hat mir vieles wesentlich erleichtert. 


\section{$\underline{\text { Lebenslauf }}$}

Am 18. Dezember 1971 wurde ich als erstes Kind des Studiendirektors Johannes Troche und seiner Frau, der Lehrerin Cläre Troche geb. Krumbach in Köln geboren.

Von 1978 bis 1982 besuchte ich die katholische Grundschule in Odenthal. Von dort wechselte ich auf das städtische Gymnasium im Schulzentrum Schlebusch in Leverkusen. Diese Schule besuchte ich bis 1991. Mein Abitur legte ich in den Leistungsfächern Physik und Mathematik, sowie den Grundkursfächern Englisch und Erkunde ab.

Im Herbst 1991 begann ich an der Georg August Universität in Göttingen das Physikstudium. Dort legte ich zum Ende des Sommersemesters 1993 mein Vordiplom ab. Im Wintersemester 1993 begann ich zudem das Studium der Betriebswirtschaftslehre. In diesem Fach legte ich das Vordiplom am Ende des Sommersemesters $1995 \mathrm{ab}$. Am 1. November 1996 erwarb ich am Institut für Materialphysik in der Arbeitsgruppe von Prof. Dr. H.C. Freyhardt nach einer Dauer von 18 Monaten das Diplom in Physik.

Seit November 1996 studiere ich im Aufbaustudiengang Physik mit dem Ziel der Promotion. 